\title{
GLOBAL STABILITY AND DECAY FOR THE CLASSICAL STEFAN PROBLEM
}

\author{
MAHIR HADŽIĆ AND STEVE SHKOLLER \\ Accepted for publication in Communications on Pure and Applied Mathematics \\ Department of Mathematics, King's College London, Strand, London, WC2R 2LS, UK \\ Mathematical Institute, University of Oxford, Andrew Wiles Building, Radcliffe \\ Observatory Quarter, Woodstock Road, Oxford, OX2 6GG, UK
}

\begin{abstract}
The classical one-phase Stefan problem describes the temperature distribution in a homogeneous medium undergoing a phase transition, such as ice melting to water. This is accomplished by solving the heat equation on a time-dependent domain whose boundary is transported by the normal derivative of the temperature along the evolving and a priori unknown free-boundary. We establish a global-in-time stability result for nearly spherical geometries and small temperatures, using a novel hybrid methodology, which combines energy estimates, decay estimates, and Hopf-type inequalities.
\end{abstract}

\section{CONTENTS}

1. Introduction

1.1. The problem formulation

1.2. The reference domain $\Omega$ and the dimension

1.3. Notation

1.4. Fixing the domain

1.5. A brief history of prior results on the Stefan problem 9

1.6. Methodology and outline of the paper 10

2. Bootstrap assumptions and a priori bounds 13

2.1. Poincaré-type inequality 14

2.2. A priori bounds on $h \quad 15$

2.3. Differentiation rules for $A \quad 18$

2.4. Estimates for $\nabla \Psi-\operatorname{Id}$ and $A-\mathrm{Id}$

2.5. High-order derivatives of $q \quad 18$

2.6. Lower bound on $\chi(t) \quad 24$

3. Energy identity and the higher-order energy estimate 26

3.1. The energy identity 26

3.2. Energy estimates 27

4. Existence for all time $t \geq 0$ and nonlinear stability 32

4.1. Structure of the proof 32

E-mail address: mahir.hadzic@kcl.ac.uk, shkoller@maths.ox.ac.uk.

1991 Mathematics Subject Classification. 35R35, 35B65 , 35K05, 80A22.

Key words and phrases. free-boundary problems, Stefan problem, regularity, stability, global existence. 
4.2. Boundedness of $E_{\beta}$

4.3. Pointwise positivity of $\partial_{N} q_{t}$ at time $T_{K}=\bar{C} \ln K$

4.4. Proof of Theorem 1.2

5. The $d$-dimensional case on general near-spherical domains 40

Appendix A. Proof of Proposition 3.1 42

A.1. Identities of the first type $\quad 42$

A.2. Identities of the second type 4

Appendix B. Proof of the inequality (75)

\begin{tabular}{ll|} 
Acknowledgements & 49 \\
\hline
\end{tabular}

References

\section{INTRODUCTION}

1.1. The problem formulation. We consider the problem of global existence and asymptotic stability of classical solutions to the classical Stefan problem describing the evolving free-boundary between the liquid and solid phases. The temperature of the liquid $p(t, x)$ and the a priori unknown moving phase boundary $\Gamma(t)$ must satisfy the following system of equations:

$$
\begin{aligned}
& p_{t}-\Delta p=0 \quad \text { in } \Omega(t) \text {; } \\
& \mathscr{V}(\Gamma(t))=-\partial_{n} p \quad \text { on } \Gamma(t) \text {; } \\
& p=0 \quad \text { on } \Gamma(t) \text {; } \\
& p(0, \cdot)=p_{0}, \Omega(0)=\Omega_{0} .
\end{aligned}
$$

For each instant of time $t \in[0, T], \Omega(t)$ is a time-dependent open subset of $\mathbb{R}^{d}$ with $d \geq 2$, and $\Gamma(t):=\partial \Omega(t)$ denotes the moving, time-dependent free-boundary.

The heat equation $1 \mathrm{a}$ models thermal diffusion in the bulk $\Omega(t)$ with thermal diffusivity set to 1 . The boundary transport equation (1b) states that each point on the moving boundary is transported with normal velocity equal to $-\partial_{n} p=-\nabla p \cdot n$, the normal derivative of $p$ on $\Gamma(t)$. Here $n$ denotes the outward pointing unit normal to $\Gamma(t)$, and $\mathscr{V}(\Gamma(t))$ denotes the speed or the normal velocity of the hypersurface $\Gamma(t)$. The homogeneous Dirichlet boundary condition (1c) is termed the classical Stefan condition and problem (1) is called the classical Stefan problem. It implies that the freezing of the liquid occurs at a constant temperature $p=0$. Finally, we must specify the initial temperature distribution $p_{0}: \Omega_{0} \rightarrow \mathbb{R}$, as well as the initial geometry $\Omega_{0}$. Because the liquid phase $\Omega(t)$ is characterized by the set $\left\{x \in \mathbb{R}^{d}: p(x, t)>0\right\}$, we shall consider initial data $p_{0}>0$ in $\Omega_{0}$. Problem (1) belongs to the category of free boundary problems which are of parabolichyperbolic type. Thanks to $1 \mathrm{a}$, , the parabolic Hopf lemma implies that $\partial_{n} p(t)<0$ on $\Gamma(t)$ for $t>0$, so we impose the non-degeneracy condition or so-called Taylor sign condition 1

$$
-\partial_{n} p_{0} \geq \lambda>0 \quad \text { on } \Gamma(0)
$$

on our initial temperature distribution. Under the above assumptions, we proved in Hadžić \& Shkoller [29] that [1] is indeed well-posed.

\footnotetext{
${ }^{1}$ This type of stability condition dates back to the early work of Lord Rayleigh [46] and Taylor [48] in fluid mechanics, and appears as a necessary well-posedness condition on the initial data in many free-boundary problems wherein the effects of surface tension are ignored; examples include the Hele-Shaw cell, the water waves equations [50], and the full Euler equations in both incompressible [15] and compressible form [18, 17].
} 
1.2. The reference domain $\Omega$ and the dimension. For our reference domain, we choose the unit ball in $\mathbb{R}^{2}$ given by

$$
\Omega=B(0,1):=\left\{x \in \mathbb{R}^{2}:|x|<1\right\},
$$

with boundary $\Gamma=\mathbb{S}^{1}:=\left\{x \in \mathbb{R}^{2}:|x|=1\right\}$. We shall consider initial domains $\Omega_{0}$ whose boundary $\Gamma_{0}$ is a graph over the reference boundary $\Gamma$. In order to simplify our presentation, we consider evolving domains $\Omega(t)$ in $\mathbb{R}^{2}$, but as we shall explain in Section 5 , our methodology works equally well in any dimension $d \geq 2$.

Our choice of the reference domain $\Omega$ follows from two considerations. First, we need employ only one global coordinate system near the boundary $\Gamma$, rather than a collection of local coordinate charts that a more general domain would necessitate, and the use of one coordinate system greatly simplifies the presentation of our energy identities, that provide very natural estimates for the second-fundamental form of the evolving free-boundary $\Gamma(t)$. Second, we shall need quantitative Hopf-type inequalities in order to bound the term defined in (2) from below, and such estimates are available in a particularly satisfying form in the case of the nearly spherical domains, thanks to the explicit construction of comparison functions in ODDSON [41].

1.3. Notation. For any $s \geq 0$ and given functions $f: \Omega \rightarrow \mathbb{R}, \varphi: \Gamma \rightarrow \mathbb{R}$ we set

$$
\|f\|_{s}:=\|f\|_{H^{s}(\Omega)} \text { and }|\varphi|_{s}:=\|\varphi\|_{H^{s}(\Gamma)} .
$$

$H^{s}(\Omega)^{\prime}$ shall denote the dual space of $H^{s}(\Omega)$, while on the boundary, $H^{s}(\Gamma)^{\prime}=H^{-s}(\Gamma)$. If $i=1,2$ then $f_{, i}:=\partial_{x^{i}} f$ is the partial derivative of $f$ with respect to $x^{i}$. Similarly, $f_{, i j}:=$ $\partial_{x^{i}} \partial_{x^{j}} f$, etc. For time-differentiation, $f_{t}:=\partial_{t} f$. Furthermore, for a function $f(t, x)$, we shall often write $f(t)$ for $f(t, \cdot)$, and $f(0)$ to mean $f(0, x)$. We use $\bar{\partial}:=\tau \cdot \nabla$ to denote the tangential derivative, so that

$$
\bar{\partial} f:=\partial_{\theta} f, \quad \bar{\partial}^{k} f:=\partial_{\theta}^{k} f,
$$

where $\theta \in[0,2 \pi)$ denotes the angular component in polar coordinates. The Greek letter $\alpha$ will often be reserved for multi-indices $\alpha=\left(\alpha_{1}, \alpha_{2}\right)$, with $\partial^{\alpha}:=\partial_{x_{1}}^{\alpha_{1}} \partial_{x_{2}}^{\alpha_{2}}$ and $|\alpha|=$ $\alpha_{1}+\alpha_{2}$. The identity map on $\Omega$ is denoted by $e(x)=x$, while the identity matrix is denoted by Id. We use $C$ to denote a universal (or generic) constant that may change from inequality to inequality. We write $X \lesssim Y$ to denote $X \leq C Y$. We use the notation $P(\cdot)$ to denote generic real polynomial function of its argument(s) with positive coefficients. The Einstein summation convention is employed, indicating summation over repeated indices. The $L^{2}$ inner product on $\Omega$ is denoted by $(\cdot, \cdot)_{L^{2}}$.

1.4. Fixing the domain. We transform the Stefan problem (1), set on the moving domain $\Omega(t)$, to an equivalent problem on the fixed domain $\Omega$. For many problems in fluid dynamics, the Lagrangian flow map of the fluid velocity provides a natural family of diffeomorphisms which can be used to fix the domain, but for the classical Stefan problem, we use instead (in the parlance of fluid dynamics) the so-called Arbitrary Lagrangian-Eulerian (ALE) family of diffeomorphisms; these ALE maps interpolate between the Lagrangian and Eulerian representations of the equations. For this problem, we choose a simple type of ALE map, consisting of harmonic coordinates, also known as the harmonic gauge.

1.4.1. The diffeormorphism $\Psi(t)$. We represent our moving domain $\Omega(t)$ as the image of a time-dependent family of diffeomorphisms $\Psi(t): \Omega \rightarrow \Omega(t)$. In order to define these 
diffeomorphisms, we let $h(t, \cdot): \Gamma \rightarrow \mathbb{R}$ denote the signed height function whose graph (over $\Gamma$ ) is the set $\Gamma(t)$. For $\xi \in \Gamma=\mathbb{S}^{1}$, we define the map

$$
\Psi(t, \xi)=(1+h(t, \xi)) \xi=R(t, \xi) \xi
$$

which is a diffeomorphism of $\Gamma$ onto $\Gamma(t)$ as long as $h(t)$ remains a graph. The outwardpointing unit normal vector $n(t, \cdot)$ to the moving surface $\Gamma(t)$ is defined by

$$
(n \circ \Psi)(t, \xi)=(R \xi) \frac{\perp}{\theta} /\left|(R \xi) \frac{\perp}{\theta}\right| .
$$

We shall henceforth drop the explicit composition with the diffeomorphism $\Psi$, and simply write

$$
n(t, \xi)=(R \xi)_{\theta}^{\perp} /\left|(R \xi) \frac{\perp}{\theta}\right|
$$

for the unit normal to the moving boundary at the point $\Psi(t, \xi) \in \Gamma(t)$.

Introducing the unit normal and tangent vectors to the reference surface $\Gamma$ as (3)

$$
N:=\xi, \quad \tau:=\xi_{\theta} \quad \text { or equivalently } \quad N(\theta)=(\cos \theta, \sin \theta), \quad \tau(\theta)=(-\sin \theta, \cos \theta),
$$

we write the unit normal to $\Gamma(t)$ as

$$
n(t, \xi)=\frac{R N-h_{\theta} \tau}{\sqrt{R^{2}+R_{\theta}^{2}}} .
$$

The evolution of $h(t)$ is then given by

$$
h_{t}=v \cdot N(\theta)-\frac{h_{\theta}}{R} v \cdot \tau(\theta) .
$$

Assuming that the signed height function $h(t, \cdot)$ is sufficiently regular and remains a graph, we can define a diffeomorphism $\Psi: \Omega \rightarrow \Omega(t)$ as the elliptic extension of the boundary diffeomorphism $\xi \mapsto(1+h(\xi, t)) \xi$, by solving the following Dirichlet problem

$$
\begin{aligned}
\Delta \Psi & =0 \text { in } \Omega, \\
\Psi(t, \xi) & =R(t, \xi) \xi \quad \xi \in \Gamma .
\end{aligned}
$$

Since the identity map $e: \Omega \rightarrow \Omega$ is harmonic in $\Omega$ and $\Psi-e=h \xi$ on $\Gamma$, standard elliptic regularity theory for solutions to $(6)$ shows that

$$
\|\Psi-e\|_{H^{s}(\Omega)} \leq C\|h\|_{H^{s-0.5}(\Gamma)}, s>0.5,
$$

so that for $h(t)$ sufficiently small and $s$ large enough, the Sobolev embedding theorem shows that $\nabla \Psi(t)$ is close to the identity matrix Id, and by the inverse function theorem, each $\Psi(t)$ is a diffeomorphism.

1.4.2. The temperature and velocity variables on the fixed domain $\Omega$. First we introduce the velocity variable $u=-\nabla p$ in $\Omega(t)$. Next, we introduce the following new variables set on the fixed domain $\Omega$ :

$$
\begin{aligned}
q & =p \circ \Psi & & \text { (temperature), } \\
v & =u \circ \Psi & & \text { (velocity), } \\
w & =\Psi_{t} & & \text { (extension of boundary velocity vector), } \\
A & =[D \Psi]^{-1} & & \text { (inverse of the deformation tensor), } \\
J & =\operatorname{det} D \Psi & & \text { (Jacobian determinant), } \\
a & =J A & & \text { (cofactor matrix of the deformation tensor). }
\end{aligned}
$$


The relation $u=-\nabla p$ is then written as $v^{i}+A_{i}^{k} q, k=0$ for $i=1,2$. By the chain rule,

$$
q_{t}=p_{t} \circ \Psi+(\nabla p \circ \Psi) \cdot \Psi_{t}=p_{t} \circ \Psi-v \cdot w,
$$

and

$$
\Delta p \circ \Psi=\Delta \Psi q:=A_{i}^{j}\left(A_{i}^{k} q, k\right), j .
$$

Letting $\tilde{n}=J^{-1}\left(R^{2}+R_{\theta}^{2}\right)^{\frac{1}{2}} n$, we see that

$$
\tilde{n}_{i}(t, x)=A_{i}^{k}(t, x) N_{k}(x)
$$

and equation (5) can be written as $h_{t}=v \cdot \tilde{n} / R_{J}$, where $R_{J}=R J^{-1}$. Note that $R_{J}=R J^{-1}=$ $(1+h) J^{-1}$ is very close to 1 .

1.4.3. The classical Stefan problem set on the fixed domain $\Omega$. The classical Stefan problem on the fixed domain $\Omega$ is written as

$$
\begin{aligned}
q_{t}-A_{i}^{j}\left(A_{i}^{k} q, k\right), j & =-v \cdot \Psi_{t} & & \text { in }(0, T] \times \Omega, \\
v^{i}+A_{i}^{k} q_{, k} & =0 & & \text { in }[0, T] \times \Omega, \\
q & =0 & & \text { on }[0, T] \times \Gamma, \\
h_{t} & =v \cdot N-(1+h)^{-1} h_{\theta} v \cdot \tau & & \text { on }(0, T] \times \Gamma, \\
\Delta \Psi & =0 & & \text { on }[0, T] \times \Omega, \\
\Psi & =(1+h) N & & \text { on }[0, T] \times \Gamma, \\
q & =q_{0}>0 & & \text { on }\{t=0\} \times \Omega, \\
h & =h_{0} & & \text { on }\{t=0\} \times \Gamma,
\end{aligned}
$$

where the initial boundary $\partial \Omega_{0}$ is given as a graph over $\Omega$ with the initial height function $h_{0}$, i.e. $\partial \Omega_{0}=\left\{x \in \mathbb{R}^{2}, x=\left(1+h_{0}(\xi)\right) \xi, \xi \in \mathbb{S}^{1}\right\}$. Note that $\Phi=\Psi(0): \Omega \rightarrow \Omega_{0}$ is a near identity transformation, mapping the reference domain $\Omega$ onto the initial domain $\Omega_{0}$. The initial temperature function $q_{0}$ equals $p_{0} \circ \Phi$. Problem 9 is a reformulation of the problem (1).

Henceforth, without loss of generality, we shall assume that the initial domain $\Omega_{0}$ is the unit ball $B_{1}(0)$ or in other words $h_{0}=0$. In this case, we set $\Phi=e$, where $e: \Omega \rightarrow \Omega$ is the identity map, and $\left.\Psi(t)\right|_{t=0}=e$. In Section 5, we will explain the minor modification required when $h_{0} \neq 0$, as well as the case that the dimension $d=3$.

Observe that the boundary condition $9 \mathrm{~d}$ implies that

$$
\Psi_{t} \cdot n(t)=v \cdot n(t) \text { on }[0, T] \times \Gamma \text { so that } \Psi(t)(\Gamma)=\Gamma(t) .
$$

1.4.4. The energy and dissipation functions. Near $\Gamma=\partial \Omega$, it is convenient to use tangential derivatives $\bar{\partial}:=\partial_{\theta}$ with $\theta$ denoting the polar angle, while near the origin, Cartesian partial derivatives $\partial_{x_{i}}$ are natural. For this reason, we introduce a non-negative $C^{\infty}$ cut-off function $\mu: \bar{\Omega} \rightarrow \mathbb{R}_{+}$with the property

$$
\mu(x) \equiv 0 \text { if }|x| \leq 1 / 2 ; \quad \mu(x) \equiv 1 \quad \text { if } 3 / 4 \leq|x| \leq 1 .
$$


Definition 1.1 (Higher-order norms). The following high-order energy and dissipation functionals are fundamental to our analysis:

$$
\begin{aligned}
\mathscr{E}(t) & =\mathscr{E}(q, h)(t) \\
& :=\frac{1}{2} \sum_{a+2 b \leq 5}\left\|\mu^{1 / 2} \bar{\partial}^{a} \partial_{t}^{b} v\right\|_{L_{x}^{2}}^{2}+\frac{1}{2} \sum_{b=0}^{2}\left|\left(-\partial_{N} q\right)^{1 / 2} R J^{-1} \bar{\partial}^{6-2 b} \partial_{t}^{b} h\right|_{L_{x}^{2}}^{2}+\frac{1}{2} \sum_{a+2 b \leq 6}\left\|\mu^{1 / 2}\left(\bar{\partial}^{a} \partial_{t}^{b} q+\bar{\partial}^{a} \partial_{t}^{b} \Psi \cdot v\right)\right\|_{L_{x}^{2}}^{2} \\
& +\sum_{|\vec{\alpha}|+2 b \leq 5}\left\|(1-\mu)^{1 / 2} \partial_{\vec{\alpha}} \partial_{t}^{b} v\right\|_{L_{x}^{2}}^{2}+\frac{1}{2} \sum_{|\vec{\alpha}|+2 b \leq 6}\left\|(1-\mu)^{1 / 2}\left(\partial_{\vec{\alpha}} \partial_{t}^{b} q+\partial_{\vec{\alpha}} \partial_{t}^{b} \Psi \cdot v\right)\right\|_{L_{x}^{2}}^{2}
\end{aligned}
$$

and

$$
\begin{aligned}
\mathscr{D}(t) & =\mathscr{D}(q, h)(t) \\
& :=\sum_{a+2 b \leq 6}\left\|\mu^{1 / 2} \bar{\partial}^{a} \partial_{t}^{b} v\right\|_{L_{x}^{2}}^{2}+\sum_{b=0}^{2}\left|\left(-\partial_{N} q\right)^{1 / 2} R J^{-1} \bar{\partial}^{5-2 b} \partial_{t}^{b} h_{t}\right|_{L_{x}^{2}}^{2}+\sum_{a+2 b \leq 5}\left\|\mu^{1 / 2}\left(\bar{\partial}^{a} \partial_{t}^{b} q_{t}+\bar{\partial}^{a} \partial_{t}^{b} \Psi_{t} \cdot v\right)\right\|_{L_{x}^{2}}^{2} \\
& +\sum_{|\vec{\alpha}|+2 b \leq 6}\left\|(1-\mu)^{1 / 2} \partial_{\vec{\alpha}} \partial_{t}^{b} v\right\|_{L_{x}^{2}}^{2}+\sum_{|\vec{\alpha}|+2 b \leq 5}\left\|(1-\mu)^{1 / 2}\left(\partial_{\vec{\alpha}} \partial_{t}^{b} q_{t}+\partial_{\vec{\alpha}} \partial_{t}^{b} \Psi_{t} \cdot v\right)\right\|_{L_{x}^{2}}^{2} .
\end{aligned}
$$

Note that the boundary norms of the height function are weighted by $\sqrt{-\partial_{N} q}$. We thus introduce the time-dependent function

$$
\chi(t):=\inf _{x \in \Gamma}\left(-\partial_{N} q\right)(t, x)>0,
$$

which will be used to track the weighted behavior of $h$. We will show that $\mathscr{E}$ is indeed equivalent to

$$
\sum_{l=0}^{3}\left\|\partial_{t}^{l} q\right\|_{H^{6-2 l}(\Omega)}^{2}+\chi(t) \sum_{l=0}^{3}\left|\partial_{t}^{l} h\right|_{H^{6-2 l}(\Gamma)}^{2},
$$

and that $\mathscr{D}$ is equivalent to

$$
\|q\|_{H^{6.5}(\Omega)}^{2}+\sum_{l=0}^{2}\left\|\partial_{t}^{l} q_{t}\right\|_{H^{5-2 l}(\Omega)}^{2}+\chi(t) \sum_{l=0}^{2}\left|\partial_{t}^{l+1} h\right|_{H^{5-2 l}(\Gamma)}^{2} .
$$

The elliptic operator in the parabolic equation (9a) for $q$ has coefficients that depend on $A=[D \Psi]^{-1}$, which in turn depend on $h$; hence, the regularity of $q$ is limited (and, in fact, determined) by the regularity of $h$ on the boundary $\Gamma$. Since the regularity of $h$ is given by norms which are weighted by the factor $\chi(t)$, a naive application of elliptic estimates would thus lead to the crude bound

$$
\left\|\partial_{t}^{l} q\right\|_{6.5-2 l}^{2} \lesssim \frac{\mathscr{D}}{\chi(t)}
$$

which could a priori grow in time. However, by using the fact that lower-order norms of $q$ have exponential decay (in time), estimate (13) can be improved to yield

$$
\|q\|_{6.5}^{2}+\left\|\partial_{t}^{l} q_{t}\right\|_{5-2 l}^{2} \lesssim e^{-\gamma t} \mathscr{E}+\mathscr{D}, \quad l=0, \ldots, 2
$$

for some positive constant $\gamma>0$. This is one of the essential ingredients of our analysis, as (14) will be used to control error terms arising from higher-order energy estimates in Section 3 
In order to capture the exponential decay of the temperature $q$, we introduce the lowerorder decay norms:

$E_{\beta}(t):=e^{\beta t}\left(\sum_{b=0}^{2}\left\|\partial_{t}^{b} q(t)\right\|_{H^{4-2 b}(\Omega)}^{2}+\sum_{b=0}^{1}\left\|\partial_{t}^{b} v\right\|_{H^{3-2 b}(\Omega)}^{2}\right), \quad D(t):=\sum_{b=0}^{2}\left\|\partial_{t}^{b} q(t)\right\|_{H^{5-2 b}(\Omega)}^{2}$,

with the constant $\beta$ denoting a positive real number given by

$$
\beta:=2 \lambda_{1}-\eta
$$

where $\lambda_{1}$ is the smallest eigenvalue of the Dirichlet-Laplacian on $\Omega=B_{1}(0)$ and $\eta$ is a small positive constant related to the size of the initial data, which will be made precise below. Note that the smallness of $E_{\beta}$ in particular implies an exponential decay (in time) estimate for the $H^{4}$-norm of the temperature $q(t)$.

1.4.5. Taylor sign condition or non-degeneracy condition on $q_{0}$. With respect to $q_{0}=$ $p_{0} \circ \Phi$, condition (2) becomes $\inf _{x \in \Gamma}\left[-\partial_{N} q_{0}(x)\right] \geq \lambda>0$ on $\Gamma$. For initial temperature distributions that are not necessarily strictly positive in $\Omega$, this condition was shown to be necessary for local well-posedness for (11) (see [29, 40, 42]). On the other hand, if we require strict positivity of our initial temperature function,

$$
q_{0}>0 \quad \text { in } \Omega,
$$

then the parabolic Hopf lemma (see, for example, [21]) guarantees that $-\partial_{N} q(t, x)>0$ for $0<t<T$ on some a priori (possibly small) time interval, which, in turn, shows that $\mathscr{E}$ and $\mathscr{D}$ are norms for $t>0$, but uniformity may be lost as $t \rightarrow 0$. To ensure a uniform lower-bound for $-\partial_{N} q(t)$ as $t \rightarrow 0$, we impose the Taylor sign condition with the following lower-bound:

$$
-\partial_{N} q_{0} \geq C \int_{\Omega} q_{0} \varphi_{1} d x
$$

Here, $\varphi_{1}$ is the positive first eigenfunction of the Dirichlet-Laplacian, and $C>0$ denotes a universal constant. The uniform lower-bound in (18) thus ensures that our solutions are continuous in time; moreover, (18) allows us to establish a time-dependent optimal lowerbound for the quantity $\chi(t)=\inf _{x \in \Gamma}\left(-\partial_{N} q\right)(t, x)>0$ for all time $t \geq 0$, which will be crucial for our analysis.

1.4.6. Compatibility conditions. The definition of our higher-order energy function $\mathscr{E}$, restricted to time $t=0$, requires an explanation of the time-derivates of $q$ and $h$ evaluated at $t=0$. Specifically, the values $\left.q_{t}\right|_{t=0},\left.q_{t t}\right|_{t=0},\left.h_{t}\right|_{t=0}$ and $\left.h_{t t}\right|_{t=0}$ are defined via spacederivatives using equations $9 \mathrm{a}$ and $9 \mathrm{~d}$. To ensure that the solution is continuously differentiable in time at $t=0$ we must impose compatibility conditions on the initial data (such conditions are, of course, only necessary for regular initial data). By restricting the equation (9a) to the boundary at time $t=0$ and using the fact that $q_{t}(0)=0$ on $\Gamma$ and that $\left.A_{i}^{k}\right|_{t=0}=\delta_{i}^{k}$, where $\delta_{i}^{k}$ denotes the Kronecker delta which equals 1 if $k=i$ and 0 otherwise, we obtain the first-order compatibility condition

$$
\Delta q_{0}=\left(\partial_{N} q_{0}\right)^{2} \text { on } \Gamma \text {. }
$$

\footnotetext{
${ }^{2}$ Condition 17 is natural, since it determines the phase: $\Omega(t)=\{q(t)>0\}$.

${ }^{3}$ When $h_{0} \neq 0$, the unit normal to the initial surface $\Gamma_{0}$ is given by $N=\frac{\left(1+h_{0}\right) \xi-\partial_{\theta} h_{0} \tau}{\sqrt{\left(1+h_{0}\right)^{2}+\partial_{\theta} h_{0}^{2}}}$ where $\xi=(\cos \theta, \sin \theta)$ and $\tau=(-\sin \theta, \cos \theta)$.
} 
Upon differentiating $9 \mathrm{a}$ with respect to time, and then restricting to $\Gamma$ at $t=0$ and using (19), we arrive at the second-order compatibility condition

$$
\Delta^{2} q_{0}=\Delta\left|\partial_{N} q_{0}\right|^{2}+2 \partial_{N}\left(\Delta q_{0}-\left|\partial_{N} q_{0}\right|^{2}\right) \partial_{N} q_{0}-2\left|\partial_{N N} q_{0}\right|^{2} \quad \text { on } \Gamma,
$$

where we have used that $h_{t}(t, \theta)=v \cdot\left[N(\theta)-\tau(\theta) h_{\theta}(1+h)^{-1}\right]$.

We note that our functional framework only requires specification of two higher-order compatibility conditions (the condition $q_{0}=0$ on $\Gamma$ being the zeroth-order condition).

1.4.7. Main result. Our main result is a global-in-time stability theorem for solutions of the classical Stefan problem for surfaces which are nearly spherical and for temperature fields close to zero. The notion of "near" is measured by our energy norms as well as the dimensionless quantity

$$
K:=\frac{\left\|q_{0}\right\|_{4}}{\left\|q_{0}\right\|_{0}}
$$

as expressed in the following

Theorem 1.2. Let $\left(q_{0}, h_{0}\right)$ satisfy the Taylor sign condition (18), the strict positivity assumption (17), and the compatibility conditions (19), (20). Let $K$ be defined as in (21). Then there exists an $\varepsilon_{0}>0$ and a monotonically increasing function $F:(1, \infty) \rightarrow \mathbb{R}_{+}$, such that if

$$
\mathscr{E}\left(q_{0}, h_{0}\right)<\frac{\varepsilon_{0}^{2}}{F(K)},
$$

then there exist unique solutions $(q, h)$ to problem (9) satisfying

$$
\sup _{0 \leq t \leq \infty} \mathscr{E}(q(t), h(t))<C \varepsilon_{0}^{2}
$$

for some universal constant $C>0$. Moreover, the temperature $q(t) \rightarrow 0$ as $t \rightarrow \infty$ with bound

$$
\|q\|_{H^{4}(\Omega)}^{2} \leq C e^{-\beta t},
$$

where $\beta=2 \lambda_{1}-O\left(\varepsilon_{0}\right)$ and $\lambda_{1}$ is the smallest eigenvalue of the Dirichlet-Laplacian on the unit disk. The moving boundary $\Gamma(t)$ settles asymptotically to some nearby steady surface $\bar{\Gamma}$ and we have the uniform-in-time estimate

$$
\sup _{0 \leq t<\infty}\left|h-h_{0}\right|_{4.5} \lesssim \sqrt{\varepsilon_{0}}
$$

Remark 1.3. The increasing function $F(K)$ given in (22) has an explicit form. For generic constants $\bar{C}, C>1$ chosen in Sections 3 and 4 below,

$$
F(K):=\max \left\{8 K^{2 C \bar{C} K^{2}}, \bar{C}^{10}(\ln K)^{10} K^{20 \bar{C} \lambda_{1}}\right\} .
$$

Remark 1.4. The use of the constant $K$ in our smallness assumption (22) allows us to determine a time $T=T_{K}$ when the dynamics of the Stefan problem become strongly dominated by the projection of $q$ onto the first eigenfunction $\varphi_{1}$ of the Dirichlet-Laplacian. Explicit knowledge of the $K$-dependence in the smallness assumption (22) permits the use of energy estimates to show that solutions exist in our energy space on the time-interval $\left[0, T_{K}\right]$. For $t \geq T_{K}$, certain error terms (that cannot be controlled by our energy and dissipation functions for large $t$ ) become sign-definite with a good sign. 
1.5. A brief history of prior results on the Stefan problem. There is a large amount of literature on the classical one-phase Stefan problem. For an overview we refer the reader to Friedman [23], MeIrmanov [40] and Visintin [49]. First, weak solutions were defined by KAMENOMOSTSKAYA [32], FRIEDMAn [22], and LADYZHENSKAYA, SOLONNIKOV \& URAL'CEVA [38]. For the one-phase problem studied herein, a variational formulation was introduced by FRIEDMAN \& KINDERLEHRER [24], wherein additional regularity results for the free surface were obtained. CAFARELLI [5] showed that in some space-time neighborhood of points $x_{0}$ on the free-boundary that have Lebesgue density, the boundary is $C^{1}$ in both space and time, and second derivatives of temperature are continuous up to the boundary. Under some regularity assumptions on the temperature, Lipschitz regularity of the free boundary was shown by CAFARELLI [6]. In related work, KINDERLEHRER \& NIRENBERG [35, 36] showed that the free boundary is analytic in space and of second Gevrey class in time, under the a priori assumption that the free boundary is $C^{1}$ with certain assumptions on the temperature function. In [7], CAFFARELLI $\&$ FRIEDMAN showed the continuity of the temperature in $d$ dimensions. As for the twophase classical Stefan problem, the continuity of the temperature in $d$ dimensions for weak solutions was shown by CAFFARELLI \& EVANS [8].

Since the Stefan problem satisfies a maximum principle, its analysis is ideally suited to another type of weak solution called the viscosity solution. Regularity of viscosity solutions for the two-phase Stefan problem was established by ATHANASOPOULOS, CAFFARELLI \& SALSA in a series of seminal papers [3, 4]. Existence of viscosity solutions for the one-phase problem was established by KIM [33], and for the two-phase problem by KIM \& POŽAR [34]. A local-in-time regularity result was established by CHOI \& KIM [11], where it was shown that initially Lipschitz free-boundaries become $C^{1}$ over a possibly smaller spatial region. For an exhaustive overview and introduction to the regularity theory of viscosity solutions we refer the reader to CAFFARELLI \& SALSA [9]. In [37], $\mathrm{KOCH}$ showed by the use of von Mises variables and harmonic analysis, that an priori $C^{1}$ free-boundary in the two-phase problem becomes smooth.

Local existence of classical solutions for the classical Stefan problem was established by Meirmanov (see [40] and references therein) and Hanzawa [30]. Meirmanov regularized the problem by adding artificial viscosity to $1 \mathrm{~b}$ and fixed the moving domain by switching to the so-called von Mises variables, obtaining solutions with less Sobolevregularity than the initial data. Similarly, Hanzawa used Nash-Moser iteration to construct a local-in-time solution, but again, with derivative loss. A local-in-time existence result for the one-phase multi-dimensional Stefan problem was proved by FroLOVA \& SolonNikov [26], using $L^{p}$-type Sobolev spaces. For the two-phase Stefan problem, a local-in-time existence result for classical solutions was established by PRÜSS, SAAL, \& SIMONETT [42] in the framework of $L^{p}$-maximal regularity theory.

In a related work, local existence for the two-dimensional two-phase Muskat problem (with varying viscosity and density) was proved by CóRDOBA, CóRDOBA \& GANCEDo [13] and in three dimensions in [14]. Their methods rely on a boundary-integral formulation for the Muskat problem, together with the Taylor sign condition. In a subsequent work [12], various global existence results were established. An overview can be found in [10].

As to the Stefan problem with surface tension (also known as the Stefan problem with Gibbs-Thomson correction), global weak solutions (without uniqueness) were given by Almgren \& Wang, Luckhaus, and Röger [2, 39, 47]. In Friedman \& ReITICH [25] the authors considered the Stefan problem with small surface tension, i.e. with 
$\sigma \ll 1$, whereby $(1 \mathrm{c})$ is replaced by $v=\sigma \kappa, \kappa$ denoting mean curvature of the boundary. Local existence of classical solutions was studied by RADKEVICH [45]; ESCHER, PRÜSS, \& SIMONETT [20] proved a local existence and uniqueness result for classical solutions under a smallness assumption on the initial height function close to the reference flat boundary. Global existence close to flat hyper-surfaces was proved by HADŽIĆ \& GUO in [28], and close to stationary spheres for the two-phase problem in HADŽIĆ [27] and PRÜSS, SIMONETT, \& ZACHER [43].

In order to understand the asymptotic behavior of the classical Stefan problem on external domains, QUIRós \& VÁZQUEZ [44] proved that on a complement of a given bounded domain $G$, with non-zero boundary conditions on the fixed boundary $\partial G$, the solution to the classical Stefan problem converges, in a suitable sense, to the corresponding solution of the Hele-Shaw problem and sharp global-in-time expansion rates for the expanding liquid blob are obtained. Moreover, the blob asymptotically has the geometry of a ball. Note that the non-zero boundary conditions act as an effective forcing which is absent from our problem and the techniques of [44] do not directly apply. Since the corresponding HeleShaw problem (in the absence of surface tension and forcing) is not a dynamic problem, possessing only time-independent solutions, we are not able to use the Hele-Shaw solution as a comparison problem for our problem.

A global stability result for the two-phase classical Stefan problem in a smooth functional framework was also established by MEIRMANOv [40] for a specific (and somewhat restrictive) perturbation of a flat interface, wherein the initial geometry is a strip with imposed Dirichlet temperature conditions on the fixed top and bottom boundaries, allowing for only one equilibrium solution. A global existence result for smooth solutions was given by DASKALOPOULOS \& LEE [19] under the log-concavity assumption on the initial temperature function, which in light of the level-set reformulation of the Stefan problem, requires convexity of the initial domain (a property that is preserved by the dynamics).

In [29], we established the local-in-time existence, uniqueness, and regularity for the classical Stefan problem in $L^{2}$ Sobolev spaces, without derivative loss, using the functional framework given by (11) and (12). This framework is natural, and relies on the geometric control of the free-boundary, analogous to that used in the analysis of the free-boundary incompressible Euler equations in COUTAND \& SHKOLLER [15, 16]; the second-fundamental form is controlled by a a natural coercive quadratic form, generated from the inner-product of the tangential derivative of the cofactor matrix $a$, and the tangential derivative of the velocity of the moving boundary, and yields control of the norm $\int_{\Gamma}\left(-\partial_{N} q(t)\right)\left|\bar{\partial}^{k} h\right|^{2} d x^{\prime}$ for any $k \geq 3$. The Hopf lemma ensures positivity of $-\partial_{N} q(t)$ and the Taylor sign condition on $q_{0}$ ensures a uniform lower-bound as $t \rightarrow 0$; on the other hand, $-\partial_{N} q(t) \rightarrow 0$ as $t \rightarrow \infty$, and so an optimal lower-bound for $\left(-\partial_{N} q(t)\right)$ for large $t$ is essential to establish a global existence and stability theory.

We remark that global stability of solutions in the presence of surface tension (see, for example, [28, 27, 43]) does not require the use of function framework with a decaying weight, such as $-\partial_{N} q(t)$. In this regard, the surface tension problem is simpler for two important reasons: first, the surface tension contributes a positive-definite energy-contribution that is uniform-in-time, and provides better regularity of the free-bounary (by one spatial derivative), and second, the space of equilibria is finite-dimensional and thus it is easier to understand the degrees-of-freedom that regulate the asymptotic state of the system, given the initial conditions.

1.6. Methodology and outline of the paper. Our present work builds on our new energy method for the Stefan problem that we developed in [29]. We obtain global and uniform 
control of the geometry of the free-boundary by controlling the weighted boundary-norm $\sup _{t \in[0, T]}\|\sqrt{\chi(t)} h\|_{6}$ for all $t \geq 0$. We are thus able to track the location of the moving free-boundary and measure its deviation from the initial state; this geometric control is strongly coupled to, and dependent upon, the exponential-in-time decay of the temperature function to zero.

There exist infinitely many steady states for the classical Stefan problem: for any sufficiently smooth hypersurface $\bar{\Gamma} \subset \mathbb{R}^{d}$, the pair $(\bar{p}, \bar{\Gamma}) \equiv(0, \bar{\Gamma})$ forms an equilibrium solution of the Stefan problem (1). This abundance of possible attractors for the long-time behavior of the solution $\Gamma(t)$ creates a conceptual difficulty in approaching the question of "asymptotic" convergence.

We address the temporal asymptotics by requiring our initial surface to be a small perturbation of the reference sphere. We use the energy spaces introduced in [29]; moreover, we do not expect to observe any decay for the height of the moving surface in this norm. Rather, given the expectation that the solution does converge to some nearby shape (so that $h$ remains small), we expect the temperature $q(t)$ to converge to zero exponentially fast, since it is a solution of the nonlinear heat equation 9a). Returning to the definition of the energy space $\mathscr{E}$ given in (11), we immediately encounter a potential problem for global-in-time estimates; specifically, the coefficient $-\partial_{N} q(t)$ in the energy expression $\int_{\Gamma}\left(-\partial_{N} q(t)\right)\left|\bar{\partial}^{6} h\right|^{2} d \theta$ is also expected to decay as $t \rightarrow \infty$ and it is $a$ priori unclear how to uniformly-in-time control the regularity of the boundary height function $h$. To understand the relationship between the decay of $q(t)$ and the smallness of $\mathscr{E}$, we will analyze the dynamics in three different and coupled regimes.

High-order energy estimates. We do not expect the height function $h(t)$ to decay to 0 as $t \rightarrow \infty$; rather, we expect $h(t)$ to remain close to the initial height function $h_{0}$. Assuming, without loss of generality, that $h_{0}=0$, to guarantee the smallness of $h-h_{0}=h$ we will prove that

$$
\begin{aligned}
\sup _{0 \leq s \leq t} \mathscr{E}(s)+\int_{0}^{t} \mathscr{D}(s) d s & \leq \mathscr{E}(0)+\sup _{0 \leq s \leq t} P\left(E_{\beta}\right) \mathscr{E}(s)+\delta \int_{0}^{t} \mathscr{D}(s) d s \\
& \leq \mathscr{E}(0)+O\left(\varepsilon_{0}\right) \sup _{0 \leq s \leq t} \mathscr{E}(s)+\delta \int_{0}^{t} \mathscr{D}(s) d s
\end{aligned}
$$

where $P$ is some polynomial function of the low-norm $E_{\beta}$. The above estimate yields an $a$ priori bound on $\mathscr{E}$ if $\varepsilon, \delta$ and $\mathscr{E}(0)$ are sufficiently small.

However, to close the higher-order energy estimates and thus obtain (24), we must contend with a very problematic integral (or error term) given by

$$
\mathscr{N}:=-\int_{0}^{T} \int_{\Gamma} \partial_{N} q_{t}\left|\bar{\partial}^{6} h\right|^{2} d \theta d t
$$

Driven by intuition from the linear heat equation, we expect $\partial_{N} q_{t}$ to decay exactly as fast as $-\partial_{N} q$. Comparing $\mathscr{N}$ to the energy contribution $\int_{\Gamma}\left(-\partial_{N} q\right)\left|\partial^{6} h\right|^{2}$ above, we note that $\mathscr{N}$ cannot be controlled by $\mathscr{E}$, as it is the same order as $\mathscr{E}$. Hence, to bound $\mathscr{N}$, we prove that after a sufficiently long time has elapsed, the quantity $\partial_{N} q_{t}$ turns strictly positive and hence $\mathscr{N}$ can be bounded from above by zero. In Lemma 4.2 we will quantify the meaning of "sufficiently long" time $t=T_{K}$ from the previous sentence, expressing it as a function of the ratio $K=\left\|q_{0}\right\|_{4} /\left\|q_{0}\right\|_{0}$.

More precisely, we break the total time interval into a (possibly long) transient interval $\left[0, T_{K}\right]$ and $\left[T_{K}, \infty\right)$. On the transient time-interval $\left[0, T_{K}\right]$ we $d o$ treat $\mathscr{N}$ as an error term, and by choosing $\mathscr{E}(0)$ sufficiently small, a straightforward application of a Gronwall-type 
inequality verifies that the interval of existence is greater than $T_{K}$, as explained in our proof of the main theorem (given Section 4.4). The bound for $\mathscr{N}$ grows exponentially with time, and as such, cannot be used to establish global-in-time estimates. Instead, a significantly more refined analysis is employed on the time-interval $\left[T_{K}, \infty\right)$, wherein we prove in Lemma 4.2 the negativity of $\mathscr{N}$ for $t=T_{K}$ and then use a maximum principle-type argument to guarantee the negativity for all $t \geq T_{K}$.

Exponential decay-in-time of the temperature function $q$. The last inequality in (24) holds only if $E_{\beta}$ itself remains small; in fact we will prove that as $t \rightarrow \infty,\|q(t)\|_{4}^{2}$ has the nearly optimal decay rate

$$
e^{-\left(2 \lambda_{1}-C \varepsilon_{0}\right) t},
$$

where $\lambda_{1}$ denotes the smallest eigenvalue of the Dirichlet-Laplacian on the unit disk. Moreover, the parabolic estimate we prove, will be roughly of the form

$$
\partial_{t} E_{\beta}+D \leq C\left(\varepsilon_{0}+\left\|q_{0}\right\|_{4} \frac{e^{-\beta t / 2}}{\chi(t)^{1 / 2}}\right) D,
$$

where the norms $E_{\beta}$ and $D$ have been defined in (15). A nice consequence of our analysis is that the potentially growing term, $\frac{e^{-\beta t / 2}}{\chi(t)^{1 / 2}}$, in fact remains small and decays in time. Next, we explain why this is true.

Lower bound for the velocity of the free boundary. We may think of the presence of the denominator $\frac{1}{\chi(t)^{1 / 2}}$ in the estimate (26) as a possible obstruction to controlling the regularity of $h$ and thus potentially preventing uniform ellipticity bounds for the parabolic operator 9a). To deal with this issue, we need a quantitative lower bound on the decay rate of $\chi(t)$. Moreover, this lower bound has to favorably compare to the size of $e^{-\beta t}$. With some extra work, such a Hopf-type inequality is implied by a result of Oddson [41], which leads to the lower-bound

$$
\chi(t) \gtrsim c_{1} e^{-\left(\lambda_{1}+c \varepsilon_{0}\right) t},
$$

where $c>0$ denotes a generic constant, and as before $c_{1}=\int_{\Omega} q_{0} \varphi_{1}$ is the first coefficient in the eigenfunction expansion of the initial datum $q_{0}$ with respect to the $L^{2}$-orthonormal eigenbasis of the Dirichlet-Laplacian on the unit disk. Finally, combining (25) and 27), we will show in Lemma 2.3 , that for small initial data,

$$
\left\|q_{0}\right\|_{4} \frac{e^{-\beta t / 2}}{\chi(t)^{1 / 2}} \lesssim \sqrt{\varepsilon_{0}} e^{-\gamma^{*} t}
$$

for some positive constant $\gamma^{*}$.

The result of Oddson [41] relies on a good choice of a barrier function that, combined with a maximum principle, allows for very precise information on the decay rate. That choice is, however, only one possible choice of a comparison function, and it is possible that there are different ones since [41] gives nearly sharp decay rate only in a nearly radial regime. If nearly radial, it is possible that in a viscosity or weak solution framework, one can use comparison principle arguments to deduce that "no-thin tentacles" form (cf. [31] which is in spirit close to [44], but again relies on presence of the forcing term) and the moving boundary remains in an annulus of width $O(\varepsilon)$. To that end, but in absence of forcing, the ideas from [4, 11, 44] may be very valuable - they would require a construction of an adaptive family of comparison functions that yield precise decay rates as time evolves. In forthcoming work, we plan to address the Stefan problem on arbitrary domains diffeomorphic to the unit ball, as well as the case of the two-phase Stefan problem. In both 
instances and not unrelated to the above discussion, we shall need a better, new choice of barrier functions related to the existence of so-called half-eigenvalues for the extremal Pucci operators in order to get the sharp decay rates. In particular, our approach is insensitive to the convexity properties of the initial domain, but it requires sufficient regularity.

Another advantage of the techniques developed in this paper is that it provides a general and robust framework for addressing the global stability questions for related free boundary problems in fluid mechanics in absence of surface tension.

Plan of the paper. In Section 2, we introduce the bootstrap assumptions and obtain various a priori estimates, that allow us to control low norms of the boundary function $h$ as well as the decaying low-norm $E_{\beta}$, and also establish the equivalence between the energies and the norms as mentioned earlier in the introduction (Section 2.5). In Section 3, we state energy identities and then perform the energy estimates. Finally in Section 4 we prove the main theorem. In Section 5, we discuss the modifications required for the analysis in three space dimensions, and for initial height functions $h_{0} \neq 0$. Appendix $\mathrm{A}$ is devoted to the proof of the energy identities stated in Section 3 The very short Appendix B provides a simple proof for the upper bound of $\partial_{N} q_{t}$.

\section{BOOTSTRAP ASSUMPTIONS AND a priori BOUNDS}

Let us assume that the solution $(q, h)$ to the Stefan problem (9) exists on some time interval $[0, T], T>0$, which is guaranteed by [29]. With the positive constant $\varepsilon_{0}<\varepsilon \ll 1$ to be specified later, we make the following bootstrap assumptions:

(smallness)

$$
\sup _{0 \leq s \leq T} \mathscr{E}(s)+\int_{0}^{t} \mathscr{D}(s) d s \leq \varepsilon^{2}, \quad \sup _{0 \leq s \leq T} E_{\beta}(s)+\int_{0}^{t} D(s) d s \leq \tilde{C} E_{\beta}(0)
$$

(lower-bound)

$$
\chi(t) \gtrsim c_{1} e^{-\left(\lambda_{1}+\eta / 2\right) t},
$$

where we the definitions of $\mathscr{E}, \mathscr{D}, E_{\beta}$, and $D$ are provided in (11), (12), and (15), respectively. With $\beta$ given in (16), $\beta=2 \lambda_{1}-\eta$, the bootstrap assumption (29b) can be written as $\chi(t) \gtrsim c_{1} e^{-(\beta / 2+\eta) t}$. Moreover, $\eta>0$ is a fixed small constant and it will be shown in the proof of the main theorem, Section 4.4 that $\eta$ must be chosen smaller than $1 / \sqrt{C \ln K}$ for some universal constant $C$. Note that since $E_{\beta}(0) \leq \varepsilon^{2}$, 29a implies the decay estimate $\|q\|_{4}^{2} \leq \varepsilon^{2} e^{-\beta t}$. Recall that the constant $c_{1}$ in the estimate (29b) is defined as $\int_{\Omega} q_{0}(x) \varphi_{1}(x) d x$.

We now briefly explain the logic of the proof of global existence that will be carried out in Section 4 If $\mathscr{T}$ is defined to be the maximal time at which the solution $(q, h)$ exists and satisfies the bootstrap assumptions, the first objective is to show that the bootstrap assumptions 29a and 29b yield an improved smallness and lower-bound estimates at time $\mathscr{T}$. If $\mathscr{T}$ were finite, by the local-in-time well-posedness theory and continuity of our norms we can extend the solution to an interval $\mathscr{T}+T^{*}$, while preserving the bootstrap assumptions (29a) and (29b), thus arriving at contradiction to the definition of $\mathscr{T}$. Hence $\mathscr{T}$ must be infinite.

It remains to show that for $\varepsilon$ chosen small enough, the smallness and the lower-bound estimates can indeed be improved. In Corollary 2.14 we will show that the assumption 29b is in fact improved, and in Lemma 4.1 we show that the assumption on $E_{\beta}+$ $\int_{0}^{T} D$ in 29a is also improved. Finally, in Section 4.4 we will prove that the smallness of $\mathscr{E}+\int_{0}^{t} \mathscr{D}$ assumed in (29a) is also preserved. Thus the smallness regime introduced 
through 29a - 29b will be shown to remain preserved by the dynamics of 9 for $\varepsilon>0$ chosen sufficiently small.

2.1. Poincaré-type inequality. Because the first eigenfunction $\varphi_{1}$ of the Dirichlet-Laplacian is positive in $\Omega$, while the remaining eigenfunctions oscillate about zero, it will be necessary to introduce a constant into our estimates which gives a measure of the initial temperature distribution in the first mode of the dynamics. To this end, we will make use of the following

Lemma 2.1. For $k \geq 3$, let $f \in H^{k}(\Omega) \cap H_{0}^{1}(\Omega), f: \Omega \rightarrow \mathbb{R}^{+}$be a strictly positive function on the interior of $\Omega$. Let $\varphi_{1}$ be the first eigenvector of the Dirichlet-Laplacian on the unit ball $B_{1}(0)=\Omega$. Then there exists a universal constant $C^{*}$ such that

$$
\|f\|_{0}^{2} \leq C^{*}\left(\int_{\Omega} f(x) \varphi_{1}(x) d x\right)\|f\|_{3} .
$$

Proof. We have that

$$
\int_{\Omega} f^{2} d x \leq \max _{x \in \Omega} \frac{f(x)}{\varphi_{1}(x)} \int_{\Omega} f \varphi_{1} d x .
$$

Since $-\frac{\partial \varphi_{1}}{\partial N}(x) \geq c>0$ for all $x \in \Gamma$, the higher-order Hardy inequality (Lemma 1 in [18]) together with the Sobolev embedding theorem shows that

$$
\max _{x \in \Omega} \frac{f(x)}{\varphi_{1}(x)} \leq C\left\|\frac{f}{\varphi_{1}}\right\|_{2} \leq C\|f\|_{3}
$$

which proves the lemma.

Corollary 2.2. Let $q_{0} \in H^{4}(\Omega) \cap H_{0}^{1}(\Omega)$ with $q_{0}>0$ in $\Omega$. We consider the eigenfunction expansion $q_{0}=\sum_{j=1}^{\infty} c_{j} \varphi_{j}$ of $q_{0}$ with respect to the $L^{2}$-orthonormal basis $\left\{\varphi_{1}, \varphi_{2}, \ldots\right\}$ consisting of the Dirichlet-Laplacian eigenfunctions on the unit disk $B_{1}(0)=\Omega$. Then, if $\frac{\left\|q_{0}\right\|_{4}}{\left\|q_{0}\right\|_{0}} \leq K$, it follows in particular that

$$
\frac{\left|c_{j}\right|}{c_{1}}<K, \quad j=1,2, \ldots
$$

Lemma 2.3. If the bootstrap assumptions (29a), 29b) hold, then

$$
\frac{E_{\beta}^{1 / 2}(t) e^{-\beta t / 2}}{\chi(t)^{1 / 2}} \leq \frac{\tilde{C}^{1 / 2} E_{\beta}(0)^{1 / 2} e^{-\beta t / 2}}{\chi(t)^{1 / 2}} \lesssim \sqrt{\varepsilon} e^{-\gamma t / 2}
$$

where $\gamma=\frac{\beta}{2}-\eta>0$.

Proof. By 29b), we have that

$$
\begin{aligned}
\frac{E_{\beta}(t)^{1 / 2} e^{-\beta t / 2}}{\chi(t)^{1 / 2}} & \leq C \frac{e^{-\beta t / 2}}{e^{-\left(\lambda_{1} / 2+\eta / 4\right) t}} \frac{E_{\beta}(0)^{1 / 2}}{c_{1}^{1 / 2}} \\
& \leq C e^{-\gamma t / 2} \frac{\left\|q_{0}\right\|_{4}}{c_{1}^{1 / 2}} \leq C K\left\|q_{0}\right\|_{4}^{1 / 2} e^{-\gamma t / 2} \leq C \sqrt{\varepsilon} e^{-\gamma t / 2},
\end{aligned}
$$

where we have used the fact that $c_{1}^{1 / 2} \gtrsim \frac{1}{K^{1 / 2}}\left\|q_{0}\right\|_{0}^{1 / 2}$ and $\left\|q_{0}\right\|_{4} \lesssim K\left\|q_{0}\right\|_{0}$. We have also used the bound $K\left\|q_{0}\right\|_{4}^{1 / 2} \leq C \sqrt{\varepsilon}$ (since $\varepsilon_{0}<\varepsilon$ ), as well as the smallness assumption (22) so that $K\left\|q_{0}\right\|_{4}^{1 / 2} \lesssim K \varepsilon_{0} / F(K)^{1 / 2} \leq C \varepsilon$. Note that $\gamma$ is explicitly given by $\gamma=\left(\frac{\beta}{2}-\eta\right)>0$, and that $\eta \ll \lambda_{1} / 2$. 


\subsection{A priori bounds on $h$.}

Lemma 2.4 (Suboptimal decay bound for $h_{t}$ ). Under the bootstrap assumptions (29a) and (29b), the following decay bound holds:

$$
\left|h_{t}\right|_{2.5} \lesssim \varepsilon e^{-\gamma t / 2}
$$

Proof. Differentiating equation (5), the Sobolev embedding theorem together with the fact that $h \geq 0$ (by the maximum principle) show that

$$
\begin{aligned}
\left|h_{t}\right|_{1} & \lesssim|v|_{W^{1, \infty}}+|h|_{2}|v|_{2}+|h|_{1}|v|_{1}|h|_{1} \\
& \lesssim|v|_{2}+|h|_{2}|v|_{2}+|h|_{1}|v|_{1}\left(\left|h_{0}\right|_{1}+t \sup _{0 \leq s \leq t}\left|h_{t}\right|_{1}\right),
\end{aligned}
$$

where we have used the fundamental theorem of calculus for the last inequality. Using the bootstrap assumption (29a), we see that $|v(t)| \lesssim e^{-\beta t}$, while thanks to Lemma 2.3 and the fact that $\sqrt{\mathscr{E}} \lesssim \varepsilon_{0}<\varepsilon$,

$$
|h|_{2}|v|_{2} \lesssim \sqrt{\chi}|h|_{2} \frac{|v|_{1}}{\sqrt{\chi}} \lesssim \frac{\sqrt{E_{\beta}}}{\sqrt{\chi}} \sqrt{\mathscr{E}} e^{-\beta t / 2} \lesssim \varepsilon e^{-\gamma t / 2} .
$$

Hence,

$$
\sup _{0 \leq s \leq t}\left|h_{t}\right|_{1} \lesssim \varepsilon e^{-\beta t / 2}+\varepsilon e^{-\gamma t / 2}\left(1+\sup _{0 \leq s \leq t}\left|h_{t}\right|_{1}\right),
$$

and with $\varepsilon>0$ sufficiently small, we see that

$$
\sup _{0 \leq s \leq t}\left|h_{t}\right|_{1} \lesssim \varepsilon e^{-\gamma t / 2} \lesssim \varepsilon
$$

Taking more derivatives of (5), the Sobolev embedding theorem shows that for $k=2,3$,

$$
\left|h_{t}\right|_{k} \leq|v|_{k}+\left|\frac{h_{\theta}}{1+h}\right|_{L^{\infty}}|v|_{k}+\left|\frac{h_{\theta}}{1+h}\right|_{k}|v|_{L^{\infty}} \lesssim|v|_{k}+\left|h_{\theta}\right|{ }_{1}|v|_{k}+\left|\frac{h_{\theta}}{1+h}\right|_{k}|v|_{1}
$$

where we have again used the fact that $h \geq 0$. Since

$$
\left|\frac{h_{\theta}}{1+h}\right|_{k} \lesssim|h|_{k+1}\left(1+P\left(|h|_{k-1}\right)\right), \quad k=2,3,
$$

for some polynomial function $P$, and since $|h|_{k} \leq\left|h_{0}\right|_{k}+t \sup _{0 \leq s \leq t}\left|h_{t}\right|_{k}$, we see that

$$
\left|\frac{h_{\theta}}{1+h}\right|_{k} \lesssim|h|_{k+1}\left(1+P(t) P\left(\sup _{0 \leq s \leq t}\left|h_{t}\right|_{k-1}\right)\right) .
$$

We now use (34) and 33) to infer that

$$
\left|h_{t}\right|_{k} \lesssim|v|_{k}\left(1+\sup _{0 \leq s \leq t}\left|h_{t}\right|_{2}\right)+|h|_{k+1}|v|_{1}\left(1+P(t) P\left(\sup _{0 \leq s \leq t}\left|h_{t}\right|_{2}\right)\right),
$$

where we have used $\left|h_{\theta}\right|_{1} \lesssim t \sup _{0 \leq s \leq t}\left|h_{t}\right|_{2}$. Interpolating between $k=2$ and $k=3$ yields

$$
\left|h_{t}\right|_{2.5} \lesssim|v|_{2.5}\left(1+\sup _{0 \leq s \leq t}\left|h_{t}\right|_{2}\right)+|h|_{2.5}|v|_{1}\left(1+P(t) P\left(\sup _{0 \leq s \leq t}\left|h_{t}\right|_{2}\right)\right) .
$$

and as above, Lemma 2.3 provides us with the inequality $|h|_{2.5}|v|_{1} \lesssim \varepsilon e^{-\gamma t / 2}$, which together with the bootstrap assumption (29a) shows that

$$
\sup _{0 \leq s \leq t}\left|h_{t}\right|_{2.5} \lesssim \varepsilon e^{-\beta t / 2}\left(1+\sup _{0 \leq s \leq t}\left|h_{t}\right|_{2.5}\right)+\varepsilon e^{-\gamma t / 2}\left(1+P(t) P\left(\sup _{0 \leq s \leq t}\left|h_{t}\right|_{2}\right)\right)
$$

and therefore with $\varepsilon>0$ sufficiently small,

$$
\sup _{0 \leq s \leq t}\left|h_{t}\right|_{2.5} \lesssim \varepsilon e^{-\beta t / 2}+\varepsilon e^{-\gamma t / 2}\left(1+P\left(\sup _{0 \leq s \leq t}\left|h_{t}\right|_{2}\right)\right)
$$


where the polynomial $P(t)$ has been absorbed in some universal constant due to the exponentially decaying factor $e^{-\gamma t / 2}$. On the other hand, the inequality (35) with $k=2$ together with the estimate (32) shows that $\left|h_{t}\right|_{2} \lesssim \varepsilon$ so that with (37), we conclude the proof.

Remark 2.5. Note that the estimate (31) can be stated more precisely, by keeping track of constant $c_{1}$ on the right-hand side, in which case,

$$
\left|h_{t}\right|_{2.5} \lesssim \varepsilon^{1 / 2} \sqrt{c_{1}} e^{-\gamma t / 2} .
$$

The proof follows from the last line of the proof of Lemma2.4 since $E_{\beta}(0)^{1 / 2} \leq K^{2} c_{1}$, due to the bound $\|q\|_{4} \leq K\left\|q_{0}\right\| \leq K^{2} c_{1}$. Note that $\sqrt{\varepsilon}$ on the right-hand side of (30) can be replaced by $\sqrt{c_{1}}$ for the same reason.

Lemma 2.6 (Smallness of the height function). Let $c_{1}=\int_{\Omega} q_{0} \varphi_{1} d x$ and suppose that the bootstrap assumptions (29a), (29b) hold. For $\varepsilon>0$ taken sufficiently small,

$$
\sup _{0 \leq s \leq t}|h(s)|_{4.5} \lesssim \sqrt{\varepsilon}
$$

while for lower-order norms,

$$
\sup _{0 \leq s \leq t}|h(s)|_{2.5} \lesssim c_{1} \text { and } \sup _{0 \leq s \leq t}|h(s)|_{4} \lesssim \varepsilon^{1 / 2} c_{1}^{1 / 4} .
$$

Proof. Observe that

$|h|_{2.5}^{2} \leq 2 \int_{0}^{t}|h|_{2.5}\left|h_{s}\right|_{2.5} d s \leq \sup _{0 \leq s \leq t}|h(s)|_{2.5} \int_{0}^{t}\left|h_{s}\right|_{2.5} d s \lesssim \sup _{0 \leq s \leq t}|h(s)|_{2.5} \int_{0}^{t} \varepsilon^{1 / 2} \sqrt{c_{1}} e^{-\gamma t / 2}$,

where we have used $(38)$ in the last bound. Taking the supremum over the time interval $[0, t]$ we deduce

$$
\sup _{0 \leq s \leq t}|h(s)|_{2.5} \lesssim \varepsilon^{1 / 2} \sqrt{c_{1}} .
$$

Using the well-known interpolation estimate (see, for example, [1])

$$
|f|_{k} \leq|f|_{l}^{\theta}|f|_{m}^{1-\theta}, \quad \theta=\frac{m-k}{m-l}, l \leq k \leq m,
$$

with $k=3, l=2.5, m=4$, and the fact that $\left|\sqrt{\chi} \bar{\partial}^{4} h_{t}\right|_{0}^{2}$ is bounded by $\mathscr{E}$, we have that

$$
\begin{aligned}
\left|h_{t}\right|_{3} & \lesssim\left|h_{t}\right|_{4}^{1 / 3}\left|h_{t}\right|_{2.5}^{2 / 3} \lesssim \frac{\mathscr{E}^{1 / 6}}{\chi(t)^{1 / 6}} \varepsilon^{1 / 3} c_{1}^{1 / 3} e^{-\gamma t / 3} \\
& \lesssim \varepsilon^{2 / 3} c_{1}^{1 / 6} e^{-\gamma^{*} t}
\end{aligned}
$$

where $\gamma^{*}=-\frac{1}{3} \gamma+\frac{1}{6}\left(\frac{\beta}{2}+\frac{\eta}{2}\right)=-\frac{1}{6} \beta+\frac{5 \eta}{12}>0$ (by definition, $\left.\gamma=-\frac{\beta}{2}-\eta\right)$. As a consequence,

$$
|h|_{3}^{2} \lesssim \int_{0}^{t}|h|_{3}\left|h_{t}\right|_{3} \lesssim \sup _{0 \leq s \leq t}|h(s)|_{3} \int_{0}^{t}\left|h_{t}(s)\right|_{3} d s \lesssim \varepsilon \sup _{0 \leq s \leq t}|h(s)|_{3} .
$$

Upon taking the supremum over the inetrval $[0, t]$, we finally have that

$$
\sup _{0 \leq s \leq t}|h(s)|_{3} \lesssim \varepsilon
$$

We can now improve the decay result of Lemma 2.4 first for the quantity $\left|h_{t}\right|_{2}$. Simply using the bound (42), exactly as in the proof of Lemma 2.4, we infer the improved estimate

$$
\left|h_{t}\right|_{2} \lesssim\|v\|_{2.5}\left(1+|h|_{3}\right) \lesssim c_{1} e^{-\beta t / 2} .
$$


As an immediate consequence, we obtain the smallness bound for $\sup _{0 \leq s \leq t}|h(s)|_{4}$ :

$$
\begin{aligned}
\int_{\Gamma}\left|\bar{\partial}^{4} h\right|^{2} d \theta & =\int_{0}^{t} \int_{\Gamma} \bar{\partial}^{4} h \bar{\partial}^{4} h_{t} d \theta d s=\int_{0}^{t} \int_{\Gamma} \bar{\partial}^{6} h \bar{\partial}^{2} h_{t} d \theta d s \\
& \leq \int_{0}^{t}\left|\bar{\partial}^{6} h\right|_{0}\left|\bar{\partial}^{2} h_{t}\right|_{0} d s \lesssim \int_{0}^{t}\left(\frac{\mathscr{E} 1 / 2}{\chi(s)^{1 / 2}} c_{1} e^{-\beta s / 2}\right) d s \\
& \lesssim \int_{0}^{t} \varepsilon \sqrt{c_{1}} e^{-\gamma_{s} / 2} d s \lesssim \varepsilon \sqrt{c_{1}} .
\end{aligned}
$$

Note that (44), in particular, implies the second bound in (40). Next, we establish the a priori smallness of $\sup _{0 \leq s \leq t}|h(s)|_{4.5}$. Thanks to (44), we improve the decay bound for $\left|h_{t}\right|_{2.5}$ in an analogous fashion to the improved decay estimate (43) for $\left|h_{t}\right|_{2}$. We obtain $\left|h_{t}\right|_{2.5} \lesssim c_{1} e^{-\beta t / 2}$. The first bound in (40) now follows from the fundamental theorem of calculus and the previous bound. A straightforward interpolation argument for fractional Sobolev spaces on the unit circle $\Gamma$, shows

$$
|h|_{4.5}^{2} \lesssim \int_{0}^{t}|h|_{6}\left|h_{t}\right|_{3} d s
$$

Using the interpolation estimate (41), with $l=2.5, k=3$, and $m=5$, we see that

$$
\left|h_{t}\right|_{3} \leq C\left|h_{t}\right|_{2.5}^{4 / 5}\left|h_{t}\right|_{5}^{1 / 5} \text {. }
$$

Using (46) with (45), and using the above bound on $\left|h_{t}\right|_{2.5}$, yields

$$
\begin{aligned}
|h|_{4.5}^{2} & \lesssim \int_{0}^{t}|h|_{6}\left|h_{t}\right|_{2.5}^{4 / 5}\left|h_{t}\right|_{5}^{1 / 5} d s \lesssim \int_{0}^{t} \frac{\mathscr{E} 1 / 2}{\chi(s)^{1 / 2}} c_{1}^{4 / 5} e^{-2 \beta s / 5}\left|h_{t}\right|_{5}^{1 / 5} d s \\
& \lesssim \varepsilon c_{1}^{3 / 10} \int_{0}^{t} e^{-\bar{\gamma}_{s}}\left|h_{t}\right|_{5}^{1 / 5} d s,
\end{aligned}
$$

where we also used the bootstrap assumption (29b). One checks that $\bar{\gamma}=-\frac{2 \beta}{5}+\left(\frac{\beta}{4}+\frac{\eta}{2}\right)=$ $\frac{3}{20} \beta-\frac{\eta}{2}>0$. We thus have

$$
|h|_{4.5}^{2} \lesssim \varepsilon c_{1}^{3 / 10} \int_{0}^{t} e^{-\bar{\gamma} s / 2} \times\left(e^{-\bar{\gamma} s / 2}\left|h_{t}\right|_{5}^{1 / 5}\right) d s .
$$

Hölder's inequality with $p=\frac{10}{9}$ and $q=10$ then shows that

$$
\begin{aligned}
|h|_{4.5}^{2} & \lesssim \varepsilon c_{1}^{3 / 10}\left(\int_{0}^{t}\left(e^{-\bar{\gamma} s / 2}\right)^{10 / 9} d s\right)^{9 / 10}\left(\int_{0}^{t}\left(e^{-5 \bar{\gamma} s}\left|h_{t}\right|_{5}^{2} d s\right)^{1 / 10}\right. \\
& \lesssim \varepsilon c_{1}^{3 / 10}\left(\int_{0}^{t} e^{-5 \bar{\gamma} s}\left|h_{t}\right|_{5}^{2} d s\right)^{1 / 10} \lesssim \varepsilon^{6 / 5} c_{1}^{1 / 5}
\end{aligned}
$$

where the last inequality follows from the definition of $\bar{\gamma}$ above, the bootstrap assumptions (29b) and 29a, and the estimate

$$
\begin{aligned}
\int_{0}^{t} e^{-5 \bar{\gamma}_{s}}\left|h_{t}\right|_{5}^{2} d s & \lesssim \int_{0}^{t} \frac{1}{c_{1}} e^{-5 \bar{\gamma}_{s}+(\beta / 4+\eta / 2) s} \inf _{\Gamma}\left(-\partial_{N} q(s)\right)\left|h_{t}\right|_{5}^{2} d s \\
& \lesssim \int_{0}^{t} \frac{1}{c_{1}} e^{-(\beta / 2+3 \eta) s} \inf _{\Gamma}\left(-\partial_{N} q(s)\right)\left|h_{t}\right|_{5}^{2} d s \\
& \lesssim \frac{1}{c_{1}} \int_{0}^{t} \int_{\Gamma}\left(-\partial_{N} q(s)\right)\left|\bar{\partial}^{5} h_{t}\right|^{2} d \theta d s \leq \frac{\varepsilon^{2}}{c_{1}}
\end{aligned}
$$


2.3. Differentiation rules for $A$. Since $A=[D \Psi]^{-1}$, it follows that

$$
\partial_{t} A_{i}^{k}=-A_{r}^{k} w^{r}{ }_{s} A_{i}^{s} ; \quad \bar{\partial} A_{i}^{k}=-A_{r}^{k} \bar{\partial} \Psi^{r}{ }_{s} A_{i}^{s} .
$$

In particular, a simple application of the above identities and the product rule imply that for any given $a, b \in \mathbb{N}$,

$$
\begin{aligned}
\bar{\partial}^{a} \partial_{t}^{b} A_{i}^{k} & =-A_{r}^{k} \bar{\partial}^{a} \partial_{t}^{b} \Psi^{r}{ }_{, s} A_{i}^{s}+\left\{\bar{\partial}^{a} \partial_{t}^{b}, A_{i}^{k}\right\}, \\
\left\{\bar{\partial}^{m} \partial_{t}^{n}, A_{i}^{k}\right\} & :=\sum_{l+l^{\prime} \geq 1} a_{l, l^{\prime}} \bar{\partial}^{l} \partial_{t}^{l^{\prime}}\left(A_{r}^{k} A_{i}^{s}\right) \bar{\partial}^{m-l} \partial_{t}^{n-l^{\prime}} \Psi^{r},
\end{aligned}
$$

where the term $\{\cdot, \cdot\}$ is the commutator error. Here the constants $a_{l, l^{\prime}}$ are some universal constants, depending only on $m, n, l$ and $l^{\prime}$ (where $0 \leq l \leq m, 0 \leq l^{\prime} \leq n$ ).

2.4. Estimates for $\nabla \Psi-\mathrm{Id}$ and $A-\mathrm{Id}$. Under assumption (29a), the elliptic estimate (7) shows that on the time-interval $[0, T]$,

$$
\|\nabla \Psi-\mathrm{Id}\|_{L^{\infty}\left(B_{1}\right)} \leq C\|\nabla \Psi-\mathrm{Id}\|_{1.5} \leq C|h|_{2}
$$

and for $0 \leq s \leq 3$,

Estimate (48) implies that

$$
\left\|D^{2} \Psi\right\|_{s} \leq C|h|_{s+1.5} .
$$

$$
\|A-\operatorname{Id}\|_{L^{\infty}\left(B_{1}\right)}=\|(\operatorname{Id}-\nabla \Psi) A\|_{L^{\infty}\left(B_{1}\right)} \leq C\|A\|_{L^{\infty}\left(B_{1}\right)}|h|_{2} ;
$$

thus under assumption (29a),

$$
\|A-\mathrm{Id}\|_{L^{\infty}\left(B_{1}\right)} \leq C|h|_{2}
$$

Note that (48) and (49) together imply that for $0 \leq s \leq 3$,

$$
\|D A\|_{s} \leq C|h|_{s+1.5} .
$$

Thus, with Lemma2.6, we have proven the following

Lemma 2.7. With the bootstrap assumptions (29a), 29b) and for $\varepsilon>0$ taken sufficiently small,

$$
\|\nabla \Psi-\mathrm{Id}\|_{4}+\|A-\mathrm{Id}\|_{4} \lesssim \sqrt{\varepsilon}
$$

2.5. High-order derivatives of $q$. Because our energy function $\mathscr{E}(t)$ is formed using only tangential derivatives in space, the purpose of this section is show that radial derivatives of the temperature $q$ are also bounded, and thus the full Sobolev norms of the temperature $q$ are controlled by our energy function, as was explained in the introduction.

We will make use of the heat equation and its time-differentiated variants:

$$
\begin{aligned}
q_{t}-\Delta_{\Psi} q & =f_{0}, \\
q_{t t}-\Delta_{\Psi} q_{t} & =f_{1} \\
q_{t t t}-\Delta_{\Psi} q_{t t} & =f_{2},
\end{aligned}
$$

where $\Delta_{\Psi}=A_{i}^{j} \frac{\partial}{\partial x_{j}}\left(A_{i}^{k} \frac{\partial}{\partial x_{k}}\right)$ and where the forcing functions $f_{0}, f_{1}, f_{2}$ are given by

$$
\begin{aligned}
& f_{0}=-\Psi_{t} \cdot v, \\
& f_{1}=-\left(\Psi_{t} \cdot v\right)_{t}+A_{i}^{j}\left(\partial_{t} A_{i}^{k} q_{, k}\right),{ }_{j}+\partial_{t} A_{i}^{j}\left(A_{i}^{k} q_{, k}\right),{ }_{j}, \\
& \begin{aligned}
f_{2}=-\left(\Psi_{t} \cdot v\right)_{t t}+2 A_{i}^{j}\left(\partial_{t} A_{i}^{k} q_{t, k}\right),{ }_{j}+2 \partial_{t} A_{i}^{j}\left(A_{i}^{k} q_{t, k}\right),{ }_{j}+2 \partial_{t} A_{i}^{j}\left(\partial_{t} A_{i}^{k} q_{, k}\right),{ }_{j} \\
\quad \quad+\partial_{t}^{2} A_{i}^{j}\left(A_{i}^{k} q_{t, k}\right),{ }_{j}+A_{i}^{j}\left(\partial_{t}^{2} A_{i}^{k} q_{, k}\right), j .
\end{aligned}
\end{aligned}
$$

We will repeatedly make use of the following elliptic estimate: 
Lemma 2.8 (Elliptic regularity with Sobolev-class coefficients). Let $q$ denote the unique $H_{0}^{1}(\Omega)$ solution to

$$
\begin{aligned}
-\Delta \Psi q=F & \text { in } \Omega, \\
q=0 & \text { on } \partial \Omega .
\end{aligned}
$$

Suppose that $k>1, F \in H^{k-1}(\Omega)$, and $A \in H^{k}(\Omega)$ satisfying $A_{i}^{k} A_{i}^{j} \xi_{j} \xi_{k} \geq \lambda|\xi|^{2}$ for all $\xi \in \mathbb{R}^{2}$ for some $\lambda>0$. Then

$$
\|q\|_{H^{k+1}(\Omega)} \leq C\left[\|F\|_{H^{k-1}(\Omega)}+\|A\|_{H^{k}(\Omega)}^{p}\|F\|_{L^{2}(\Omega)}\right]
$$

for some power $p>1$.

Proof. We provide the details in the course of the proof of Lemma 2.9

Lemma 2.9 (Bounding $\partial_{t}^{l} q, l=0,1,2,3$, by $\left.\mathscr{E}(t)\right)$. With the bootstrap assumptions (29a) and (29b) holding, and with $\varepsilon>0$ sufficiently small, there exists a constant $C^{*}$ such that

$$
\left\|q_{t t t}\right\|_{0}^{2}+\left\|q_{t t}\right\|_{2}^{2}+\left\|q_{t}\right\|_{4}^{2}+\|q\|_{6}^{2} \leq C^{*} \mathscr{E} .
$$

Proof. Step1. Estimating $\left|h_{t t t}\right|_{0.5}$. We denote by $\mathscr{X}(t)$ the quantity $\left\|q_{t t t}\right\|_{0}^{2}+\left\|q_{t t}\right\|_{2}^{2}+$ $\left\|q_{t}\right\|_{4}^{2}+\|q\|_{6}^{2}$. Twice time-differentiating $\underline{9 \mathrm{~d}}$, we find that

$$
h_{t t t}=v_{t t} \cdot N-\left[\frac{h_{\theta}}{1+h}\right]_{t t} v \cdot \tau-2\left[\frac{h_{\theta}}{1+h}\right]_{t} v_{t} \cdot \tau-\frac{h_{\theta}}{1+h} v_{t t} \cdot \tau .
$$

By the normal trace theorem (see, for example, equation (6.1) in [18]),

$$
\left|v_{t t} \cdot N\right|_{0.5} \lesssim\left\|\bar{\partial} v_{t t}\right\|_{0}^{2}+\left\|\operatorname{div} v_{t t}\right\|_{0}^{2}
$$

Note that

(52)

$\operatorname{div} v_{t t}=(\operatorname{div} \Psi v)_{t t}+((\operatorname{div}-\operatorname{div} \Psi) v)_{t t}=\left(q_{t}+v \cdot \Psi_{t}\right)_{t t}+\left[\left(A_{i}^{k}-\delta_{i}^{k}\right) v^{i},_{k}\right]_{t t}=q_{t t t}+\Psi_{t t t} \cdot v+\mathscr{R}$,

where the remainder $\mathscr{R}$ reads

$$
\mathscr{R}=2 \Psi_{t t} \cdot v_{t}+\Psi_{t} \cdot v_{t t}+\left(A_{i}^{k}-\delta_{i}^{k}\right)_{t t} v^{i},{ }_{k}+2\left(A_{i}^{k}-\delta_{i}^{k}\right)_{t} v_{t, k}^{i}+\left(A_{i}^{k}-\delta_{i}^{k}\right) v_{t t, k}^{i} .
$$

From Lemma 2.6 and 2.3 , we obtain the estimate $\|\mathscr{R}\|_{0}^{2} \lesssim \varepsilon \mathscr{E}+\varepsilon \mathscr{X}$. Thus, returning to (52) and using that $\left\|q_{t t t}+\Psi_{t t t} \cdot v\right\|_{0}^{2} \leq \mathscr{E}$ by (11), we get $\left\|\operatorname{div} v_{t t}\right\|_{0}^{2} \lesssim \mathscr{E}+\varepsilon \mathscr{X}$ and consequently

$$
\left|v_{t t} \cdot N\right|_{0.5} \lesssim \mathscr{E}+\varepsilon \mathscr{X} .
$$

As for the last term on the right-hand side of 51), we use the tangential trace theorem (see, for example, equation (6.2) in [18]) to infer that

$$
\left|v_{t t} \cdot \tau\right| \lesssim\left\|\bar{\partial} v_{t t}\right\|_{0}^{2}+\left\|\operatorname{curl} v_{t t}\right\|_{0}^{2}
$$

Since curl $\Psi v=0($ recall $v=-\nabla p \circ \Psi)$, we have $\operatorname{curl} v_{t t}=[(\operatorname{curl}-\operatorname{curl} \Psi) v]_{t t}$. By a similar inequality as above, using Lemmas 2.6 and 2.3 , we obtain the bound $\left\|\left[\left(\operatorname{curl}-\operatorname{curl}{ }_{\Psi}\right) v\right]_{t t}\right\|_{0}^{2} \lesssim$ $\varepsilon \mathscr{E}+\varepsilon \mathscr{X}$. Together with (53) and $\left\|\partial v_{t t}\right\|_{0}^{2} \leq \mathscr{E}$, this leads to

$$
\left|v_{t t} \cdot \tau\right|_{0.5} \lesssim \mathscr{E}+\varepsilon \mathscr{X} .
$$

Together with the smallness of $h_{\theta}$ and $h_{\theta t}$ from Lemma 2.6 , the bound $\left|\sqrt{\chi} \bar{\partial} h_{t t}\right|_{1}^{2} \leq \mathscr{E}$ and Lemma 2.3. we finally infer from (51) that

$$
\left|h_{t t t}\right|_{0.5} \lesssim \mathscr{E}+\varepsilon \mathscr{X} .
$$


Step 2: $L^{2}$ estimates for $\partial_{t}^{l} q$. By the triangle inequality and the definition 111 of $\mathscr{E}(t)$, we have that for $l=1,2,3$,

$$
\begin{aligned}
\left\|\partial_{t}^{l} q\right\|_{0}^{2} & \leq\left\|\partial_{t}^{l} q+\partial_{t}^{l} \Psi \cdot v\right\|_{0}^{2}+\left\|\partial_{t}^{l} \Psi \cdot v\right\|_{0}^{2} \\
& \leq \mathscr{E}(t)+\left\|\partial_{t}^{l} \Psi \cdot v\right\|_{0}^{2} \\
& \lesssim \mathscr{E}(t)+\|v\|_{3}^{2}\left\|\partial_{t}^{l} \Psi\right\|_{0}^{2} \lesssim \mathscr{E}(t)+\varepsilon^{2}\left|h_{t t t}\right|_{0.5}^{2} \\
& \lesssim \mathscr{E}(t)+\varepsilon \mathscr{X},
\end{aligned}
$$

where we used the Sobolev embedding theorem and 54].

Step 3: $H^{2}$ estimate for $q_{t t}$. We consider the elliptic equation $-\Delta \Psi q=f_{0}-q_{t}$. We note that Lemma2.7 ensures that $A_{i}^{k} A_{i}^{j} \xi_{k} \xi_{j} \geq \frac{1}{2}|\xi|^{2}$ for all $\xi \in \mathbb{R}^{2}$. Given that $\left\|f_{0}-q_{t}\right\|_{0}^{2} \lesssim \mathscr{E}$, elliptic estimates show that $\|q\|_{2}^{2} \lesssim \mathscr{E}$. This, in turn, implies that $\left\|f_{1}-q_{t t}\right\|_{0}^{2} \lesssim \mathscr{E}$, and elliptic estimates then show that $\left\|q_{t}\right\|_{2}^{2} \lesssim \mathscr{E}$. Hence, we have that $\left\|f_{2}-q_{t t t}\right\|_{0}^{2} \lesssim \mathscr{E}+\varepsilon \mathscr{X}$, and once again use elliptic estimates to conclude that $\left\|q_{t t}\right\|_{2}^{2} \lesssim \mathscr{E}+\varepsilon \mathscr{X}$.

Step 4: $H^{4}$ estimate for $q_{t}$. Since $\left\|f_{0}-q_{t}\right\|_{2}^{2} \lesssim \mathscr{E}$, Lemma 2.8 shows that $\|q\|_{4}^{2} \lesssim \mathscr{E}$; thus, $\left\|f_{1}-q_{t t}\right\|_{2}^{2} \lesssim \mathscr{E}+\varepsilon \mathscr{X}$. Another application of Lemma 2.8 together with Lemma2.7t then shows that $\left\|q_{t}\right\|_{4}^{2} \lesssim \mathscr{E}+\varepsilon \mathscr{X}$.

Step 5: $H^{6}$ estimate for $q$. The elliptic estimates in Steps 3 and 4 made use of Lemma2.7 To obtain the $H^{6}$ estimate for $q$ requires us to improve the elliptic estimate in Lemma 2.8 to be linear in $\|\sqrt{\chi} \Psi\|_{6}$. To this end, we write $\mathscr{A}^{j k}=A_{i}^{j} A_{i}^{k}$ and rewrite (50) as

$$
-\left(\mathscr{A}^{j k} q_{, k}\right),{ }_{j}=-q_{t}+f_{0}-A_{i}^{j},{ }_{j} A_{i}^{k} q_{, k} .
$$

Letting $\bar{\partial}^{\alpha}$ act on (55), we find that $\bar{\partial}^{\alpha} q$ satisfies

$$
\begin{gathered}
-\left[\mathscr{A}^{i j}\left(\bar{\partial}^{\alpha} q\right),{ }_{j}\right],{ }_{i}=-\bar{\partial}^{\alpha}\left(\Psi_{t} \cdot v+q_{t}\right)+\sum_{0<\beta \leq \alpha} C_{\alpha \beta}\left[\left(\bar{\partial}^{\beta} \mathscr{A}^{i j}\right)\left(\bar{\partial}^{\alpha-\beta} q\right),{ }_{j}\right],{ }_{i} \\
-\sum_{0 \leq \beta<\alpha} C_{\alpha \beta} \bar{\partial}^{\beta}\left(A_{i}^{j},{ }_{j} A_{i}^{k}\right) \bar{\partial}^{\alpha-\beta} q,{ }_{j},
\end{gathered}
$$

where $C_{\alpha \beta}$ are constants from the product rule. Multiplying this equation with $\bar{\partial}^{\alpha} q$ and integrating-by-parts, using the fact that $\bar{\partial}^{\alpha} q=0$ on $\partial \Omega$ and that $\mathscr{A} \geq 1 / 2$, we find that

$$
\begin{aligned}
& \frac{1}{2}\left\|\bar{\partial}^{\alpha} q\right\|_{1}^{2} \leq\left\|\bar{\partial}^{\alpha-1}\left(\Psi_{t} \cdot v+q_{t}\right)\right\|_{0}\left\|\bar{\partial}^{\alpha+1} q\right\|_{0}+\sum_{0<\beta \leq \alpha} C_{\alpha \beta}\left\|\left(\bar{\partial}^{\beta} \mathscr{A}^{i j}\right)\left(\bar{\partial}^{\alpha-\beta} q\right),{ }_{j}\right\|_{0}\left\|\bar{\partial}^{\alpha} q, i\right\|_{0} \\
& +\sum_{0 \leq \beta<\alpha} C_{\alpha \beta}\left\|\bar{\partial}^{\beta}\left(A_{i}^{j},{ }_{j} A_{i}^{k}\right) \bar{\partial}^{\alpha-\beta} q,\right\|_{0}\left\|\bar{\partial}^{\alpha}{ }^{\alpha}\right\|_{0}+\left\|\bar{\partial}^{\alpha-1}\left(A_{i}^{j},{ }_{j} A_{i}^{k}\right) \bar{\partial} q,{ }_{j}\right\|_{0}\left\|\bar{\partial}^{\alpha} q\right\|_{1} .
\end{aligned}
$$

Let us examine the second term on the right-hand side of (56). By Young's inequality, for $\delta>0$

$\sum_{0<\beta \leq \alpha} C_{\alpha \beta}\left\|\left(\bar{\partial}^{\beta} \mathscr{A}^{i j}\right)\left(\bar{\partial}^{\alpha-\beta} q\right),{ }_{j}\right\|_{0}\left\|\bar{\partial}^{\alpha} q_{, i}\right\|_{0} \leq \delta\left\|\bar{\partial}^{\alpha} q\right\|_{1}^{2}+C_{\delta} \sum_{0<\beta \leq \alpha} C_{\alpha \beta}\left\|\bar{\partial}^{\beta} \mathscr{A} \bar{\partial}^{\alpha-\beta} D q\right\|_{0}^{2}$

where $C_{\delta}=C / \delta$. Since $\bar{\partial}^{5} \mathscr{A} \sim \bar{\partial}^{5} D \Psi P(A)+\bar{\partial}^{4} D \Psi P(\bar{\partial} D \Psi, A)+\bar{\partial}^{3} D \Psi P\left(\bar{\partial}^{2} D \Psi, \bar{\partial} D \Psi, A\right)$, it thus follows that for $\alpha=4$ or 5 ,

$$
\left\|\bar{\partial}^{\alpha} \mathscr{A}\right\|_{0} \leq C\left\|\bar{\partial}^{\alpha-2}(\Psi-e)\right\|_{3}^{2} \leq C \frac{|\sqrt{\chi} h|_{5.5}^{2}}{\chi} \lesssim \frac{\mathscr{E}}{\chi}
$$


The linear inequality (57) shows that our bootstrap assumptions 29a and 29b imply that the map $h \mapsto \mathscr{A}$ is linear with respect to these high norms.

We first consider the case that $\alpha=4$. From (57) when $\alpha=\beta=4$

$$
\left\|\bar{\partial}^{\alpha} \mathscr{A} D q\right\|_{0}^{2} \lesssim \frac{E_{\beta} e^{-\beta t}}{\chi} \mathscr{E} \lesssim \varepsilon e^{-\gamma t} \mathscr{E} .
$$

The Cauchy-Schwarz inequality, together with the Sobolev embedding theorem, shows that $\left\|\bar{\partial}^{3} \mathscr{A} \bar{\partial} D q\right\|_{0}^{2}$ has the same bound. Next, $\left\|\bar{\partial}^{2} \mathscr{A} \bar{\partial}^{2} D q\right\|_{0}^{2}+\left\|\bar{\partial} \mathscr{A} \bar{\partial}^{3} D q\right\|_{0}^{2} \lesssim\|\Psi\|_{4}^{2}\|q\|_{4}^{2} \lesssim$ $\varepsilon e^{-\beta t} \mathscr{E} \lesssim \varepsilon e^{-\gamma t} \mathscr{E}$.

The first, third, and fourth terms on the right-hand side of (56) are estimated in a similar fashion, so we do not provide the details. Hence, by choosing $\delta>0$ sufficiently small and employing Young's inequality, we find that

$$
\|q\|_{4}^{2}+\sum_{\alpha \leq 4}\left\|\bar{\partial}^{\alpha} q\right\|_{1}^{2} \lesssim \mathscr{E}+\varepsilon \mathscr{X}
$$

To estimate radial derivatives, we use polar coordinates for the disc (with the usual basis $\mathbf{e}_{\mathbf{r}}$ and $\mathbf{e}_{\theta}$ ). Expressing the components of the matrix $\mathscr{A}$ as

$$
\mathscr{A}=\left[\begin{array}{ll}
\mathscr{A}^{r r} & \mathscr{A}^{r \theta} \\
\mathscr{A}^{\theta r} & \mathscr{A}^{\theta \theta}
\end{array}\right],
$$

we may write

$$
\operatorname{div}(\mathscr{A} \nabla q)=r^{-1}\left(r \mathscr{A}^{r r} q_{r}\right)_{r}+r^{-1}\left(\mathscr{A}^{r \theta} q_{\theta}\right)_{r}+r^{-1}\left(\mathscr{A}^{r \theta} q_{r}\right)_{\theta}+r^{-1}\left(r^{-1} \mathscr{A}^{\theta \theta} q_{\theta}\right)_{\theta} .
$$

It follows that

$$
\begin{gathered}
-\mathscr{A}^{r r} \bar{\partial}^{\alpha} q_{r r}=r^{-1}\left(r \mathscr{A}^{r r}\right)_{r} \bar{\partial}^{\alpha} q_{r}+r^{-1}\left(\mathscr{A}^{r \theta} \bar{\partial}^{\alpha} q_{r}\right)_{r}+r^{-1}\left(\mathscr{A}^{r \theta} \bar{\partial}^{\alpha} q_{r}\right)_{\theta}+r^{-1}\left(r^{-1} \mathscr{A}^{\theta \theta} \bar{\partial}^{\alpha} q_{\theta}\right)_{\theta} \\
-\bar{\partial}^{\alpha}\left(\Psi_{t} \cdot v+q_{t}\right)+\sum_{0<\beta \leq \alpha} C_{\alpha \beta}\left[\left(\bar{\partial}^{\beta} \mathscr{A}^{i j}\right)\left(\bar{\partial}^{\alpha-\beta} q\right),{ }_{j}\right], i \\
-\sum_{0 \leq \beta \leq \alpha} C_{\alpha \beta} \bar{\partial}^{\beta}\left(A_{i}^{j}, A_{i}^{k}\right) \bar{\partial}^{\alpha-\beta} q_{, j},
\end{gathered}
$$

Let $\omega=\left\{x \in \Omega: \frac{1}{2}<|x|<1\right\}$. For $\alpha \leq 3$, every term on the right-hand side has $L^{2}(\omega)$-norm bounded by a constant multiple of $\mathscr{E}$. Hence, it follows that

$$
\sum_{\alpha \leq 3}\left\|\bar{\partial}^{\alpha} q\right\|_{2, \omega}^{2} \lesssim \mathscr{E}+\varepsilon \mathscr{X}
$$

Allowing $\frac{\partial}{\partial r}$ to act on 59 , as many as three times, we conclude that

$$
\|q\|_{5, \omega}^{2} \lesssim \mathscr{E}+\varepsilon \mathscr{X} .
$$

We return to the inequality 56 and consider the case that $\alpha=5$. Once again, we focus on the second term on the right-hand side, the first and third terms being similar (and easier). From (58) $\left\|\bar{\partial}^{5} \mathscr{A} D q\right\|_{0}^{2} \lesssim \varepsilon e^{-\gamma t} \mathscr{E}$. The Cauchy-Schwarz inequality, together with the Sobolev embedding theorem, shows that $\left\|\bar{\partial}^{4} \mathscr{A} \bar{\partial} D q\right\|_{0}^{2}+\left\|\bar{\partial}^{3} \mathscr{A}^{2} \bar{\partial}^{2} D q\right\|_{0}^{2}+$ $\left\|\bar{\partial}^{2} \mathscr{A} \bar{\partial}^{3} D q\right\|_{0}^{2} \lesssim \varepsilon e^{-\gamma t} \mathscr{E}$. Finally, using (60), we conclude $\left\|\bar{\partial} \mathscr{A} \bar{\partial}^{4} D q\right\|_{0}^{2} \lesssim \varepsilon\|v\|_{4}^{2} \lesssim$ $\varepsilon e^{-\gamma t} \mathscr{E}$. We conclude that

$$
\|q\|_{4}^{2}+\sum_{\alpha \leq 5}\left\|\bar{\partial}^{\alpha} q\right\|_{1}^{2} \lesssim \mathscr{E}+\varepsilon \mathscr{X}
$$

Then setting $\alpha=0$ and letting $\frac{\partial^{4}}{\partial r^{4}}$ act on (59) shows that indeed

$$
\|q\|_{6, \omega}^{2} \lesssim \mathscr{E}+\varepsilon \mathscr{X} .
$$


By using a smooth cut-off function whose support contains $\overline{\Omega \backslash \omega}$, we easily obtain the interior estimates, and find that $\|q\|_{6}^{2} \lesssim \mathscr{E}=\varepsilon \mathscr{X}$. Recalling the definition of $\mathscr{X}$ and the estimates from Steps 2, 3, and 4, we finally infer $\mathscr{X} \lesssim \mathscr{E}$, which concludes the proof of the lemma.

Lemma 2.10 (Bounding $\partial_{t}^{l} q, l=0,1,2,3$ by $\mathscr{D}(t)$ ). With the bootstrap assumptions (29a) and (29b), and for $\varepsilon>0$ sufficiently small, there exists a $\gamma>0$ such that

$$
\sum_{l=0}^{2}\left\|\partial_{t}^{l} q_{t}\right\|_{5-2 l}^{2}+\|q\|_{6.5}^{2} \lesssim \varepsilon e^{-\gamma t} \mathscr{E}+\mathscr{D} .
$$

Corollary 2.11. With the bootstrap assumptions (29a), (29b) and a sufficiently small $\varepsilon>0$,

$$
\|v\|_{5.5}^{2}+\left|h_{t}\right|_{5}^{2} \lesssim \varepsilon e^{-\gamma t} \mathscr{E}+\mathscr{D}
$$

with $\gamma=\beta / 2-\eta$ as defined in Lemma 2.3

Proof of Corollary 2.11. We write $9 \mathrm{~b}$ as

$$
v=D q \cdot(\operatorname{Id}-A)-D q
$$

Using the basic estimate from Lemma 2.7, we see that

$$
\begin{gathered}
\|v\|_{5} \lesssim(1+\sqrt{\varepsilon})\|q\|_{6}+E_{\beta}^{\frac{1}{2}} e^{-\beta t / 2}\|\Psi-e\|_{6}, \\
\|v\|_{6} \lesssim(1+\sqrt{\varepsilon})\|q\|_{7}+E_{\beta}^{\frac{1}{2}} e^{-\beta t / 2}\|\Psi-e\|_{7},
\end{gathered}
$$

so that an application of linear interpolation (see, for example, Theorem 7.17 in Adams [1]) provides the inequality

$$
\|v\|_{5.5}^{2} \lesssim(1+\sqrt{\varepsilon})\|q\|_{6.5}^{2}+E_{\beta}\|\Psi-e\|_{6.5}^{2} .
$$

Using Lemmas 2.3 and 2.10, it follows that

$$
\begin{aligned}
\|v\|_{5.5}^{2} & \lesssim(1+\sqrt{\varepsilon})\|q\|_{6.5}^{2}+\frac{E_{\beta}(t) e^{-\beta t / 2}}{\chi(t)} \chi(t)\|\Psi-e\|_{6.5}^{2} \\
& \lesssim(1+\sqrt{\varepsilon})\|q\|_{6.5}^{2}+\frac{E_{\beta}(t) e^{-\beta t / 2}}{\chi(t)} \mathscr{E}(t) \\
& \lesssim \varepsilon^{2} e^{-\gamma t} \mathscr{E}+\mathscr{D} .
\end{aligned}
$$

Next, using the formula (5), we see that

$$
\left|h_{t}\right|_{5}^{2} \lesssim \chi(t)|h|_{6}^{2} \frac{|v|_{2.5}^{2}}{\chi(t)}+\varepsilon|v|_{5}^{2}
$$

which once again, thanks to Lemmas 2.3 and 2.10 is bounded by a constant multiple of $\varepsilon^{2} e^{-\gamma t} \mathscr{E}+\mathscr{D}$.

Proof of Lemma 2.10 Step 1: $H^{1}$ estimates for $\partial_{t}^{l} q$. We make use of the identity $\nabla q=$ $v \cdot \nabla \Psi$. It follows that

$$
\begin{aligned}
\nabla q_{t} & =v_{t} \cdot \nabla \Psi+v \cdot \nabla \Psi_{t} \\
\nabla q_{t t} & =v_{t t} \cdot \nabla \Psi+2 v_{t} \cdot \nabla \Psi_{t}+v \cdot \nabla \Psi_{t t} \\
\nabla q_{t t t} & =v_{t t t} \cdot \nabla \Psi+3 v_{t t} \cdot \nabla \Psi_{t}+3 v_{t} \cdot \nabla \Psi_{t t}+v \cdot \nabla \Psi_{t t t}
\end{aligned}
$$


Employing Hölder's inequality and the Sobolev embedding theorem,

$$
\left\|\nabla q_{t t t}\right\|_{0}^{2} \lesssim\left\|v_{t t t}\right\|_{0}^{2}|h|_{2}^{2}+\left\|v_{t t}\right\|_{0}^{2}\left|h_{t}\right|_{2}^{2}+\frac{\left\|v_{t}\right\|_{2}^{2}}{\chi}\left|\sqrt{\chi} h_{t t}\right|_{0}^{2}+\frac{\|v\|_{2}^{2}}{\chi}\left|\sqrt{\chi} h_{t t t}\right|_{0}^{2} \lesssim \mathscr{D}
$$

where we have used Lemma 30 for the last inequality. We have similar estimates for $q_{t t}$, $q_{t}$, and $q$ so that

$$
\sum_{l=0}^{3}\left\|\partial_{t}^{l} q\right\|_{1}^{2} \lesssim \mathscr{D}
$$

Step 2. $H^{3}$ estimate for $q_{t t}$. Just as in the proof of Corollary 2.11, we see that as a consequence of Lemma2.9.

$$
\sum_{l=0}^{2}\left\|\partial_{t}^{l} v\right\|_{5-2 l} \lesssim \mathscr{E}
$$

Returning to the equation (50), we estimate $-\Psi_{t} \cdot v-q_{t}$ in $H^{1}(\Omega)$. By the Sobolev embedding theorem together with Lemmas 2.4 and 2.3

$$
\left\|\Psi_{t}\right\|_{W^{1, \infty}} \lesssim\left\|\Psi_{t}\right\|_{3} \lesssim \sqrt{\varepsilon} e^{-\gamma t / 2},
$$

so that together with [63, $\left\|\Psi_{t} \cdot v\right\|_{1}^{2} \lesssim \varepsilon e^{\gamma t} \mathscr{E}$. Then, with with [62),

$$
\|q\|_{3}^{2} \lesssim \varepsilon e^{-\gamma t} \mathscr{E}+\mathscr{D} .
$$

Next, we return to (50) and estimate $f_{1}-q_{t t}$ in $H^{1}(\Omega)$. By Lemma 2.4 $\left\|\Psi_{t} \cdot v_{t}\right\|_{1}^{2} \lesssim$ $\varepsilon e^{-\gamma t} \mathscr{E}$, while $\left\|\Psi_{t t} \cdot v\right\|_{1}^{2} \lesssim \frac{\mathscr{E} e^{-\beta t}}{\chi} E_{\beta} \lesssim \varepsilon e^{-\gamma t} \mathscr{E}$. The estimates 62) and (65) then show that $\left\|f_{1}-q_{t t}\right\|_{1}^{2} \lesssim \varepsilon e^{-\gamma t} \mathscr{E}+\mathscr{D}$ so that

$$
\left\|q_{t}\right\|_{3}^{2} \lesssim \varepsilon e^{-\gamma t} \mathscr{E}+\mathscr{D}
$$

A similar estimate then shows that $\left\|f_{2}-q_{t t t}\right\|_{1}^{2} \lesssim \varepsilon e^{-\gamma t} \mathscr{E}+\mathscr{D}$ so that from (50),

$$
\left\|q_{t t}\right\|_{3}^{2} \lesssim \varepsilon e^{-\gamma t} \mathscr{E}+\mathscr{D} \text {. }
$$

Step 3. $H^{5}$ estimate for $q_{t}$. From (63) and (64), we see that $\left\|\Psi_{t} \cdot v\right\|_{3}^{2} \lesssim \varepsilon e^{-\gamma t} \mathscr{E}+\mathscr{D}$, so that with Lemmas 2.7 and 2.8, we have that

$$
\|q\|_{5}^{2} \lesssim \varepsilon e^{-\gamma t} \mathscr{E}+\mathscr{D} .
$$

This, in turn, ensures that $\left\|f_{1}-q_{t t}\right\|_{3}^{2} \lesssim \varepsilon e^{-\gamma t} \mathscr{E}+\mathscr{D}$ so that

$$
\left\|q_{t}\right\|_{5}^{2} \lesssim \varepsilon e^{-\gamma t} \mathscr{E}+\mathscr{D}
$$

Step 4. $H^{6.5}$ estimate for $q$. We first look at the estimate (56) with $\alpha=5$. We find that

$$
\begin{aligned}
\left\|\bar{\partial}^{5} q\right\|_{1} \lesssim & \left\|\Psi_{t} \cdot v\right\|_{4}+\left\|q_{t}\right\|_{4}+\sum_{0<\beta \leq 5}\left\|\left(\bar{\partial}^{\beta} \mathscr{A}^{i j}\right)\left(\bar{\partial}^{5-\beta} q\right), j\right\|_{0} \\
& +\sum_{0 \leq \beta<5}\left\|\bar{\partial}^{\beta}\left(A_{i}^{j},{ }_{j} A_{i}^{k}\right) \bar{\partial}^{\alpha-\beta} q, j\right\|_{0}+\left\|\bar{\partial}^{4}\left(A_{i}^{j},{ }_{j} A_{i}^{k}\right) \bar{\partial} q, j\right\|_{0}
\end{aligned}
$$


For the first term on the right-hand side, we note that with the Sobolev embedding theorem and Lemma 2.3 .

$$
\begin{aligned}
\left\|\Psi_{t} \cdot v\right\|_{k} & \lesssim\left\|\sqrt{\chi} \Psi_{t}\right\|_{k} \frac{\|v\|_{3}}{\sqrt{\chi}}+\left\|\Psi_{t}\right\|_{3}\|v\|_{k} \\
& \lesssim \sqrt{\varepsilon} e^{-\gamma t / 2}\left(\left\|\sqrt{\chi} \Psi_{t}\right\|_{k}+\|v\|_{k}\right) \quad k=4,5 .
\end{aligned}
$$

Using the estimate [57), we see that

$$
\sum_{0<\beta \leq 5}\left\|\left(\bar{\partial}^{\beta} \mathscr{A}^{i j}\right)\left(\bar{\partial}^{5-\beta} q\right),\right\|_{0} \lesssim \sqrt{\varepsilon} e^{-\gamma t / 2}\left(\|\sqrt{\chi}(\Psi-e)\|_{6}+\|q\|_{5}\right)
$$

The last two term on the right-hand side of (66) are estimated in the same way so that

$$
\left\|\bar{\partial}^{5} q\right\|_{1} \lesssim \sqrt{\varepsilon} e^{-\gamma t / 2}\left(\left\|\sqrt{\chi} \Psi_{t}\right\|_{4}+\|\sqrt{\chi}(\Psi-e)\|_{6}+\|v\|_{4}+\left\|q_{t}\right\|_{4}\right) .
$$

Using the formula $(59)$, we find that

$$
\|q\|_{6} \lesssim \sqrt{\varepsilon} e^{-\gamma t / 2}\left(\left\|\sqrt{\chi} \Psi_{t}\right\|_{4}+\|\sqrt{\chi}(\Psi-e)\|_{6}+\|v\|_{4}+\left\|q_{t}\right\|_{4}\right)+\left\|q_{t}\right\|_{4} .
$$

The identical procedure with $\alpha=6$ then yields

$$
\|q\|_{7} \lesssim \sqrt{\varepsilon} e^{-\gamma t / 2}\left(\left\|\sqrt{\chi} \Psi_{t}\right\|_{5}+\|\sqrt{\chi}(\Psi-e)\|_{7}+\|v\|_{5}+\left\|q_{t}\right\|_{5}\right)+\left\|q_{t}\right\|_{5} .
$$

Linear interpolation between (67) and [68), we have that

$$
\begin{aligned}
\|q\|_{6.5} & \lesssim \sqrt{\varepsilon} e^{-\gamma t / 2}\left(\left\|\sqrt{\chi} \Psi_{t}\right\|_{4.5}+\|\sqrt{\chi}(\Psi-e)\|_{6.5}+\|v\|_{4.5}+\left\|q_{t}\right\|_{4.5}\right)+\left\|q_{t}\right\|_{4.5} \\
& \lesssim \varepsilon e^{-\gamma t / 2} \mathscr{E}^{1 / 2}+\mathscr{D}^{1 / 2} .
\end{aligned}
$$

2.6. Lower bound on $\chi(t)$. The heat equation $9 \mathrm{a}$ for $q$ can be rewritten as

$$
\begin{aligned}
q_{t}-a_{k j} q_{, k j}-b_{k} q_{, k} & =0 \text { in } \Omega, \\
q & =0 \text { on } \Gamma, \\
q(0, \cdot) & =q_{0}>0 \text { in } \Omega
\end{aligned}
$$

where the coefficient matrix $a=\left(a_{k j}\right)_{k, j=1,2}$, and the vector $b=\left(b_{1}, b_{2}\right)$ are explicitly given by:

$$
a_{k j}:=A_{i}^{k} A_{i}^{j} ; \quad b_{k}:=A_{i, j}^{k} A_{i}^{j}+A_{i}^{k} \Psi_{t}^{i} .
$$

We first quote a theorem from [41], that will play an important role in producing quantitative bounds from below for $\chi(t)$.

Lemma 2.12 (Oddson's Theorem 2 in [41]). Let $q \in C^{1,2}(\Omega)$ be a supersolution to (69) in the unit disc $\Omega=B_{1}(\mathbf{0})$, and let $0<\alpha \leq \frac{1}{2}$ be the normalized ellipticity constant satisfying

$$
a_{j k} \xi_{j} \xi_{k} \geq \alpha\left(a_{11}+a_{22}\right)|\xi|^{2}
$$

for any real vector $\xi=\left(\xi_{1}, \xi_{2}\right)$. Moreover, let us introduce the quantities

$$
k_{0}(T):=\inf _{\Omega \times[0, T]} \frac{1}{a_{11}+a_{22}}, \quad \beta(T):=\sup _{\Omega \times[0, T]} b \cdot x .
$$

Let $J_{\mu}$ denote the Bessel function of the first kind of order $\mu$ and $\xi_{0}$ its first positive zero. If we define

$$
\mu=\frac{\beta+1}{2 \alpha}-1, \quad \lambda=\frac{\alpha \xi_{0}^{2}}{k_{0}},
$$


then there exists a positive constant $m$ satisfying

$$
q(t, x) \geq m \rho e^{-\lambda t}
$$

in $B_{1}(0) \times[\sigma, \infty[$, where $\rho$ stands for the distance from $x$ to the boundary $\Gamma$ and $\sigma$ is an arbitrary small time.

Remark 2.13 (Optimal decay rate for solutions of the heat equation). If we set $A=I d$, then problem (69) turns into the initial-boundary value problem for the linear heat equation. In this case $k_{0}=\frac{1}{2}, \alpha=1 / 2, \beta \equiv 0, \mu=\frac{0+1}{1}-1=0$, and $\lambda=\xi_{0}^{2}$, where $\xi_{0}$ stands for the first positive zero of $J_{0}(\xi)$. In particular, if $q^{\text {heat }}$ denotes the associated solution, then the above lemma implies that

$$
\chi_{\text {heat }}(t):=\inf _{x \in \Gamma}\left(-\partial_{N} q^{\text {heat }}(t, x)\right) \gtrsim e^{-\xi_{0}^{2} t}
$$

which is the optimal decay rate in the case of the linear heat equation, as the lowest positive eigenvalue of the Dirichlet-Laplacian on the two-dimensional disk corresponds exactly to

$$
\lambda_{1}=\xi_{0}^{2} .
$$

Corollary 2.14 (Lower-bound for $\chi(t))$. Under the bootstrap assumptions (29a) and (29b) with $\varepsilon$ small enough, there exists a universal constant $C>0$ such that

$$
\chi(t) \gtrsim c_{1} e^{-\left(\lambda_{1}+\tilde{\lambda}(t)\right) t},
$$

where $c_{1}=\int_{\Omega} q_{0} \varphi_{1} d x$ is the first coefficient in the eigenfunction expansion of the initial datum $q_{0}$ with respect to the $L^{2}$ ortho-normal basis $\left\{\varphi_{1}, \varphi_{2}, \ldots\right\}$ of the eigenvectors of the Dirichlet-Laplacian on $B_{1}(0)$, i.e $q_{0}=c_{1} \varphi_{1}+c_{2} \varphi_{2}+\ldots$ Moreover, $\tilde{\lambda}(t) \geq 0$ satisfying $\tilde{\lambda}(t) \leq C \varepsilon$ for some positive constant $C$. In particular, with $\varepsilon>0$ sufficiently small so that $C \varepsilon<\eta / 4$, we obtain the improvement of the bootstrap bound (29b) given by $\chi(t) \gtrsim$ $c_{1} e^{-\left(\lambda_{1}+\eta / 4\right) t}$.

Proof. The proof of Oddson's Theorem 2 in [41] (Lemma 2.12) relies on the construction of a comparison function of the form $v(t, r)=r^{-\mu} J_{\mu}\left(\xi_{0} r\right) e^{-\lambda t}$, where $\lambda, \mu, \xi_{0}$ are given in the statement of Lemma 2.12 $J_{\mu}$ is a Bessel function of the first kind and $r=|x|$ is the radial coordinate. The first property of $v$ which is important for the proof is that $v$ vanishes at the spatial boundary $\Gamma$ and approaches it like $c(1-r) e^{-\lambda t}$ as $r \rightarrow 1$. This is a consequence of the fact that $\lim _{r \rightarrow 1} \frac{J_{\mu}\left(\xi_{0} r\right)}{r^{\mu}(1-r)}=c$ for some constant $c>0$, a well known property of Bessel functions. The second important property is that $v$ is a subsolution for 69) (and it is constructed with the help of maximal Pucci operators as explained in detail in [41]).

The goal is to prove that for any arbitrarily small time $\sigma>0$ there exists a strictly positive constant $\delta(\sigma)>0$ such that $q-\delta v$ is a positive supersolution to the parabolic problem 69] on the time interval $[\sigma, \infty[$. The desired lower bound for $q$ then follows from the weak maximum principle.

Since $v$ is a subsolution, it follows that for any $\delta>0, q-\delta v$ is a supersolution. The positivity of $q-\delta v$ at $t=\sigma$ follows from the parabolic Hopf lemma, from which we infer the existence of a constant $\delta(\sigma)$ such that $\frac{q}{v}>\delta(\sigma)$ uniformly over $\bar{\Omega}$. Note that we have used the fact that $v(\sigma, r)$ behaves like $C(1-r)$ near the boundary $\Gamma$ for some positive constant $C$. Therefore it follows that the constant $m$ in the statement of Lemma 2.12 $a$ priori depends on the time $\sigma>0$, and moreover, $m$ is proportional to the lower bound for $-\partial q /\left.\partial N\right|_{t=\sigma}$ on $\Gamma$. 
From the proof of the parabolic Hopf lemma (see for instance Theorem 3.14 in [21]), the value $-\partial q /\left.\partial N\right|_{t=\sigma}$ is proportional to the minimal value of the temperature $q$ on a space-time region of the form $K_{\sigma}:=B_{1-C \sigma} \times[\sigma / 2,3 \sigma / 2]$, divided by $\sigma$ (which is roughly the distance of $K_{\sigma}$ from the parabolic boundary of $\Omega \times[0,2 \sigma]$ ). Note that, unlike the elliptic case, we are forced to take into account the time-dependence of the solution and in particular the region $K_{\sigma}$ cannot be chosen uniformly for all times, but only for times greater or equal some arbitrarily small $\sigma>0$. However, our solution is continuous all the way to $t=0$ and we do nevertheless obtain a lower bound for all times due to the Taylor sign condition; namely, due to (18),

$$
-\partial_{N} q_{0}=\frac{-\partial_{N} q_{0}}{c_{1}} c_{1} \gtrsim c_{1} .
$$

Note however that if we define the dimensionless quantity $L=\left(-\partial_{N} q_{0}\right) / c_{1}>0$ and assume no universal bound on $L$ from below, the only modification in the statement of the main theorem will be that the smallness condition on initial data 22 will additionally depend on $L$.

As to the bound on $\tilde{\lambda}$, note that the exponent $\lambda=\lambda\left(\left(a_{i j}\right),\left(b_{i}\right)\right)$ depends on the coefficients $\left(a_{i j}\right)_{i, j=1,2}$ and $\left(b_{i}\right)_{i=1,2}$ through the relationship $\lambda=\alpha \xi_{0}^{2} / k_{0}$. Since $k_{0}$ and $\xi_{0}$ vary continuously as the coefficients are varied, it proves that $\lambda$ depends continuously on the coefficients $a_{i j}, b_{i}$ of the parabolic operator. On the other hand, by Remark2.13 it follows $\left.\lambda\right|_{a_{i j}=\delta_{i j}, b_{i}=0}=\lambda_{1}$. As a consequence

$$
|\tilde{\lambda}(t)|=\left|\lambda(t)-\lambda_{1}\right| \leq C\left(\|A-\operatorname{Id}\|_{L^{\infty}},\|b\|_{L^{\infty}}\right)=O\left(\left\|D^{2}(\Psi-e)\right\|_{L^{\infty}},\left\|\Psi_{t}\right\|_{L^{\infty}}\right) .
$$

\section{ENERGY IDENTITY AND THE HIGHER-ORDER ENERGY ESTIMATE}

3.1. The energy identity. Much of our analysis is founded on basic higher-order energy identities for the classical Stefan problem. These identities provide the geometrical control of the evolving phase boundary, which in turn controls the decay of the temperature function; moreover, these identities explain our definition of the higher-order energy function $\mathscr{E}$ and the dissipation function $\mathscr{D}$.

Proposition 3.1 (Energy identity). With $R=1+h$ and $R_{J}=R J^{-1}$, sufficiently smooth solutions to the classical Stefan problem satisfy

$$
\begin{gathered}
\frac{d}{d t} \mathscr{E}(t)+\mathscr{D}(t)=\sum_{j=0}^{3}\left(\int_{\Gamma}\left(-\partial_{N} q_{t}\right) R_{J}^{2}\left|\bar{\partial}^{6-2 j} \partial_{t}^{j} h\right|^{2}+\int_{\Omega}\left(\mathscr{R}_{j}+\tilde{\mathscr{R}}_{j}\right)+\int_{\Gamma} \mathscr{G}_{j}\right) \\
+\sum_{j=1}^{3}\left(\int_{\Omega}\left(\mathscr{S}_{j}+\tilde{\mathscr{S}}_{j}\right)+\int_{\Gamma} \mathscr{H}_{j}\right),
\end{gathered}
$$

where the error terms $\mathscr{R}_{j}, \tilde{\mathscr{R}}_{j}, \mathscr{S}_{j}, \tilde{\mathscr{S}}_{j}, \mathscr{G}_{j}$, and $\mathscr{H}_{j}$ are given by A.14, A.15, A.23, A.27, A.16, and $A .24$, respectively.

The proof is provided in Appendix A

Remark 3.2. On the right-hand side of (71), we have isolated the error term

$$
\mathscr{G}_{\text {Hopf }}=\int_{\Gamma}\left(-\partial_{N} q_{t}\right) R_{J}^{2}\left|\bar{\partial}^{6-2 j} \partial_{t}^{j} h\right|^{2} d x^{\prime}
$$

from the other boundary-integral error terms $\mathscr{G}_{j}$ and $\mathscr{H}_{j}$; indeed, $\mathscr{G}_{\text {Hopf }}$ can only be thought of as an "error term" on a transient time-interval, for after a sufficiently large time, we will 
no longer be able to control $\mathscr{G}_{\text {Hopf }}$ via energy methods, and instead, we have to rely upon a Hopf-type argument to prove that $\mathscr{G}_{\mathrm{Hopf}}<0$.

3.2. Energy estimates. To control some of the highest-order error terms in our energy estimates, we shall make use of the following technical lemma, whose proof is given in [15] and [16].

Lemma 3.3. Let $H^{\frac{1}{2}}(\Omega)^{\prime}$ denote the dual space of $H^{\frac{1}{2}}(\Omega)$. There exists a positive constant C such that

$$
\|\bar{\partial} F\|_{\frac{1}{2}(\Omega)^{\prime}} \leq C\|F\|_{H^{\frac{1}{2}(\Omega)}} \text { for } F \in H^{\frac{1}{2}}(\Omega) .
$$

As a consequence of the energy identity (71), we can establish our fundamental energy inequality.

Proposition 3.4 (The energy estimate). Suppose that the bootstrap assumptions (29a) and (29b) hold with $\varepsilon>0$ and $\eta>0$ sufficiently small. Letting $K=\frac{\left\|q_{0}\right\|_{4}}{\left\|q_{0}\right\|_{0}}$,

$\sup _{0 \leq s \leq t} \mathscr{E}(s)+\frac{1}{2} \int_{0}^{t} \mathscr{D}(s) d s \leq \mathscr{E}(0)+C K^{2} \int_{0}^{t} e^{\eta s} \mathscr{E}(s) d s+O(\sqrt{\varepsilon}) \sup _{0 \leq s \leq t} \mathscr{E}(s)$ for $t \in[0, T]$.

Proof. Throughout the proof, we will rely on the a priori bounds of Section 2 in particular, we will often make use of Lemmas 2.3, 2.6, 2.9, and 2.10,

Step 1. The estimate for $\mathscr{G}_{\mathrm{Hopf}}$ in (72) We claim that

$$
\left|\mathscr{G}_{\mathrm{Hopf}}\right| \leq C K^{2} \int_{0}^{t} e^{\eta s} \mathscr{E}(s) d s .
$$

Note that

$$
\left.\left.\left|\int_{0}^{t} \int_{\Gamma}\left(-\partial_{N} q_{t}\right) R_{J}^{2}\right| \bar{\partial}^{6} h\right|^{2}|\leq C| \int_{0}^{t} \int_{\Gamma} \frac{\left(-\partial_{N} q_{t}\right)}{-\partial_{N} q}\left(-\partial_{N} q\right)\left|\bar{\partial}^{6} h\right|^{2}\left|\leq C \int_{0}^{t}\right| \frac{\partial_{N} q_{t}}{-\partial_{N} q}\right|_{L^{\infty}} \mathscr{E}(s) d s .
$$

In order to bound the term $\left|\frac{\partial_{N} q_{t}}{\partial_{N} q}\right|$, we need a decay estimate for the numerator $\left|\partial_{N} q_{t}\right|$. The Sobolev embedding theory would yield the bound $\left|\partial_{N} q_{t}\right|_{L^{\infty}} \lesssim\left\|q_{t}\right\|_{2+\delta}$ for $\delta>0$, but by definition of our decay norm $E_{\beta}$, it is only the $H^{2}(\Omega)$-norm of $q_{t}$ for which we have the desired decay. Thus, we arrive at the decay estimate for $q_{t}$ by using a comparison principle together with Theorem 1 in Oddson [41]; indeed, in Appendix B] we prove that

$$
\left|\partial_{N} q_{t}\right|_{L^{\infty}} \lesssim K^{2} c_{1} e^{-\beta t / 2}
$$

It then follows from the bootstrap assumption $29 \mathrm{~b}$ that

$$
\left|\frac{\partial_{N} q_{t}(s)}{-\partial_{N} q(s)}\right|_{L^{\infty}} \leq \frac{C K^{2} c_{1} e^{-\left(\lambda_{1}-\eta / 2\right) s}}{c_{1} e^{-\left(\lambda_{1}+\eta / 2\right) s}} \leq C K^{2} e^{\eta s},
$$

which, in turn, establishes (74).

Step 2. Estimates for $\mathscr{R}_{j}, \tilde{\mathscr{R}}_{j}$, and $\mathscr{G}_{j}$ in (71). Our objective will be to show that

$$
\left|\int_{0}^{t} \int_{\Omega}\left(\mathscr{R}_{j}+\tilde{\mathscr{R}}_{j}\right)+\int_{0}^{t} \int_{\Gamma} \mathscr{G}_{j}\right| \leq O(\sqrt{\varepsilon}) \sup _{0 \leq s \leq t} \mathscr{E}(s)+\delta \int_{0}^{t} \mathscr{D}(s) \text {, for } j=0, \ldots 3 .
$$

We establish (76) for the most difficult case, $j=0$. The case when $j=1,2$, or 3 can then be proven in a similar fashion. The proof for $j=0$ is divided into three parts, and we shall begin with the term $\mathscr{R}_{0}$. 
Estimates for the integral $\int_{\Omega} \mathscr{R}_{0}$. As derived in $\underline{\text { A.9 }}$, the term $\mathscr{R}_{0}$ can be written as

$$
\begin{gathered}
\mathscr{R}_{0}:=\underbrace{\mu \sum_{l=1}^{5} c_{l} \bar{\partial}^{l} A_{i}^{k} \bar{\partial}^{6-l} q_{, k} \bar{\partial}^{6} v^{i}}_{=: I_{1}}+\underbrace{\left(\mu q_{, k} A_{i}^{s} A_{r}^{k}\right), \bar{\partial}^{6} \Psi_{\kappa}^{r} \bar{\partial}^{6} v^{i}}_{=: I_{2}}+\underbrace{\mu\left\{\bar{\partial}^{6}, A_{i}^{k}\right\} q_{, k} \bar{\partial}^{6} v^{i}}_{=: I_{3}}+\underbrace{\mu A_{i}^{s} \bar{\partial}^{6} \Psi^{r} A_{r}^{k} q_{, k}\left\{\bar{\partial}^{6}, \partial_{s}\right\} v^{i}}_{=: I_{4}} \\
-\underbrace{\left(\mu A_{i}^{k}\right)_{, k} \bar{\partial}^{6} q^{6} v^{i}}_{=: I_{5}}-\underbrace{\mu A_{i}^{k}\left\{\bar{\partial}^{6}, \partial_{k}\right\} q \bar{\partial}^{6} v^{i}}_{=: I_{6}}-\mu \sum_{l=1}^{6}(\underbrace{c_{l} \bar{\partial}^{l} A_{i}^{k} \bar{\partial}^{6-l} v_{, k}^{i}\left(\bar{\partial}^{6} q+\bar{\partial}^{6} \Psi \cdot v\right)}_{=: I_{7}}
\end{gathered}
$$

A.9

$$
+\underbrace{d_{l} \bar{\partial}^{6-l} w \cdot \bar{\partial}^{l} v\left(\bar{\partial}^{6} q+\bar{\partial}^{6} \Psi \cdot v\right)}_{=: I_{8}}-\underbrace{\bar{\partial}^{6} \Psi \cdot v_{t}\left(\bar{\partial}^{6} q+\bar{\partial}^{6} \Psi \cdot v\right)}_{=: I_{9}}) .
$$

Estimate of $\int_{\Omega} I_{1}$. For the extremal case $l=5$,

$$
\begin{aligned}
\left|\int_{\Omega} \bar{\partial}^{5} A_{i}^{k} \bar{\partial} q_{, k} \bar{\partial}^{6} v^{i}\right| & \leq\left\|\bar{\partial}^{5} A_{i}^{k}\right\|_{L^{4}}\left\|\bar{\partial} q_{, k}\right\|_{L^{4}}\left\|\bar{\partial}^{6} v^{i}\right\|_{0} \\
& \lesssim\|\Psi-\operatorname{Id}\|_{6.5}\left\|\bar{\partial} q_{, k}\right\|_{0.5}\left\|\bar{\partial}^{6} v^{i}\right\|_{0} \\
& \lesssim|h|_{6}\|q\|_{4}\left\|\bar{\partial}^{6} v^{i}\right\|_{0} \\
& \lesssim \frac{\|q\|_{4}}{\chi(t)^{1 / 2}} \mathscr{E}^{1 / 2} \mathscr{D} \mathscr{D}^{1 / 2} \\
& \leq \frac{C}{\delta} e^{-\gamma t} \varepsilon \mathscr{E}+\delta \mathscr{D},
\end{aligned}
$$

where we have used Hölder's inequality and the Sobolev embedding theorem, as well as Young's inequality together with Lemma 2.3 for the last inequality.

If $l=4$, then Lemmas 2.6 and 2.10 and Corollary 2.11 show that

$$
\begin{aligned}
\left|\int_{\Omega} \bar{\partial}^{4} A_{i}^{k} \bar{\partial}^{2} q_{, k} \bar{\partial}^{6} v^{i}\right| & \leq\left\|\bar{\partial}^{4} A_{i}^{k}\right\|_{0}\left\|\bar{\partial}^{2} q_{, k}\right\|_{L^{\infty}}\left\|\bar{\partial}^{6} v^{i}\right\|_{0} \lesssim|h|_{4.5}\|q\|_{4.5} \mathscr{D}^{1 / 2} \\
& \lesssim \varepsilon\left(\|q\|_{4.5}^{2}+\mathscr{D}\right) \lesssim \varepsilon\left(\varepsilon e^{-\gamma t} \mathscr{E}+\mathscr{D}\right) \lesssim \varepsilon^{2} e^{-\gamma t} \mathscr{E}+\varepsilon \mathscr{D} .
\end{aligned}
$$

The case when $l=1,2$ or 3 are estimated in the same way and yield the same bound.

Estimates of $\int_{\Omega} I_{k}$ for $k=2,3,4,5$. The following estimate holds:

$$
\left|\int_{\Omega} I_{2}+I_{3}+I_{4}+I_{5}\right| \lesssim \frac{C}{\delta} \varepsilon e^{-\gamma t} \mathscr{E}+\delta \mathscr{D} .
$$

For the integral of $I_{2}$, an application of an $L^{\infty}-L^{2}-L^{2}$ Hölder's inequality together with Lemmas 2.3 and 2.6 leads to

$$
\begin{aligned}
\left|\int_{\Omega} I_{2}\right| & \lesssim\left\|\left(\mu q_{, k} A_{i}^{s} A_{r}^{k}\right)_{s}\right\|_{L^{\infty}}\left\|\bar{\partial}^{6} \Psi^{r}\right\|_{0}\left\|\bar{\partial}^{6} v^{i}\right\|_{0} \\
& \lesssim\left\|\mu A_{i}^{s} A_{r}^{k}\right\|_{W^{1, \infty}}\|q\|_{3} \frac{\mathscr{E}^{1 / 2}}{\chi^{1 / 2}} \mathscr{D}^{1 / 2} \lesssim \frac{C}{\delta} e^{-\gamma t} \varepsilon \mathscr{E}+\delta \mathscr{D} .
\end{aligned}
$$

The estimates for terms $I_{3}, I_{4}, I_{5}$, and $I_{6}$ are established in the same manner. Note that the commutator $\left\{\bar{\partial}^{6}, A_{i}^{k}\right\} q_{, k}$ in $I_{3}$ is defined in (47) ) and has at most five derivatives acting on $q_{, k}$; moreover, the expression $\left\{\bar{\partial}^{6}, \partial_{k}\right\} f=\bar{\partial}^{6} \partial_{k} f-\partial_{k} \bar{\partial}^{6} f$ is of the form $\sum_{1 \leq|\alpha| \leq 6} a_{\alpha} \partial_{\alpha} f$, where the $a_{\alpha}$ are smooth uniformly bounded functions on the set $\omega=\left\{x \in \Omega\left|\frac{1}{2} \leq\right| x \mid \leq 1\right\}$. 
Estimate $\int_{\Omega} I_{7}$. We first consider the case that $l=6$, and write

$$
\int_{\Omega} \bar{\partial}^{6} A_{i}^{k} v_{k}^{i}\left(\bar{\partial}^{6} q+\bar{\partial}^{6} \Psi \cdot v\right)=\underbrace{\int_{\Omega} \bar{\partial}^{6} A_{i}^{k} v_{k}^{i} \bar{\partial}^{6} q}_{J_{1}}+\underbrace{\int_{\Omega} \bar{\partial}^{6} A_{i}^{k} v_{k}^{i} \bar{\partial}^{6} \Psi \cdot v}_{J_{2}} .
$$

Thanks to Lemma 3.3, we see that $J_{1} \leq\left\|\bar{\partial}^{5} A\right\|_{0.5}\left\|D v \bar{\partial}^{6} q\right\|_{0.5}$. By linear interpolation and the Sobolev embedding theorem, $\left\|D v \bar{\partial}^{6} q\right\|_{0.5} \lesssim\|v\|_{3}\|q\|_{6}+\|v\|_{2.5}\|q\|_{6.5} \lesssim\|v\|_{3}\|q\|_{6.5}$. It thus follows that

$J_{1} \lesssim|h|_{6}\left\|_{5.5}\right\| v\left\|_{3}\right\| q\left\|_{6.5} \lesssim \frac{C}{\delta}|h|_{6}^{2}\right\| v\left\|_{3}^{2}+\delta\right\| q \|_{6.5}^{2} \lesssim \frac{C \mathscr{E} E_{\beta} e^{-\beta t}}{\delta \chi(t)} \lesssim \delta \mathscr{D}+\varepsilon e^{-\gamma t} \mathscr{E}+\delta\left(\varepsilon e^{-\gamma t} \mathscr{E}+\mathscr{D}\right)$,

for some positive constant $\gamma>0$, where we have employed Lemmas 2.3 and 2.10 with Corollary 2.11

As for the integral of $J_{2}$, we again use Lemma 3.3 to deduce that

$$
\left|\int_{\Omega} \bar{\partial}^{6} A_{i}^{k} v_{k}^{i} \bar{\partial}^{6} \Psi \cdot v\right| \leq\left\|\bar{\partial}^{5} A_{i}^{k}\right\|_{0.5}\left\|v_{, k}^{i} \bar{\partial}^{6} \Psi \cdot v\right\|_{0.5} \lesssim\|v\|_{2.5}^{2}\|\Psi-\operatorname{Id}\|_{6.5}^{2} \lesssim e^{-\beta t} E_{\beta} \frac{\mathscr{E}}{\chi(t)} \lesssim \varepsilon e^{-\gamma t} \mathscr{E},
$$

where $\gamma>0$ is given by Lemma 2.3 Now for the case that $l=5$ in the integral of the term $I_{7}$, it follows that

$$
\begin{aligned}
& \left|\int_{\Omega} \bar{\partial}^{5} A_{i}^{k} \bar{\partial} v_{, k}^{i}\left(\bar{\partial}^{6} q+\bar{\partial}^{6} \Psi \cdot v\right)\right| \leq\left\|\bar{\partial}^{5} A_{i}^{k}\right\|_{L^{4}}\left\|\bar{\partial} v_{, k}^{i}\right\|_{L^{4}}\left\|\bar{\partial}^{6} q+\bar{\partial}^{6} \Psi \cdot v\right\|_{0} \\
& \lesssim\left\|\bar{\partial}^{5} A_{i}^{k}\right\|_{0.5}\left\|\bar{\partial} v_{, k}^{i}\right\|_{0.5}\left\|\bar{\partial}^{6} q+\bar{\partial}^{6} \Psi \cdot v\right\|_{0} \lesssim \frac{\mathscr{E}^{1 / 2}}{\chi(t)^{1 / 2}}\|v\|_{2.5} \mathscr{E}^{1 / 2} \lesssim \frac{E_{\beta}^{1 / 2} e^{-\beta t / 2}}{\chi(t)^{1 / 2}} \mathscr{E} \lesssim \sqrt{\varepsilon} e^{-\gamma t} \mathscr{E},
\end{aligned}
$$

where we used Lemma 2.3 again and the fact that (by definition of $\mathscr{E}$ ), $\left\|\bar{\partial}^{6} q+\bar{\partial}^{6} \Psi \cdot v\right\|_{0}^{2} \lesssim$ $\mathscr{E}$. Hereby we used the estimate (30). The remaining cases $l=1,2,3,4$ follow analogously and the estimates rely on a systematic use of Lemmas 2.3, 2.6, 2.10, and Corollary 2.11

Estimate of $\int_{\Omega} I_{8}$. For the case that $l=1$ or 2 , we have that

$$
\begin{aligned}
\left|\int_{\Omega} \bar{\partial}^{6-l} w \cdot \bar{\partial}^{l} v\left(\bar{\partial}^{6} q+\bar{\partial}^{6} \Psi\right)\right| & \lesssim\left\|\bar{\partial}^{6-l} w\right\|_{0}\left\|\bar{\partial}^{l} v\right\|_{L^{\infty}}\left\|\bar{\partial}^{6} q+\bar{\partial}^{6} \Psi \cdot v\right\|_{0} \lesssim \frac{\mathscr{D}^{1 / 2}}{\chi(t)^{1 / 2}} E_{\beta}^{1 / 2} e^{-\beta t / 2} \mathscr{E}^{1 / 2} \\
& \lesssim \frac{\varepsilon e^{-\gamma t}}{\delta} \mathscr{E}+\delta \mathscr{D},
\end{aligned}
$$

while for the case that $l=3,4,5$ or 6 ,

$$
\left|\int_{\Omega} \bar{\partial}^{6-l} w \cdot \bar{\partial}^{l} v\left(\bar{\partial}^{6} q+\bar{\partial}^{6} \Psi \cdot v\right)\right| \lesssim\left\|\bar{\partial}^{6-l} w\right\|_{L^{\infty}}\left\|\bar{\partial}^{l} v\right\|_{0}\left\|\bar{\partial}^{6} q+\bar{\partial}^{6} \Psi \cdot v\right\|_{0} \lesssim \varepsilon \mathscr{D},
$$

where we used the Sobolev embedding $H^{1+\delta} \hookrightarrow L^{\infty}$ and Lemma 2.6.

Estimate of $\int_{\Omega} I_{9}$. We see that

$\left|\int_{\Omega} \bar{\partial}^{6} \Psi \cdot v_{t}\left(\bar{\partial}^{6} q+\bar{\partial}^{6} \Psi \cdot v\right)\right| \lesssim\left\|\bar{\partial}^{6} \Psi\right\|_{0}\left\|v_{t}\right\|_{L^{\infty}}\left\|\left(\bar{\partial}^{6} q+\bar{\partial}^{6} \Psi \cdot v\right)\right\|_{0} \lesssim \frac{\mathscr{E}^{1 / 2}}{\chi(t)^{1 / 2}} E_{\beta}^{1 / 2} e^{-\beta t / 2} \mathscr{E} 1 / 2 \lesssim \sqrt{\varepsilon} e^{-\gamma t / 2} \mathscr{E}$

with the decay rate $\gamma>0$ given in Lemma 2.3

Estimate of $\int_{\Omega} \tilde{\mathscr{R}}_{1}$. In the same manner, we find that $\left|\int_{\Omega} \tilde{\mathscr{R}}_{1}\right| \lesssim \varepsilon e^{-\gamma t} \mathscr{E}+\delta \mathscr{D}$. 
Estimate of the boundary integral $\int_{\Gamma} \mathscr{G}_{0}$. We begin with the formula A.10 (whereby we recall (8) $\tilde{n}=A N=\sqrt{R^{2}+R_{\theta}^{2}} n$ ).

$\mathscr{G}_{0}=\underbrace{-\partial_{N} q \bar{\partial}^{6} \Psi \cdot \tilde{n} \bar{\partial}^{6} \Psi \cdot \tilde{n}_{t}}_{K_{1}}-\underbrace{\partial_{N} q \frac{d}{d t}\left[R \bar{\partial}^{6} h\left(-R_{J}+\sum_{a=0}^{5} c_{a}^{J} \bar{\partial}^{a} h \bar{\partial}^{6-a} \xi \cdot\left(h \xi-h_{\theta} T\right)\right)\right]}_{K_{2}}$

A.10

$$
+\underbrace{\partial_{N} q \frac{d}{d t}\left[\left(-R_{J}+\sum_{a=0}^{5} c_{a}^{J} \bar{\partial}^{a} h \bar{\partial}^{6-a \xi} \cdot\left(h \xi-h_{\theta} T\right)\right)^{2}\right]}_{K_{3}}+\underbrace{\sum_{l=1}^{6} a_{l}\left(-\partial_{N} q\right) \bar{\partial}^{6} \Psi \cdot \tilde{n} \bar{\partial}^{6-l}(v-w) \cdot \bar{\partial}^{l} \tilde{n}}_{K_{4}} .
$$

Estimate of $\int_{\Omega} K_{1}$. Note that

$$
\left|\int_{\Gamma} \partial_{N} q_{t} \bar{\partial}^{6} \Psi \cdot \tilde{n} \bar{\partial}^{6} \Psi \cdot \tilde{n}_{t}\right| \lesssim\left|\partial_{N} q_{t}\right|_{L^{\infty}}\left|\tilde{n}_{t}\right|_{L^{\infty}}\left|\bar{\partial}^{6} \Psi\right|_{0}^{2} \lesssim\left|\partial_{N} q_{t}\right|_{1}\left|h_{t}\right|_{2} \frac{\mathscr{E}}{\chi(t)} \lesssim E_{\beta} \frac{e^{-\beta t}}{\chi(t)} \mathscr{E} \lesssim \varepsilon e^{-\gamma t} \mathscr{E}
$$

where we used the trace theorem and Lemma 2.3.

Estimates of $\int_{\Omega} K_{2}$ and $\int_{\Omega} K_{3}$. These two integrals are lower-order and thanks to Lemmas 2.6 and 2.3 are bounded by $\varepsilon e^{-\gamma t} \mathscr{E}+\delta \mathscr{D}$. Note that $|J|=1+O(\varepsilon)$ remains close to 1 due to the a priori smallness bounds from Lemma 2.6

Estimate of $\int_{\Omega} K_{4}$. The estimate of $\int_{\Omega} K_{4}$ requires some explanation, as it has the largest derivative count in $\mathscr{G}_{0}$. In Appendix $\mathrm{A}$, we derive the identity

$$
\bar{\partial}^{6} \Psi \cdot \tilde{n}=R_{J} \bar{\partial}^{6} h-R_{J}+\sum_{a=0}^{5} c_{a}^{J} \bar{\partial}^{a} h \bar{\partial}^{6-a} \xi \cdot\left(h \xi-h_{\theta} \tau\right)
$$

where we recall that $\tau$ is the unit tangent defined by (3) and $R_{J}=R J^{-1}$. Substitution of (77) in the integral $\int_{\Gamma}\left(-\partial_{N} q\right) \bar{\partial}^{6} \Psi \cdot \tilde{n} \bar{\partial}^{6-l}(v-w) \cdot \bar{\partial}^{l} \tilde{n}$ then yields

$\left|\int_{\Gamma}\left(-\partial_{N} q\right) \bar{\partial}^{6} \Psi \cdot \tilde{n} \bar{\partial}^{6-l}(v-w) \cdot \bar{\partial}^{l} \tilde{n}\right| \lesssim\left|\int_{\Gamma}\left(-\partial_{N} q\right) \bar{\partial}^{6-l}(v-w) \cdot \bar{\partial}^{l} \tilde{n}\right|$

$$
+\left|\int_{\Gamma}\left(-\partial_{N} q\right) O\left(\bar{\partial}^{5} h\right) \cdot \tilde{n} \bar{\partial}^{6-l}(v-w) \cdot \bar{\partial}^{l} \tilde{n}\right|+\left|\int_{\Gamma}\left(-\partial_{N} q\right) R \bar{\partial}^{6} h \bar{\partial}^{6-l}(v-w) \cdot \bar{\partial}^{l} \tilde{n}\right| .
$$

The first and the second integrals on the right-hand side of (78) are easily estimated using Hölder's inequality and the Sobolev embedding theorem, while the third integral on the right-hand side of (78) requires some care due to the presence of $\bar{\partial}^{6} h$. If $l=1$ or $l=2$, then

$$
\begin{aligned}
& \left|\int_{\Gamma}\left(-\partial_{N} q\right) R \bar{\partial}^{6} h \bar{\partial}^{6-l}(v-w) \cdot \bar{\partial}^{l} \tilde{n}\right| \leq\left|\sqrt{-\partial_{N} q} \bar{\partial}^{6} h\right|_{0}\left|\sqrt{-\partial_{N} q} R\right|_{L^{\infty}}\left(\left|\bar{\partial}^{4} v\right|_{1}+\left|\bar{\partial}^{4} h_{t}\right|_{1}\right)\left|\bar{\partial}^{l} \tilde{n}\right|_{L^{\infty}} \\
& \lesssim \mathscr{E}^{1 / 2}\|q\|_{2}^{1 / 2}\left(\varepsilon e^{-\gamma t / 2} \mathscr{E}^{1 / 2}+\mathscr{D}^{1 / 2}\right) \varepsilon \lesssim \varepsilon^{2} P\left(\mathscr{E}, E_{\beta}\right) e^{-\gamma t} \mathscr{E}+\delta \mathscr{D}
\end{aligned}
$$

where we have used Corollary 2.11, Lemma 2.6, and then Young's inequality for the last estimate. The case that $l=3,4$, or 5 follows similarly from Lemmas 2.3, 2.6, and 2.10 The case $l=6$ appears problematic because of the term $\bar{\partial}^{6} \tilde{n} \cdot \tau$ which, modulo coefficients, is essentially $\bar{\partial}^{7} h$, one derivative more than appears in $\mathscr{E}$. The integral is, however, easily estimated thanks to the presence of an exact derivative, formed from the integrand $\bar{\partial}^{7} h \bar{\partial}^{6} h$. 
We set $J_{h}=\sqrt{R^{2}+h_{\theta}^{2}}$ and write the unit tangent to $\Gamma(t)$ as $\mathfrak{t}=J_{h}^{-1}\left(R \tau+h_{\theta} N\right)$. A simple computation shows that

$$
n_{\theta}=J_{h}^{-2}\left(R^{2}+2 h_{\theta}^{2}+R h_{\theta \theta}\right) \mathfrak{t} .
$$

Since $v-w=\mathfrak{t} \cdot(v-w) \mathfrak{t}$ on $\Gamma$, we see that $\bar{\partial}^{6} n \cdot(v-w)=\mathfrak{t} \cdot(v-w) \bar{\partial}^{6} n \cdot \mathfrak{t}$. We then write

$$
\bar{\partial}^{6} \tilde{n} \cdot(v-w)=g_{1} \bar{\partial}^{7} h+g_{2},
$$

where $g_{1}=\mathfrak{t} \cdot(v-w) J_{h}^{-2} R$, and where $g_{2}$ is a lower-order term in $v-w$ and has at most six tangential derivatives on $h$. We then write

$$
\begin{aligned}
\int_{\Gamma}\left(-\partial_{N} q\right) R \bar{\partial}^{6} h(v-w) \cdot \bar{\partial}^{6} \tilde{n} & =\int_{\Gamma}\left(-\partial_{N} q\right) R g_{1} \bar{\partial}^{6} h \bar{\partial}^{7} h+\int_{\Gamma}\left(-\partial_{N} q\right) R \bar{\partial}^{6} h g_{2} \\
& =-\frac{1}{2} \int_{\Gamma} \bar{\partial}\left[\left(-\partial_{N} q\right) R g_{1}\right]\left|\bar{\partial}^{6} h\right|^{2}+\int_{\Gamma}\left(-\partial_{N} q\right) R \bar{\partial}^{6} h g_{2}
\end{aligned}
$$

Arguing in a similar fashion as for the case that $l=1$ or 2 , we see that

$$
\left|\int_{\Gamma}\left(-\partial_{N} q\right) R \bar{\partial}^{6} h(v-w) \cdot \bar{\partial}^{6} \tilde{n}\right| \lesssim \sqrt{\varepsilon} e^{-\gamma t} \mathscr{E} .
$$

Step 3. Estimates for $\mathscr{S}_{j}, \tilde{\mathscr{S}}_{j}, \mathscr{H}_{j}$ in (71). We next prove that

$$
\left|\int_{0}^{t} \int_{\Omega}\left(\mathscr{S}_{j}+\tilde{\mathscr{S}}_{j}\right)+\int_{0}^{t} \int_{\Gamma} \mathscr{H}_{j}\right| \leq O(\sqrt{\varepsilon}) \sup _{0 \leq s \leq t} \mathscr{E}(s)+\delta \int_{0}^{t} \mathscr{D}(s), \quad j=1,2,3 .
$$

We will analyze the case that $j=1$, as the estimates for the case that $j=2$ or 3 follow in the same manner. We begin with the definition of $\mathscr{S}_{1}$ given in A.23) as

$$
\begin{gathered}
\mathscr{S}_{1}:=\sum_{\substack{0<a+b<6 \\
a \leq 5, b \leq 1}} c_{a b} \mu \bar{\partial}^{a} \partial_{t}^{b} A_{i}^{k} \bar{\partial}^{5-a} \partial_{t}^{1-b} q_{, k} \bar{\partial}^{5} v^{i}+S_{1}^{\prime} \\
\text { A.23 }-\sum_{l=1}^{5} d_{l} \mu \bar{\partial}^{5-l} \Psi_{t} \cdot \bar{\partial}^{l} v\left(\bar{\partial}^{5} q_{t}+\bar{\partial}^{5} \Psi_{t} \cdot v\right)-\sum_{l=1}^{5} c_{l} \mu \bar{\partial}^{l} A_{i}^{k} \bar{\partial}^{5-l} v_{, k}^{i}\left(\bar{\partial}^{5} q_{t}+\bar{\partial}^{5} \Psi_{t} \cdot v\right),
\end{gathered}
$$

where $S_{1}^{\prime}$ is a lower-order term given by

$$
\begin{aligned}
S_{1}^{\prime}= & \left(\mu q_{, k} A_{i}^{s} A_{r}^{k}\right)_{, s} \bar{\partial}^{5} \Psi_{t}^{r} \bar{\partial}^{5} v^{i}+\left\{\bar{\partial}^{5} \partial_{t}, A_{i}^{k}\right\} q_{, k} \bar{\partial}^{5} v^{i}+\left\{\bar{\partial}^{5} A_{t, i}^{k}\right\} q_{, k} \bar{\partial}^{5} v^{i}+\mu A_{i}^{s} \bar{\partial}^{5} \Psi_{t}^{r} A_{r}^{k} q_{, k}\left\{\bar{\partial}^{5}, \partial_{s}\right\} v^{i} \\
& -\left(\mu A_{i}^{k}\right)_{, k} \bar{\partial}^{5} q_{t} \bar{\partial}^{5} v^{i}+\mu A_{i}^{k} \bar{\partial}^{5} \partial_{t}^{j} q\left\{\bar{\partial}^{5}, \partial_{k}\right\} v^{i}+\mu A_{i}^{k}\left\{\bar{\partial}^{5}, \partial_{k}\right\} \partial_{t} q \bar{\partial}^{5} v^{i}
\end{aligned}
$$

Most of the estimates are completely standard and we focus on the more problematic terms, characterized by the highest number of derivatives applied to two out of the three terms in our cubic integrands. For illustration, in the first term on the right-hand side of A.23 we analyze the cases $(b=0, a=1)$ and $(b=0, a=5)$. If $(b=0, a=1)$ then we first integrate-by-parts and an $L^{\infty}-L^{2}-L^{2}$ Hölder's inequality to find that

$$
\begin{aligned}
\left|\int_{\Omega} \bar{\partial} A_{i}^{k} \bar{\partial}^{4} \partial_{t} q_{, k} \bar{\partial}^{5} v^{i}\right| & =\left|\int_{\Omega} \bar{\partial}^{2} A_{i}^{k} \bar{\partial}^{3} \partial_{t} q_{, k} \bar{\partial}^{5} v^{i}+\int_{\Omega} \bar{\partial} A_{i}^{k} \bar{\partial}^{3} \partial_{t} q_{, k} \bar{\partial}^{6} v^{i}\right| \\
& \leq\left\|\bar{\partial} A_{i}^{k}\right\|_{W^{1, \infty}}\left\|\bar{\partial}^{3} \partial_{t} q_{, k}\right\|_{0}\left(\left\|\bar{\partial}^{5} v^{i}\right\|+\left\|\bar{\partial}^{6} v\right\|_{0}\right) \\
& \leq|h|_{3.5}\left\|q_{t}\right\|_{4}\left(\left\|\bar{\partial}^{5} v^{i}\right\|+\left\|\bar{\partial}^{6} v\right\|_{0}\right) \lesssim \varepsilon \mathscr{D}
\end{aligned}
$$

where Lemmas 2.3 and 2.10 have been used. If $(b=0, a=5)$ then

$$
\left|\int_{\Omega} \bar{\partial}^{5} A_{i}^{k} \partial_{t} q_{, k} \bar{\partial}^{5} v^{i}\right| \leq|h|_{6}\left\|q_{t}\right\|_{2}\left\|\bar{\partial}^{5} v\right\|_{0} \leq \frac{\mathscr{E}^{1 / 2}}{\chi(t)^{1 / 2}} E_{\beta}(t)^{1 / 2} e^{-\beta t / 2} \mathscr{D}^{1 / 2} \lesssim \frac{\varepsilon e^{-\gamma t}}{\delta} \mathscr{E}+\delta \mathscr{D},
$$


where we used Lemmas 2.3, 2.6 and 2.9 The remaining estimates in the expressions A.23 and A.26 for $\mathscr{S}_{1}$ and $\tilde{\mathscr{S}}_{1}$ follow in the identical manner. As to the boundary integral of $\mathscr{H}_{1}$, we state the formula for the integrand derived in A.24 as A.24)

$$
\mathscr{H}_{1}:=2 \partial_{N} q \bar{\partial}^{5} h_{t} R_{J} \sum_{a=0}^{4} \bar{\partial}^{a} h_{t} \bar{\partial}^{5-a} \xi \cdot \tilde{n}+\sum_{l=1}^{4} a_{l}\left(-\partial_{N} q\right) \bar{\partial}^{5} \Psi \cdot \tilde{n} \bar{\partial}^{5} v \cdot \tilde{n} \bar{\partial}^{5-l}(v-w) \cdot \bar{\partial}^{l} \tilde{n} .
$$

We consider the boundary integral of the first term on the right-hand side. We begin with the interpolation bound

$$
\left|h_{t}\right|_{4} \leq\left|h_{t}\right|_{3}^{1 / 2}\left|h_{t}\right|_{5}^{1 / 2} \lesssim \sqrt{\varepsilon} \frac{\mathscr{D}^{1 / 4}}{\chi(t)^{1 / 4}}
$$

where we have used (46) to bound $\left|\bar{\partial}^{3} h_{t}\right|$ and the definition of $\mathscr{D}$ given in (12). If $a=4$ in the first term of the right-hand side of A.24, then

$$
\begin{aligned}
\left|\int_{\Gamma} \partial_{N} q \bar{\partial}^{5} h_{t} R_{J} \bar{\partial}^{4} h_{t} \bar{\partial} \xi \cdot \tilde{n}\right| & =\frac{1}{2}\left|\int_{\Gamma} \partial_{N} q \bar{\partial}\left(\left|\bar{\partial}^{4} h_{t}\right|^{2}\right) R_{J} \bar{\partial} \xi \cdot \tilde{n}\right| \\
& \left.\lesssim\left|\int_{\Gamma} \bar{\partial}\left(\partial_{N} q \bar{\partial} \xi \cdot \tilde{n}\right)\right| \bar{\partial}^{4} h_{t}\right|^{2} \mid \lesssim\|q\|_{4} \varepsilon \frac{\mathscr{D}^{1 / 2}}{\chi(t)^{1 / 2}} \lesssim \varepsilon^{3 / 2} e^{-\gamma t} \mathscr{D}^{1 / 2} \\
& \lesssim \varepsilon^{3 / 2} e^{-2 \gamma t}+\varepsilon^{3 / 2} \mathscr{D},
\end{aligned}
$$

where we have once again used Lemma 2.3 in second inequality, the estimate $[80$, and Young's inequality. If $a \in\{0,1,2,3\}$ then

$$
\begin{aligned}
\left|\int_{\Gamma} \partial_{N} q \bar{\partial}^{5} h_{t} R_{J} \bar{\partial}^{a} h_{t} \bar{\partial}^{5-a} \xi \cdot \tilde{n}\right| & \lesssim\left|\partial_{N} q\right|_{L^{\infty}}\left|\bar{\partial}^{5} h_{t}\right|_{0}\left|R_{J}\right|_{L^{\infty}}\left|\bar{\partial}^{a} h_{t}\right|_{0}\left|\bar{\partial}^{5-a} \xi \cdot \tilde{n}\right|_{L^{\infty}} \\
& \lesssim\|q\|_{3} \frac{\mathscr{D}^{1 / 2}}{\chi(t)^{1 / 2}} \varepsilon \lesssim \varepsilon^{3 / 2} e^{-\gamma t} \mathscr{D}^{1 / 2} \lesssim \varepsilon^{3 / 2} e^{-2 \gamma t}+\varepsilon^{3 / 2} \mathscr{D}
\end{aligned}
$$

where we used Lemmas 2.6 and 2.3 and the same idea as above. The estimates for the second term on the right-hand side of A.24 follow in an analogous vein, relying crucially on Lemmas 2.6 and 2.3. This finishes the proof of (79).

Step 4. The proof of the lemma is a direct consequence of the bounds (74), (76), and (79).

\section{EXISTENCE FOR ALL TIME $t \geq 0$ AND NONLINEAR STABILITY}

4.1. Structure of the proof. The basic goal in our strategy for global-in-time existence and decay of the temperature function is to prove that on any time-interval on which the bootstrap assumptions (29a) and (29b) are valid, we have that

$$
\sup _{0 \leq s \leq t} \mathscr{E}(s)+\int_{0}^{t} \mathscr{D}(s) d s \leq C_{K} \mathscr{E}(0),
$$

where $C_{K}>0$ is some explicit constant depending on $K$. Upon choosing the initial data $\left(q_{0}, h_{0}\right)$ sufficiently small, we can obtain an improvement of the first bootstrap bound in 29a). In Section 4.2 we show the improvement of the bootstrap assumption on $E_{\beta}$ in 29a and in Corollary 2.14 we have already shown the improvement of the bootstrap assumption (29b). By a continuity argument this leads to a global existence result.

In order to implement the above strategy, we start with the basic energy inequality given by (73). Note however the presence of an exponentially growing term $C K^{2} \int_{0}^{t} e^{\eta s} \mathscr{E}(s) d s$ on the right-hand side of $(73)$. That term appears by treating the terms $\int_{\Gamma}\left(-\partial_{N} q_{t}\right) R_{J}^{2}\left|\bar{\partial}^{6-2 j} \partial_{t}^{j} h\right|^{2} d \theta$, 
$j=0,1,2,3$ as error terms. By applying a straightforward Gronwall-type argument, this will be enough to guarantee that solutions to the classical Stefan problem (9) exist on a sufficiently long time-interval $\left[0, T_{K}\right]$, where the time $T_{K}$ may be larger than the time of existence guaranteed by our local well-posedness theorem in [29]. As we explained in the introduction, by a sufficiently long time-interval, we mean a time $T_{K}$ after which the dynamics of the Stefan problem (9a) are, in fact, dominated by the projection of the solution onto the first eigenfunction $\varphi_{1}$ of the Dirichlet-Laplacian.

To prove global existence we need, however, more refined estimates that will show that the $\int_{\Gamma}\left(-\partial_{N} q_{t}\right) R_{J}^{2}\left|\bar{\partial}^{6-2 j} \partial_{t}^{j} h\right|^{2} d \theta$ are in fact sign-definite for $t \geq T_{K}$, leading to the elimination of the exponentially-in-time growing bounds. First, in Section 4.3 we prove strict positivity of the term $\partial_{N} q_{t}$ at time $T_{K}$. Finally in Section 4.4 we use a comparison principle to show that $\partial_{N} q_{t}$ remains positive after time $T_{K}$. This allows us, in turn, to prove the uniform-in-time energy bound and extend the solution for all time $t \geq 0$.

4.2. Boundedness of $E_{\beta}$. The following lemma shows that under the bootstrap assumptions, the bound on $E_{\beta}+\int_{0}^{t} D(s) d s$ from (29b) is improved.

Lemma 4.1. There exists a constant $\tilde{C}$ and $\varepsilon>0$ sufficiently small, such that if the bootstrap assumptions (29a) and (29b) hold with such $\varepsilon$ and $\tilde{C}$, then

$$
E_{\beta}(t)+\int_{0}^{t} D(s) d s<\frac{\tilde{C}}{2} E_{\beta}(0) .
$$

Proof. We set

$x(t)=\|q(t)\|_{4}^{2}+\left\|q_{t}(t)\right\|_{2}^{2}+\left\|q_{t t}(t)\right\|_{0}^{2}$ and recall that $D(t)=\|q(t)\|_{5}^{2}+\left\|q_{t}(t)\right\|_{3}^{2}+\left\|q_{t t}(t)\right\|_{1}^{2}$.

Step 1. Energy inequality for $q_{t t}$. From equation $(\underline{50})$, we see that

$$
\frac{1}{2} \frac{d}{d t}\left\|q_{t t}\right\|_{0}^{2}+\left\|\nabla_{\Psi} q_{t t}\right\|_{0}^{2}=\int_{\Omega} f_{2} q_{t t}
$$

where the forcing term $f_{2}$ is defined just below equation $(50)$. We next show that the righthand side can be bounded by $\varepsilon D$. We first focus on the term $\left(\Psi_{t} \cdot v\right)_{t t}$ in the forcing function $f_{2}$. Using the product rule we obtain

$$
\int_{\Omega}\left(\Psi_{t} \cdot v\right)_{t t} q_{t t}=\underbrace{\int_{\Omega} \Psi_{t t t} \cdot v q_{t t}}_{\mathscr{A}_{1}}+\underbrace{\int_{\Omega} 2 \Psi_{t t} \cdot v_{t} q_{t t}}_{\mathscr{\mathscr { A } _ { 2 }}}+\underbrace{\int_{\Omega} \Psi_{t} \cdot v_{t t} q_{t t}}_{\mathscr{\mathscr { A } _ { 3 }}} .
$$

For the integral $\mathscr{A}_{1}$, we see that

$$
\left|\int_{\Omega} \Psi_{t t t} \cdot v q_{t t}\right| \leq\left\|\Psi_{t t t}\right\|_{0}\|v\|_{L^{\infty}}\left\|q_{t t}\right\|_{0} \lesssim\left|h_{t t t}\right|_{0.5}\|v\|_{2}\left\|q_{t t}\right\|_{0} \lesssim \varepsilon D
$$

where we used the bound (54) to estimate $\left|h_{t t t}\right|_{0.5}$ by $\mathscr{E}^{1 / 2}$. The estimate $\left|\mathscr{A}_{2}\right| \lesssim \varepsilon D$ follows analogously to the estimate for term $\mathscr{A}_{1}$ and the bound on $\mathscr{A}_{3}$ follows from

$$
\left|\mathscr{A}_{3}\right| \lesssim\left\|\Psi_{t}\right\|_{L^{\infty}}\left\|v_{t t}\right\|_{0}\left\|q_{t t}\right\|_{0} \lesssim\left\|\Psi_{t}\right\|_{2} \lesssim \varepsilon D
$$

where we have used Lemma2.6 to infer that $\left\|\Psi_{t}\right\|_{2} \lesssim \varepsilon$. All of the remaining terms in the forcing function $f_{2}$ can be estimated by a straightforward application of the Sobolev embedding theorem together with Lemma 2.6 (to guarantee the smallness of various Sobolev norms applied to the coefficient matrix $\left.\left(A_{i}^{k}\right)_{k, i=1,2}\right)$. Thus, in summary,

$$
\frac{1}{2} \frac{d}{d t}\left\|q_{t t}\right\|_{0}^{2}+\left\|\nabla_{\Psi} q_{t t}\right\|_{0}^{2} \leq C \varepsilon D .
$$


Step 2. Elliptic estimates. We next prove that the quantities $x$ and $y$ are respectively controlled by $\left\|q_{t t}\right\|_{0}^{2}$ and $\left\|\nabla_{\Psi} q_{t t}\right\|_{0}^{2}$. Using the elliptic regularity estimate of Lemma 2.8, the elliptic equations (50), and Lemma 2.6, it follows that

$$
\left\|q_{t}\right\|_{2} \lesssim\left\|q_{t t}\right\|_{0}+\left\|f_{1}\right\|_{0}
$$

and

$$
\|q\|_{4} \lesssim\left\|q_{t}\right\|_{2}+\left\|f_{0}\right\|_{2} \lesssim\left\|q_{t t}\right\|_{0}+\left\|f_{1}\right\|_{0}+\left\|f_{0}\right\|_{2} .
$$

A straightforward application of the Sobolev embedding theorem together with Lemma2.6 implies that

$$
\left\|f_{1}\right\|_{0}^{2}+\left\|f_{0}\right\|_{2}^{2} \lesssim \varepsilon x(t)
$$

Hence, with 82

$$
x(t) \lesssim\left\|q_{t t}\right\|_{0}+\varepsilon x(t)
$$

so that for $\varepsilon>0$ taken sufficiently small,

$$
x(t) \lesssim\left\|q_{t t}(t)\right\|_{0}^{2}
$$

Since $\left\|f_{1}\right\|_{1}^{2}+\left\|f_{0}\right\|_{3}^{2} \lesssim \varepsilon D(t)$, the same argument provides

$$
D \lesssim\left\|q_{t t}\right\|_{1}^{2} \lesssim\left\|\nabla q_{t t}\right\|_{0}^{2} \lesssim\left\|\nabla_{\Psi} q_{t t}\right\|_{0}^{2}
$$

the last inequality following from the uniform lower-bound of the matrix $A A^{T}$.

Step 3. Poincaré inequality. The following bound holds:

$$
\left(\lambda_{1}-O(\varepsilon)\right)\|f\|_{0}^{2} \leq\left\|\nabla_{\Psi} f\right\|_{0}^{2},
$$

where $\nabla_{\Psi}=A^{T} \nabla$ and $f \in H_{0}^{1}(\Omega)$. To see (86), note that the inequalities (49) and (42) show that

$$
\|A-\mathrm{Id}\|_{L^{\infty}} \lesssim \varepsilon
$$

from which it follows that $A_{i}^{k} A_{i}^{j} \xi_{k} \xi_{j} \geq(1-O(\varepsilon))|\xi|^{2}$ for all $\xi \in \mathbb{R}^{2}$. The Poincaré inequality $\lambda_{1}\|f\|_{0}^{2} \leq\|\nabla f\|_{0}^{2}$ for all $q \in H_{0}^{1}(\Omega)$ then concludes the proof.

Step 4. The differential inequality and decay. From (81) and (85) we obtain that

$$
\frac{1}{2} \frac{d}{d t}\left\|q_{t t}\right\|_{0}^{2}+(1-O(\varepsilon))\left\|\nabla_{\Psi} q_{t t}\right\|_{0}^{2} \leq 0
$$

Using the Poincaré inequality [86, it follows that

$$
\frac{d}{d t}\left\|q_{t t}\right\|_{0}^{2}+\left(2 \lambda_{1}-O(\varepsilon)\right)\left\|q_{t t}\right\|_{0}^{2} \leq 0
$$

From this differential inequality, we immediately infer the bound

$$
\left\|q_{t t}(t)\right\|_{0}^{2} \leq\left\|q_{t t}(0)\right\| e^{-\left(2 \lambda_{1}-O(\varepsilon)\right) t} .
$$

From the elliptic estimate in Step 2, it finally follows that

$$
x(t) \leq C\left\|q_{t t}(0)\right\|_{0}^{2} e^{-\left(2 \lambda_{1}-O(\varepsilon)\right) t} \leq C^{\prime} E_{\beta}(0) e^{-\left(2 \lambda_{1}-O(\varepsilon)\right) t} .
$$

Since $E_{\beta}(t)=x(t) e^{\beta t}$ and $\beta=2 \lambda_{1}-\eta<2 \lambda_{1}-O(\varepsilon)$ for $\varepsilon$ sufficiently small, it is now clear that we can choose $\tilde{C}$ so that on the time interval of validity of bootstrap assumptions $29 \mathrm{a}$ and $29 \mathrm{~b}$ we actually have the improved bound $E_{\beta}(t) \leq \frac{\tilde{C}}{2} e^{-\beta t}$. 


\subsection{Pointwise positivity of $\partial_{N} q_{t}$ at time $T_{K}=\bar{C} \ln K$.}

Lemma 4.2. Assume that the solution $(q, h)$ to the Stefan problem (9) exists on a given time interval $[0, T]$. Let the bootstrap assumptions (29a) and (29b) hold on that time interval with $\varepsilon>0$ sufficiently small, and assume the smallness assumption (22) for the initial data. There exists a universal constant $\bar{C}$ such that if $T \geq T_{K}:=\bar{C} \ln K$, then

$$
-q_{t}\left(T_{K}, x\right)>C c_{1} e^{-\lambda_{1} T_{K}} \varphi_{1}(x), \quad x \in B_{1}(0),
$$

where $\varphi_{1}$ is the first eigenfunction of the Dirichlet-Laplacian on $\Omega$ and $c_{1}=\int_{\Omega} q_{0} \varphi_{1} d x$. As a consequence,

$$
\inf _{x \in \Gamma} \partial_{N} q_{t}\left(T_{K}, x\right)>0 .
$$

Proof. Step 1. Hardy-type estimate. As a consequence of the higher-order Hardy inequality (see Lemma 1 in [18]) and the Sobolev embedding theorem, for any $f \in H^{2.25}\left(B_{1}(0)\right) \cap$ $H_{0}^{1}\left(B_{1}(0)\right)$,

$$
\sup _{x \in B_{1}(0)}\left|\frac{f(x)}{\varphi_{1}(x)}\right| \leq C\|f\|_{2.25}
$$

where $\varphi_{1}$ is the first eigenfunction of the Dirichlet-Laplacian on the unit ball.

Step 2. The Duhamel formula. Let

$$
q_{0}=\sum_{j=1}^{\infty} c_{j} \varphi_{j}
$$

be the eigenvector decomposition of the initial datum $q_{0}$ with respect to the $L^{2}$ orthonormal basis $\left\{\varphi_{1}, \varphi_{2}, \ldots\right\}$ associated with the Dirichlet-Laplacian on the unit disk $B_{1}(0)$. Writing the time-differentiated Stefan problem as a perturbation of the linear heat equation, we see that in $\Omega, q_{t}$ satisfies

$$
q_{t t}-\Delta q_{t}=N(q, h)
$$

where

$$
N(q, h):=\left(a_{i j}-\delta_{i j}\right) q_{t, i j}+b_{i} q_{t, i}+a_{i j, t} q_{, i j}+b_{i, t} q_{, i}+A_{, i t}^{k} q_{, k} w^{i}+A_{i}^{k} q_{, k} w_{t}^{i},
$$

and the coefficients $a_{i j}, b_{i}$ are defined in (70). Note that at time $t=0, q_{t}(0)=\Delta q_{0}+\nabla q_{0}$. $w_{0}$; moreover, since $\Delta \varphi_{j}=-\lambda_{j} \varphi_{j}$ and $e^{t \Delta}$ is a linear semi-group, the Duhamel principle implies that the solution $q_{t}$ to $(88)$ can be written as

$$
-q_{t}=\underbrace{c_{1} \lambda_{1} e^{-\lambda_{1} t} \varphi_{1}+\sum_{j=2}^{\infty} c_{j} \lambda_{j} e^{-\lambda_{j} t} \varphi_{j}}_{=: X}-\underbrace{e^{t \Delta}\left(\nabla q_{0} \cdot w_{0}\right)}_{=: Y}-\underbrace{\int_{0}^{t} e^{(t-s) \Delta} N(q, h)}_{=: Z} .
$$

We first prove that $X(t)>0$ for times $t=\bar{C} \ln K$, where $\bar{C}$ denotes a universal constant. We shall then show that at time $t=\bar{C} \ln K,|Y(t)|+|Z(t)|$ is bounded by a small fraction of $X(t)$.

Step 3. Estimate of $X$. We begin by writing $X$ as

$$
X(t, x)=c_{1} \lambda_{1} e^{-\lambda_{1} t} \varphi_{1}(x)+c_{1} \lambda_{1} e^{-\lambda_{1} t} \varphi_{1}(x)\left(\sum_{j=2}^{\infty} \frac{c_{j} \lambda_{j}}{c_{1} \lambda_{1}} e^{\left(\lambda_{1}-\lambda_{j}\right) t} \frac{\varphi_{j}(x)}{\varphi_{1}(x)}\right) .
$$


Our goal is to prove that the term

$$
\sigma:=\sum_{j=2}^{\infty} \frac{c_{j} \lambda_{j}}{c_{1} \lambda_{1}} e^{\left(\lambda_{1}-\lambda_{j}\right) t} \frac{\varphi_{j}(x)}{\varphi_{1}(x)}
$$

is small. By Corollary 2.2

$$
\frac{\left|c_{j}\right|}{c_{1}} \leq K \text { for all integers } j \geq 2 .
$$

Furthermore, using the normalization $\left\|\varphi_{j}\right\|_{0}=1$, and the eigenvalue problem, $\Delta \varphi_{j}=$ $-\lambda_{j} \varphi_{j}$, elliptic regularity shows that $\|\varphi\|_{2} \leq \lambda_{j}$ and that $\|\varphi\|_{4} \leq \lambda_{j}^{2}$; hence, linear interpolation provides us with the inequality

$$
\left\|\varphi_{j}\right\|_{2.25} \lesssim \lambda_{j}^{1.25}
$$

Using (92) and (93), together with the bound (87), we see that

$$
|\sigma| \leq C K \sum_{j=2}^{\infty} \lambda_{j}^{2.25} e^{\left(\lambda_{1}-\lambda_{j}\right) t}
$$

Since $\lambda_{1}<\lambda_{2} \leq \lambda_{3} \leq \ldots$, there exists a constant $c^{*}$, uniform in $j \geq 2$, such that $\lambda_{1} / \lambda_{j}<$ $\left(1-2 c^{*}\right)$. This implies that

$$
\left(\lambda_{1}-\lambda_{j}\right)<-2 c^{*} \lambda_{j} \text { for integers } j \geq 2 .
$$

In particular, for $t \geq \bar{C} \ln K$

$$
C K \lambda_{j}^{2.25} e^{-c^{*} \lambda_{j} t} \leq C K \lambda_{j}^{2.25} K^{-\bar{C} c^{*} \lambda_{j}}=C \frac{\lambda_{j}^{2.25}}{K^{\bar{C} c^{*} \lambda_{j}-1}}<\frac{1}{2}
$$

for $\bar{C}$ chosen sufficiently large, but independent of $K$. (Recall that $K>1$ since $K \geq \frac{\left\|q_{0}\right\|_{1}}{\left\|q_{0}\right\|_{0}} \geq$ $1+\lambda_{1}>6$.) Hence, from (91) and the previous inequality it follows

$$
|\sigma| \leq \frac{1}{2} \sum_{j=2}^{\infty} e^{-c^{*} \lambda_{j} t} \leq \frac{1}{2} \sum_{j=2}^{\infty} K^{-\bar{C} c^{*} \lambda_{j}}<\frac{1}{2} .
$$

Plugging this into 90 , we obtain for any $x \in B_{1}(0)$

$$
X(t, x) \geq \frac{1}{2} c_{1} \lambda_{1} e^{-\lambda_{1} t} \varphi_{1}(x)>0, t \geq \bar{C} \ln K .
$$

Step 4. Estimates of $Y$ and $Z$. The term $Y$ satisfies the estimate

$$
\|Y(t, x)\|_{L^{\infty}} \lesssim\left\|e^{t \Delta}\left(\nabla q_{0} \cdot w_{0}\right)\right\|_{2} \lesssim e^{-\lambda_{1} t}\left\|\nabla q_{0} \cdot w_{0}\right\|_{2} \lesssim e^{-\lambda_{1} t}\left\|q_{0}\right\|_{3}\left\|w_{0}\right\|_{2} \lesssim \varepsilon c_{1} e^{-\lambda_{1} t},
$$

where we used the Sobolev embedding theorem together with the bound $\left\|q_{0}\right\|_{3} \lesssim K c_{1}$, which follows from $\left\|q_{0}\right\|_{4} /\left\|q_{0}\right\|_{0} \leq K$. Thus $|Y(t, x)|<\frac{1}{4}|X(t, x)|$ with $\varepsilon$ sufficiently small. Next, to estimate $Z$ which vanishes at the boundary, we have that

$$
\begin{aligned}
\frac{|Z|}{\varphi_{1}(x)} & \leq \int_{0}^{t}\left|\frac{e^{\Delta(t-s)} N(q, h)(s)}{\varphi_{1}(x)}\right| d s \lesssim \int_{0}^{t}\left\|e^{\Delta(t-s)} N(q, h)(s)\right\|_{2.25} d s \\
& \lesssim \int_{0}^{t}\|N(q, h)(s)\|_{2.25} d s \lesssim \sqrt{t}\left(\int_{0}^{t}\|N(q, h)(s)\|_{2.25}^{2} d s\right)^{1 / 2} .
\end{aligned}
$$

In the above chain of inequalities, we have used the bound (87) for the second inequality, and the fact that $\left\|e^{t \Delta}\right\|_{H^{s} \rightarrow H^{s}} \leq 1$.

We shall conclude our estimate by showing that

$$
\int_{0}^{t}\|N(q, h)(s)\|_{2.25}^{2} d s \lesssim c_{1}^{11 / 5} \varepsilon^{9 / 5} .
$$


We recall that

$$
N(q, h):=\underbrace{\left(a_{i j}-\delta_{i j}\right) q_{t, i j}}_{=: Z_{1}}+\underbrace{b_{i} q_{t, i}+a_{i j, t} q_{, i j}+b_{i, t} q_{, i}+A_{, i t}^{k} q_{, k} w^{i}+A_{i}^{k} q_{, k} w_{t}^{i}}_{=: Z_{2}},
$$

and note that $Z_{1}$ is the highest-order term with respect to the number of derivatives applied to $q$. Writing $Z_{1}=(a-\mathrm{Id}) D^{2} q_{t}$, where Id denotes the identity matrix, we see that

$$
\int_{0}^{t}\left\|Z_{1}\right\|_{2.25}^{2} d s \lesssim\|a-\operatorname{Id}\|_{2.25}^{2}\left\|D^{2} q_{t}\right\|_{2.25}^{2} \lesssim \sup _{0 \leq s \leq t}\|a-\operatorname{Id}\|_{2.25}^{2} \int_{0}^{t}\left\|q_{t}\right\|_{4.25}^{2} .
$$

From the sharp estimate [40, we infer that $\sup _{0 \leq s \leq t}\|a-\mathrm{Id}\|_{2.25}^{2} \lesssim c_{1}^{2}$; furthermore, for the term $\left\|q_{t}\right\|_{4.25}$ we apply the interpolation estimate $\left\|q_{t}\right\|_{4.25}^{2} \lesssim\left\|q_{t}\right\|_{2}^{1 / 5}\left\|q_{t}\right\|_{4.5}^{9 / 5} \lesssim c_{1}^{1 / 5} e^{-\beta t / 10}\left\|q_{t}\right\|_{4.5}^{9 / 5}$.

Using Lemma 2.10, we then infer that

$$
\int_{0}^{t}\left\|Z_{1}\right\|_{2.25}^{2} d s \lesssim c_{1}^{11 / 5} \int_{0}^{t} e^{-\beta t / 10}\left\|q_{t}\right\|_{4.5}^{9 / 5} \lesssim c_{1}^{11 / 5} \varepsilon^{9 / 5},
$$

the last inequality following from Hölder's inequality and the fact that $\int_{0}^{t}\left\|q_{t}\right\|_{4.5}^{2} \lesssim \varepsilon^{2}$ by Lemma 2.10 and the bootstrap assumption 29a.

Analogous estimates are applied to the term $Z_{2}$ to finally deduce (95). By (95) and the above chain of estimates, it follows that

$$
\frac{|Z|}{\varphi_{1}(x)} \lesssim \sqrt{t} c_{1}^{11 / 10} \varepsilon^{9 / 10}
$$

Hence, at time $T=\bar{C} \ln K$

$$
\begin{aligned}
|Z(T, x)| & \lesssim \bar{C}^{1 / 2} \ln K^{1 / 2} c_{1} c_{1}^{1 / 10} \varepsilon^{9 / 10} \varphi_{1}(x) \lesssim c_{1} \frac{\varepsilon_{0}^{1 / 10}}{F(K)^{1 / 20}} \bar{C}^{1 / 2} \ln K^{1 / 2} \varepsilon^{9 / 10} \varphi_{1}(x) \\
& \leq c_{1} \frac{\varepsilon}{F(K)^{1 / 20}} \bar{C}^{1 / 2} \ln K^{1 / 2} \varphi_{1}(x)<\frac{1}{4} c_{1} \lambda_{1} e^{-\lambda_{1} T} \varphi_{1}(x) \leq \frac{1}{2} X(t, x) .
\end{aligned}
$$

Note that we have used the estimate $c_{1}^{1 / 10} \lesssim \varepsilon_{0}^{1 / 10} / F(K)^{1 / 20}$ (which follows from $\left\|q_{0}\right\| \lesssim$ $\mathscr{E}(0)^{1 / 2}$ and the smallness assumption (22) ) as well as $\varepsilon_{0} \leq \varepsilon$ which is going to hold by our choice of $\varepsilon_{0}$. Observe that the very last inequality follows from (94). The next-to-last bound is equivalent to

$$
\frac{\varepsilon}{F(K)^{1 / 20}}<\frac{\lambda_{1}}{4 \bar{C}^{1 / 2} \ln K^{1 / 2} K^{\bar{C} \lambda_{1}}},
$$

which then follows from the choice (23) of the function $F(K)$ in Remark 1.3 The second inequality above follows from the estimate $c_{1} \lesssim\left\|q_{0}\right\| \lesssim \mathscr{E}(0)^{1 / 2} \lesssim \varepsilon / F(K)^{1 / 2}$.

Step 5. Finishing the proof. From the above estimates on $X, Y$, and $Z$ it finally follows that for any $x \in B_{1}(0), T=\bar{C} \ln K$,

$$
\begin{aligned}
-q_{t}(T, x) & \geq|X(T, x)|-|Y(T, x)|-|Z(t, x)| \geq X(T, x)-\frac{1}{2} X(T, x)-\frac{1}{4} X(T, x)=\frac{1}{4} X(t, x) \\
& \geq c^{*} c_{1} e^{-\lambda_{1} T} \varphi_{1}(x) .
\end{aligned}
$$

Finally, since $\partial_{N} \varphi_{1} \geq c$ for some $c>0$ uniformly over $\Gamma$ and since $\varphi_{1}>0$ in $\Omega$, it follows $\inf _{x \in \Gamma} \partial_{N} q_{t}(T, x)>0$. 
4.4. Proof of Theorem 1.2. Step 1. By Proposition 3.4 with $\varepsilon$ sufficiently small, we conclude that

$$
\sup _{0 \leq t \leq T} \mathscr{E}(t)+\int_{0}^{T} \mathscr{D}(t) d t \leq 2 \mathscr{E}(0)+C K^{2} \int_{0}^{T} e^{\eta t} \mathscr{E}(t) d t, \quad t \in[0, \mathscr{T}],
$$

where $\mathscr{T}$ is the maximal interval of existence on which the bootstrap assumptions 29a) and (29b) hold (with $\varepsilon$ sufficiently small). Our goal is to prove that on $[0, \mathscr{T}]$, the quantity $\mathscr{E}(t)$ is bounded from above by $2 \mathscr{E}(0) e^{2 C K^{2} t}$. We shall accomplish that by bounding $\mathscr{E}(t)$ from above by the function $g(t): \mathbb{R}^{+} \rightarrow \mathbb{R}$, which is defined as the solution of the differential equation

$$
g^{\prime}(t)=C K^{2} e^{\eta t} g(t), \quad g(0)=2 \mathscr{E}(0) .
$$

Solving this differential equation, we obtain

$$
\begin{aligned}
g(t) & =2 \mathscr{E}(0) e^{\frac{C K^{2}}{\eta}\left(e^{\eta t}-1\right)}=2 \mathscr{E}(0) e^{\left(1+\sum_{k=2}^{\infty} \eta^{k-1} t^{k} / k !\right) C K^{2} t} \\
& =2 \mathscr{E}(0) e^{(1+O(\eta)) C K^{2} t} \leq 2 \mathscr{E}(0) e^{2 C K^{2} t},
\end{aligned}
$$

where the convergence of the sum $\sum_{k=2}^{\infty} \eta^{k-1} t^{k} / k$ ! is guaranteed for times $t \leq \frac{1}{\sqrt{\eta}}$. Applying the integral Gronwall inequality to the difference $\mathscr{E}(t)-g(t)$, it follows from (96) and the previous inequality that

$$
\mathscr{E}(t) \leq g(t) \leq 2 \mathscr{E}(0) e^{2 C K^{2} t}
$$

for any $t \leq \mathscr{T}$. Our goal is to prove that $\mathscr{T} \geq \bar{C} \ln K$. Using (96) once again, we obtain the same smallness bound on $\int_{0}^{t} \mathscr{D}(s) d s$ to finally conclude that

$$
\sup _{0 \leq s \leq t} \mathscr{E}(s)+\int_{0}^{t} \mathscr{D}(s) d s \leq 2 \mathscr{E}(0) e^{2 C K^{2} t} .
$$

For $t \leq \bar{C} \ln K$, 971) and smallness assumption (22) on $\mathscr{E}(0)$ implies that

$$
\sup _{0 \leq s \leq t} \mathscr{E}(s)+\int_{0}^{t} \mathscr{D}(s) d s \leq \varepsilon / 2 .
$$

Moreover, by Lemma 4.1 and since the bootstrap assumptions (29a and 29b are valid on $[0, \mathscr{T}]$ it follows that

$$
E_{\beta}(t)+\int_{0}^{t} D(s) d s<\frac{\tilde{C}}{2} E_{\beta}(0) .
$$

Thus, by the continuity of $\mathscr{E}+E_{\beta}$ and the maximality of $\mathscr{T}$, we conclude $\min \{\mathscr{T}, \bar{C} \ln K\}=$ $\bar{C} \ln K=T_{K}$ since the bootstrap assumptions are still satisfied at time $t=\bar{C} \ln K$ (the argument is true as long as $\eta$ above is chosen in such a way that $\frac{1}{\sqrt{\eta}}>\bar{C} \ln K$ ). By the local well-posedness theorem from [29] and the continuity of $\mathscr{E}$ and $E_{\beta}$ in time, we actually have the strict inequality $\mathscr{T}>T_{K}$ as we can extend the solution locally in time. We will argue by contradiction that $\mathscr{T}=\infty$. Assume $\mathscr{T}<\infty$.

Step 2. Preserving the positivity of $\partial_{N} q_{t}$. We next show that $\partial_{N} q_{t}>0$ on the time interval $\left[T_{K}, \mathscr{T}[\right.$. This will be done with help of Lemma 4.2 and the maximum principle. We start by constructing a suitable comparison function,

$$
P(t, r)=\kappa_{1} e^{-\frac{3}{2} \lambda_{1} t}\left(\varphi_{1}(r)-\kappa_{2}\left(1-r^{2}\right)\right),
$$


with positive constants $\kappa_{1}, \kappa_{2}$ to be specified later. A straightforward calculation shows that

$$
\begin{aligned}
\left(\partial_{t}-a_{i j} \partial_{i j}-b_{i} \partial_{i}\right) P= & \kappa_{1} e^{-\frac{3}{2} \lambda_{1} t}\left[-\frac{1}{2} \lambda_{1} \varphi_{1}-2 \kappa_{2} \operatorname{Tr}(a)\right. \\
& \left.+\frac{3}{2} \lambda_{1} \kappa_{2}\left(1-r^{2}\right)-\left(a_{i j}-\delta_{i j}\right) \varphi_{1}-b \cdot\left(\nabla \varphi_{1}+2 \kappa_{2} x\right)\right] .
\end{aligned}
$$

Observe that both $\varphi_{1}$ and $\left(1-r^{2}\right)$ vanish for $r=1$, the trace of the matrix $a$ is very close to 2, i.e., $a_{11}+a_{22}=2+O(\varepsilon)$ and the coefficients $b_{i}$ are very small, i.e. $|b|=O(\varepsilon)$. Note that the first and the second term in the parenthesis on the right-hand side of 99) are negative, while the fourth and the fifth term are small of order $\varepsilon$. If $r=|x|$ is close to 1 , then the second term dominates the third term and if $r$ is away from the boundary $r=1$, then one can choose $\kappa_{2}>0$ so that the first term dominates the third term. It follows easily that there exists a $\kappa_{2}>0$ and some constant $C_{1}$ such that

$$
\left(\partial_{t}-a_{i j} \partial_{i j}-b_{i} \partial_{i}\right) P<-C_{1} \kappa_{1} e^{-\frac{3}{2} \lambda_{1} t} .
$$

It then follows from (100) and (70) that

$\left(\partial_{t}-a_{i j} \partial_{i j}-b_{i} \partial_{i}\right)\left(-q_{t}-P\right)>-\left(\partial_{t} a_{i j} q,{ }_{i j}+\partial_{t} b_{i} q_{i}+\partial_{t} A_{, i}^{k} q_{, k} w^{i}+A_{i}^{k} q_{, k} w_{t}^{i}\right)+C_{1} \kappa_{1} e^{-\frac{3}{2} \lambda_{1} t}$.

Note, however, that the term in parenthesis on the right-hand side above is a quadratic non-linearity and as such decays at least as fast as $e^{-2 \beta t}$ :

$$
\begin{aligned}
& \left\|\partial_{t} a_{i j} q_{, i j}+\partial_{t} b_{i} q_{i}+\partial_{t} A_{, i}^{k} q_{, k} w^{i}+A_{i}^{k} q_{, k} w_{t}^{i}\right\|_{L^{\infty}} \\
& \leq\left\|\partial_{t} a_{i j} q,_{i j}\right\|_{1+\delta}+\left\|\partial_{t} b_{i} q_{i}\right\|_{1+\delta}+\left\|\partial_{t} A_{, i}^{k} q_{, k} w^{i}\right\|_{1+\delta}+\left\|A_{i}^{k} q_{, k} w_{t}^{i}\right\|_{1+\delta} \\
& \leq C_{2} E_{\beta}(0)^{1 / 2} \varepsilon e^{-2 \beta t} \leq C_{2} c_{1} \varepsilon e^{-2 \beta t} .
\end{aligned}
$$

Now, using 101 and the above bound, we note that by choosing the constant $\kappa_{1}:=\frac{C_{2}}{C_{1}} c_{1} \varepsilon$, we have that

$$
\left(\partial_{t}-a_{i j} \partial_{i j}-b_{i} \partial_{i}\right)\left(-q_{t}-P\right)>C_{2} c_{1} \varepsilon e^{-\frac{3}{2} \lambda_{1} t}-C_{2} c_{1} \varepsilon e^{-2 \beta t}>0,
$$

since $2 \beta=2 \lambda_{1}-\eta>\frac{3}{2} \lambda_{1}$. The previous bound implies that $-q_{t}-P$ is a supersolution for the operator $\partial_{t}-a_{i j} \partial_{i j}-b_{i} \partial_{i}$. Moreover, by the construction of $P$, we have $-q_{t}-P=0$ on $\Gamma=\partial B_{1}(0)$. Furthermore, at time $T_{K}=\bar{C} \ln K$, we have by Lemma 4.2 and 98, that

$$
\left.\left(-q_{t}-P\right)\right|_{T=\bar{C} \ln K}>C c_{1} e^{-\lambda_{1} T} \varphi_{1}(x)-C c_{1} \varepsilon e^{-\frac{3}{2} \lambda_{1} T} \varphi_{1}(x)+C c_{1} \varepsilon \kappa_{2} e^{-\frac{3}{2} \lambda_{1} T}\left(1-r^{2}\right)>0
$$

for $\varepsilon$ sufficiently small. Thus, by Lemma2.12, there exists a constant $m>0$ such that

$$
-q_{t}-P \geq m(1-r) e^{-\left(\lambda_{1}-O(\varepsilon)\right) t}, t>T_{K},
$$

or in other words

$$
\begin{aligned}
-q_{t} & \geq m(1-r) e^{-\left(\lambda_{1}-O(\varepsilon)\right) t}+C c_{1} \varepsilon(1-r) e^{-\frac{3}{2} \lambda_{1} t}\left(\frac{\varphi_{1}(r)}{1-r}-\kappa_{2}(1+r)\right) \\
& =(1-r) e^{-\left(\lambda_{1}-O(\varepsilon)\right) t}\left(m+C c_{1} \varepsilon e^{\left(-\frac{1}{2} \lambda_{1} t-O(\varepsilon)\right) t}\left(\frac{\varphi_{1}(r)}{1-r}-\kappa_{2}(1+r)\right)\right),
\end{aligned}
$$

which readily gives the positivity of $\partial_{N} q_{t}$ on the time-interval $\left[T_{K}, \mathscr{T}\left[\right.\right.$ since $\frac{\varphi_{1}(r)}{1-r}-\kappa_{2}(1+$ $r)>0$ by our choice of $\kappa_{2}$ above. We conclude that the positivity of $-q_{t}$ at time $T_{K}=\bar{C} \ln K$ is a property preserved by our bootstrap regime and moreover we get a quantitative lower bound on $\partial_{N} q_{t}$ on the time interval $\left[T_{K}, \mathscr{T}[\right.$. 
Step 3. Conclusion. Thus for any $t \in\left[T_{K}, \mathscr{T}[\right.$, the energy identity takes the form

$$
\begin{aligned}
& \mathscr{E}(t)+\int_{T_{K}}^{t} \mathscr{D}(t)+\frac{1}{2} \sum_{j=0}^{3} \int_{T_{K}}^{t} \int_{\Gamma} \partial_{N} q_{t} R_{J}^{2}\left|\bar{\partial}^{6-2 j} \partial_{t}^{j} h\right|^{2} d x \\
& =\mathscr{E}\left(T_{K}\right)+\sum_{i=1}^{4} \int_{T_{K}}^{t} \int_{\Omega}\left\{\mathscr{R}_{i}+\mathscr{S}_{i}\right\}+\sum_{i=0}^{4} \int_{T_{K}}^{t} \int_{\Omega}\left\{\tilde{\mathscr{R}}_{i}+\tilde{\mathscr{S}}_{i}\right\}+\sum_{i=0}^{4} \int_{T_{K}}^{t} \int_{\Gamma}\left\{\mathscr{G}_{i}+\mathscr{H}_{i}\right\},
\end{aligned}
$$

where we formally define $\mathscr{S}_{4}=\tilde{\mathscr{S}}_{4}=\mathscr{G}_{4}=0$. In particular, by the energy estimates stated in (76) and (79) the right-hand side of the above identity is bounded by

$$
\mathscr{E}\left(T_{K}\right)+O(\sqrt{\varepsilon}) \sup _{T_{K} \leq s \leq t} \mathscr{E}(s)+(O(\varepsilon)+\delta) \int_{T_{K}}^{t} \mathscr{D}(s) d s .
$$

Note here the absence of the exponentially growing term in the above bound as opposed to their presence in Proposition 3.4 This is due to the fact that terms $\int_{T_{K}}^{t} \int_{\Gamma} \partial_{N} q_{t} R_{J}^{2}\left|\bar{\partial}^{6-2 j} \partial_{t}^{j} h\right|^{2} d x$, $j=0,1,2,3$ are positive and no longer treated as error terms. By absorbing the small multiples of $\sup _{T_{K} \leq s \leq t} \mathscr{E}(s)$ and $\int_{T_{K}}^{t} \mathscr{D}(s) d s$ into the left-hand side and using the positivity of $\partial_{N} q_{t}$ from Step 2, we obtain

$$
\sup _{T_{K} \leq s \leq t} \mathscr{E}(s)+\int_{T_{K}}^{t} \mathscr{D}(s) d s \leq 2 \mathscr{E}\left(T_{K}\right) \leq 8 \mathscr{E}(0) e^{2 C K^{2} T_{K}}
$$

by (97). Finally, we choose $\varepsilon_{0}$ in the statement of Theorem 1.2 so that $\varepsilon_{0}^{2}<\varepsilon^{2} / 2$. Bound (103) and the condition $\mathscr{E}(0) \lesssim \varepsilon_{0}^{2} / F(K)$ (with $F(K)$ given as in (23) ) imply

$$
\sup _{T_{K} \leq s \leq t} \mathscr{E}(s)+\int_{T_{K}}^{t} \mathscr{D}(s) d s \leq \frac{\varepsilon^{2}}{2}
$$

Together with Lemma 4.1 and Corollary 2.14, we infer that the bootstrap assumptions 29a and $29 \mathrm{~b}$ are improved. Since $\mathscr{E}$ is continuous in time, we can extend the solution by the local well-posedness theory to an interval $\left[0, \mathscr{T}+T^{*}\right]$ for some small positive time $T^{*}$. This however contradicts the maximality of $\mathscr{T}$ and hence $\mathscr{T}=\infty$. This concludes the proof of the main theorem.

\section{THE $d$-DIMENSIONAL CASE ON GENERAL NEAR-SPHERICAL DOMAINS}

In this section we briefly sketch the set-up of the problem in general dimensions and explain how to adapt the arguments from the 2-D case to the 3-D case. Let $\Omega(t) \subset \mathbb{R}^{d}$ be an open simply connected subset of $\mathbb{R}^{d}, d \geq 2$. The moving boundary $\Gamma(t)=\partial \Omega(t)$ is parametrized as a graph over the unit sphere $\mathbb{S}^{\bar{d}-1}$

$$
\Gamma(t)=\left\{x \mid x=R(t, \xi) \xi=(1+h(t, \xi)) \xi, \xi \in \mathbb{S}^{d-1}\right\} .
$$

Initially $R_{0}(\xi)$ is assumed to be close to 1, i.e. $R_{0}(\xi)-1=h_{0}(\xi)=O(\varepsilon)$. We shall assume that $\Omega_{0}$ is diffeomorphic to $B_{1}(0)$, where $\Phi: \Omega \rightarrow \Omega_{0}$ is the diffeomorphism mapping of the unit ball onto the initial domain. Moreover, let $\tilde{\Psi}$ denote the family of diffeomorphisms from the initial domain $\Omega_{0}$ to the moving domain $\Omega(t)$, satisfying the harmonic equation $\Delta \tilde{\Psi}=0$ and the boundary condition $\tilde{\Psi}\left(\Gamma_{0}\right)=\Gamma(t)$. We shall pull back the Stefan problem onto the unit ball $B_{1}(0)$ via the map $\Psi: B_{1}(0) \rightarrow \Omega(t)$ given as a composition of $\tilde{\Psi}$ and $\Phi$ :

$$
\Psi=\tilde{\Psi} \circ \Phi .
$$


Upon defining $q, v, w$, and $A$ just as in Section (9), the Stefan problem (1) takes exactly the same form as 9 . Abusing the notation, the normal velocity $\mathscr{V}(\Gamma(t))$ is now given by

$$
\mathscr{V}(\Gamma(t))=\frac{R_{t} R}{\sqrt{R^{2}+|\nabla R|_{\mathbb{S}^{d-1}}^{2}} .}
$$

Here $|\nabla R|_{\mathbb{S}^{d-1}}^{2}$ stands for the squared norm of the Riemannian gradient of $R(t, \cdot)$ on the unit sphere $\mathbb{S}^{d-1}$, which is a coordinate invariant expression. The gauge equation for $\Psi$ transforms into

$$
\Delta_{\Phi^{-1}} \Psi=0, \quad \Psi\left(t, \mathbb{S}^{d-1}\right)=\Gamma(t)
$$

due to the assumption $\Delta \tilde{\Psi}=0$ and the definition of $\Psi$. This easily implies the optimal trace bound $\|\Psi\|_{H^{s}(\Omega)} \lesssim|\Psi|_{H^{s-0.5}}(\Omega)$ due to the the smoothness of $\Phi$ and the closeness assumption $\|D \Phi-\mathrm{Id}\|_{H^{s}} \lesssim \varepsilon$, with $s$ sufficiently large. When $d=3$, the Sobolev embedding theorem requires us to raise the degree of spatial regularity in the definition our energy spaces by one derivative.

The second key observation is that the lower bound for the quantity $\chi(t)$ is obtained in the same way as in the case that $d=2$, from Lemma 2.12. We $v_{1}$ denote the first eigenvalue of the operator $-\Delta_{\Phi^{-1}}$, which is the pull-back of the negative Laplacian from the initial domain $\Omega_{0}$ to $B_{1}(0)$. By Lemma2.12 we obtain that

$$
\chi(t) \gtrsim c_{1} e^{-\lambda t},
$$

where $\left|\lambda-v_{1}\right| \leq O\left(\left|h-h_{0}\right|_{W^{2, \infty}}+\left|h_{t}\right|_{L^{\infty}}\right)=O(\varepsilon)$. Since $\|D \Phi-\operatorname{Id}\|_{H^{s}} \lesssim \varepsilon$ for $s$ sufficiently large, we have $\left|v_{1}-\lambda_{1}\right| \lesssim \varepsilon$, where we recall that $\lambda_{1}$ stands for the first eigenvalue of the Dirichlet-Laplacian. Together, the two previous estimates imply the analogous conclusion of Corollary 2.14, namely

$$
\chi(t) \gtrsim c_{1} e^{-\lambda_{1}-\tilde{\lambda}_{1}(t) t}, \quad \tilde{\lambda}_{1}=O(\varepsilon) .
$$

Let $\bar{\partial}^{i}$ denote the tangential component of $\partial^{i}$ restricted to $\mathbb{S}^{2}$. To each multi-index $\vec{\alpha}=$ $\left(\alpha_{1}, \alpha_{2}, \alpha_{3}\right)$ we associate the tangential operator $\bar{\partial}^{\vec{\alpha}}=\bar{\partial}^{\alpha_{1}} \bar{\partial}^{\alpha_{2}} \bar{\partial}^{\alpha_{3}}$. With $d=3$, we define

$\mathscr{E}_{3 D}(t)=\mathscr{E}_{3 D}(q, h)(t):=$

$\frac{1}{2} \sum_{|\alpha|+2 b \leq 6}\left\|\mu^{1 / 2} \bar{\partial} \vec{\alpha} \partial_{t}^{b} v\right\|_{L_{x}^{2}}^{2}+\frac{1}{2} \sum_{|\vec{\alpha}|+2 b \leq 7}\left|\left(-\partial_{N} q\right)^{1 / 2} R_{J} \bar{\partial} \vec{\alpha} \partial_{t}^{b} h\right|_{L_{x}^{2}}^{2}+\frac{1}{2} \sum_{|\vec{\alpha}|+2 b \leq 7}\left\|\mu^{1 / 2}\left(\bar{\partial} \vec{\alpha} \partial_{t}^{b} q+\bar{\partial}^{\vec{\alpha}} \partial_{t}^{b} \Psi \cdot v\right)\right\|_{L_{x}^{2}}^{2}$

$\sum_{|\vec{\alpha}|+2 b \leq 6}\left\|(1-\mu)^{1 / 2} \partial_{\vec{\alpha}} \partial_{t}^{b} v\right\|_{L_{x}^{2}}^{2}+\frac{1}{2} \sum_{|\vec{\alpha}|+2 b \leq 7}\left\|(1-\mu)^{1 / 2}\left(\partial_{\vec{\alpha}} \partial_{t}^{b} q+\partial_{\vec{\alpha}} \partial_{t}^{b} \Psi \cdot v\right)\right\|_{L_{x}^{2}}^{2}$

and

$$
\begin{aligned}
& \mathscr{D}_{3 D}(t)=\mathscr{D}_{3 D}(q, h)(t):= \\
& \quad \sum_{|\vec{\alpha}|+2 b \leq 7}\left\|\mu^{1 / 2} \bar{\partial}^{\vec{\alpha}} \partial_{t}^{b} v\right\|_{L_{x}^{2}}^{2}+\sum_{|\vec{\alpha}|+2 b \leq 6}\left|\left(-\partial_{N} q\right)^{1 / 2} R_{J} \bar{\partial}^{\vec{\alpha}} \partial_{t}^{b} h_{t}\right|_{L_{x}^{2}}^{2}+\sum_{|\vec{\alpha}|+2 b \leq 6}\left\|\mu^{1 / 2}\left(\bar{\partial}^{\vec{\alpha}} \partial_{t}^{b} q_{t}+\bar{\partial}^{\vec{\alpha}} \partial_{t}^{b} \Psi_{t} \cdot v\right)\right\|_{L_{x}^{2}}^{2} \\
& +\sum_{|\vec{\alpha}|+2 b \leq 7}\left\|(1-\mu)^{1 / 2} \partial_{\vec{\alpha}} \partial_{t}^{b} v\right\|_{L_{x}^{2}}^{2}+\sum_{|\vec{\alpha}|+2 b \leq 6}\left\|(1-\mu)^{1 / 2}\left(\partial_{\vec{\alpha}} \partial_{t}^{b} q_{t}+\partial_{\vec{\alpha}} \partial_{t}^{b} \Psi_{t} \cdot v\right)\right\|_{L_{x}^{2}}^{2} .
\end{aligned}
$$

The lemmas of Section 2 carry through analogously, as do the energy estimates of Section 3 By the continuity argument of Section 4 we arrive at the 3-D version of our main theorem: 
Theorem 5.1 (The 3-D case). Let $\left(q_{0}, h_{0}\right)$ satisfy the Taylor sign condition (18), the strict positivity assumption (17), and the corresponding compatibility conditions. Let $\left\|q_{0}\right\|_{4} /\left\|q_{0}\right\|_{0} \leq K$. Then there exists an $\varepsilon_{0}=\varepsilon_{0}(K)>0$ and $\delta_{0}>0$ such that if $\mathscr{E}\left(q_{0}, h_{0}\right)<$ $\varepsilon_{0}^{2}$, then there exists a unique global solution to problem (9), satisfying

$$
\sup _{0 \leq t \leq \infty} \mathscr{E}_{3 D}(q(t), h(t))<C \delta_{0}^{2},
$$

for some universal constant $C>0$, and $\|q\|_{H^{5}\left(B_{1}(0)\right)}^{2} \leq C e^{-\beta t}$, where $\beta=2 \lambda_{1}-C \varepsilon_{0}$ and $\lambda_{1}$ is the smallest eigenvalue of the Dirichlet-Laplacian on the unit ball $B(0,1) \subset \mathbb{R}^{3}$. The moving boundary $\Gamma(t)$ settles asymptotically to some nearby steady surface $\bar{\Gamma}$ and we have uniform-in-time estimate

$$
\sup _{0 \leq t<\infty}\left|h-h_{0}\right|_{5.5} \lesssim \sqrt{\delta_{0}}
$$

\section{Appendix A. Proof of Proposition 3.1}

To prove the energy identity of Proposition 3.1 we start by applying the differential operator of the form $\bar{\partial}^{6-2 j} \partial_{t}^{j}$ to the equation (9b). For $j=0,1,2,3$ we multiply it then by $\bar{\partial}^{6-2 j} \partial_{t}^{j}$ and integrate-by-parts. Additionally, if $j=1,2,3$ we apply the operator $\bar{\partial}^{7-2 j} \partial_{t}^{j}$ to $\left[9 \mathrm{~b}\right.$, multiply by $\bar{\partial}^{7-2 j} \partial_{t}^{j-1} v^{i}$, and again integrate-by-parts.

Based on these two cases we distinguish between the two different types of identities.

A.1. Identities of the first type. Recall that $\mu: \bar{\Omega} \rightarrow \mathbb{R}$ is a $C^{\infty}$ cut-off function with the property

$$
\mu(x) \equiv 0 \quad \text { if }|x| \leq 1 / 2 ; \quad \mu(x) \equiv 1 \text { if } 3 / 4 \leq|x| \leq 1 .
$$

Applying the tangential differential operator $\mu \bar{\partial}^{6}$ to the equation $9 \mathrm{gb}$, , multiplying it by $\bar{\partial}^{6} v^{i}$ and integrating over $\Omega$, we obtain

$$
\left(\mu \bar{\partial}^{6} v^{i}+\mu \bar{\partial}^{6} A_{i}^{k} q_{, k}+\mu A_{i}^{k} \bar{\partial}^{6} q_{, k}, \bar{\partial}^{6} v^{i}\right)_{L^{2}}=\sum_{l=1}^{5} c_{l}\left(\mu \bar{\partial}^{l} A_{i}^{k} \bar{\partial}^{6-l} q_{, k}, \bar{\partial}^{6} v^{i}\right)_{L^{2}},
$$

where $c_{l}=\left(\begin{array}{l}6 \\ l\end{array}\right)$. Recalling [47], we write

$$
\bar{\partial}^{6} A_{i}^{k}=-A_{i}^{s} \bar{\partial}^{6} \Psi_{, s}^{r} A_{r}^{k}+\left\{\bar{\partial}^{6}, A_{i}^{k}\right\}
$$

where $\left\{\bar{\partial}^{6}, A_{i}^{k}\right\}$ denotes the lower-order commutator defined in $\left.477 \mathrm{~b}\right)$. With this identity, we obtain

$$
\begin{aligned}
\left(\mu \bar{\partial}^{6} A_{i}^{k} q_{, k}, \bar{\partial}^{6} v^{i}\right)_{L^{2}(\Omega)} & =-\left(\mu A_{i}^{s} \bar{\partial}^{6} \Psi_{, s}^{r} A_{r}^{k} q_{, k}, \bar{\partial}^{6} v^{i}\right)_{L^{2}(\Omega)}+\left(\mu\left\{\bar{\partial}^{6}, A_{i}^{k}\right\} q_{, k}, \bar{\partial}^{6} v^{i}\right)_{L^{2}(\Omega)} \\
& =-\int_{\Gamma} q_{, k} A_{i}^{s} \bar{\partial}^{6} \Psi^{r} A_{r}^{k} \bar{\partial}^{6} v^{i} N^{s}+\int_{\Omega} \mu A_{i}^{s} \bar{\partial}^{6} \Psi^{r} A_{r}^{k} q_{, k} \bar{\partial}^{6} v_{, s}^{i}+\int_{\Omega} \mathscr{T}_{1} \\
& =-\int_{\Gamma} q_{, k} A_{i}^{s} \bar{\partial}^{6} \Psi^{r} A_{r}^{k} \bar{\partial}^{6} v^{i} N^{s}-\int_{\Omega} \mu A_{i}^{s} \bar{\partial}^{6} \Psi^{r} v^{r} \bar{\partial}^{6} v_{, s}^{i}+\int_{\Omega} \mathscr{T}_{1},
\end{aligned}
$$

where we have integrated-by-parts with respect to $x^{s}$ for the second equality, and have used the identity $v^{r}=-A_{r}^{k} q_{, k}$ for the last equality; the error term $\mathscr{T}_{1}$ is given by

$$
\mathscr{T}_{1}=\left(\mu q_{, k} A_{i}^{s} A_{r}^{k}\right),_{s} \bar{\partial}^{6} \Psi_{K}^{r} \bar{\partial}^{6} v^{i}+\mu\left\{\bar{\partial}^{6}, A_{i}^{k}\right\} q_{, k}, \bar{\partial}^{6} v^{i}+\mu A_{i}^{s} \bar{\partial}^{6} \Psi^{r} A_{r}^{k} q_{, k}\left\{\bar{\partial}^{6}, \partial_{s}\right\} v^{i} .
$$

Furthermore, integration-by-parts with respect to $x^{k}$ yields

$$
\begin{aligned}
& \left(\mu A_{i}^{k} \bar{\partial}^{6} q_{, k}, \bar{\partial}^{6} v^{i}\right)_{L^{2}}=\int_{\Omega} \mu A_{i}^{k} \partial_{k} \bar{\partial}^{6} q \bar{\partial}^{6} v^{i}+\int_{\Omega} \mu A_{i}^{k}\left\{\bar{\partial}^{6}, \partial_{k}\right\} q \bar{\partial}^{6} v^{i} \\
& =-\int_{\Omega} \mu A_{i}^{k} \bar{\partial}^{6} q \bar{\partial}^{6} v_{, k}^{i}-\int_{\Omega}\left(\mu A_{i}^{k}\right)_{, k} \bar{\partial}^{6} q^{6} \bar{\partial}^{6}+\int_{\Omega} \mu A_{i}^{k}\left\{\bar{\partial}^{6}, \partial_{k}\right\} q \bar{\partial}^{6} v^{i},
\end{aligned}
$$


where we have used $\bar{\partial}^{6} q=0$ on $\Gamma$, and where $\left\{\bar{\partial}^{6}, \partial_{k}\right\}$ denotes the lower-order commutator. Summing A.11 and A.2, we find that

(A.3)

$$
\begin{aligned}
& \left(\mu \bar{\partial}^{6} A_{i}^{k} q_{, k}+\mu A_{i}^{k} \bar{\partial}^{6} q_{, k}, \bar{\partial}^{6} v^{i}\right)_{L^{2}(\Omega)}=-\int_{\Gamma} q_{, k} A_{i}^{s} \bar{\partial}^{6} \Psi^{r} A_{r}^{k} \bar{\partial}^{6} v^{i} N^{s} \\
& -\int_{\Omega} \mu A_{i}^{k} \bar{\partial}^{6} v_{, k}^{i}\left(\bar{\partial}^{6} q+\bar{\partial}^{6} \Psi \cdot v\right)+\int_{\Omega}\left(\mathscr{T}_{1}-\left(\mu A_{i}^{k}\right)_{, k} \bar{\partial}^{6} q^{6} \bar{\partial}^{6} v^{i}+\mu A_{i}^{k}\left\{\bar{\partial}^{6}, \partial_{k}\right\} q \bar{\partial}^{6} v^{i}\right) .
\end{aligned}
$$

The first two terms on the right-hand side of A.3 will be the source of positive definite quadratic contributions to the energy. To extract the quadratic coercive contribution from the first integral on the right-hand side of (A.3), note that $q_{, k}=N^{k} \partial_{N} q$ on $\Gamma$, and also recall from 8 , the normal vector $\tilde{n}=A^{T} N$. Thus

$$
-\int_{\Gamma} q_{, k} A_{i}^{s} \bar{\partial}^{6} \Psi^{r} A_{r}^{k} \bar{\partial}^{6} v^{i} N^{s}=\int_{\Gamma}\left(-\partial_{N} q\right) \bar{\partial}^{6} \Psi^{r} \tilde{n}^{r} \bar{\partial}^{6} v^{i} \tilde{n}^{i}=\int_{\Gamma}\left(-\partial_{N} q\right) \bar{\partial}^{6} \Psi \cdot \tilde{n} \bar{\partial}^{6} v \cdot \tilde{n}
$$

Using the boundary condition (10), we reexpress $\bar{\partial}^{6} v \cdot \tilde{n}$ as

$$
\begin{aligned}
\bar{\partial}^{6} v \cdot \tilde{n} & =\bar{\partial}^{6} w \cdot \tilde{n}+\bar{\partial}^{6}(v-w) \cdot \tilde{n} \\
& =\bar{\partial}^{6} w \cdot \tilde{n}+\bar{\partial}^{6}(\underbrace{(v-w) \cdot \tilde{n}}_{=0})-\sum_{l=1}^{6} a_{l} \bar{\partial}^{6-l}(v-w) \cdot \bar{\partial}^{l} \tilde{n} \\
& =\bar{\partial}^{6} \Psi_{t} \cdot \tilde{n}-\sum_{l=1}^{6} a_{l} \bar{\partial}^{6-l}(v-w) \cdot \bar{\partial}^{l} \tilde{n} .
\end{aligned}
$$

Due to the above identity and A.4, we obtain that

$$
\int_{\Gamma}\left(-\partial_{N} q\right) \bar{\partial}^{6} \Psi \cdot \tilde{n} \bar{\partial}^{6} v \cdot \tilde{n}=\int_{\Gamma}\left(-\partial_{N} q\right) \bar{\partial}^{6} \Psi \cdot \tilde{n} \bar{\partial}^{6} \Psi_{t} \cdot \tilde{n}-\sum_{l=1}^{6} a_{l} \int_{\Gamma}\left(-\partial_{N} q\right) \bar{\partial}^{6} \Psi \cdot \tilde{n} \bar{\partial}^{6-l}(v-w) \cdot \bar{\partial} l \tilde{n}
$$

$$
=\int_{\Gamma}\left(-\partial_{N} q\right) \frac{1}{2} \frac{d}{d t}\left|\bar{\partial}^{6} \Psi \cdot \tilde{n}\right|^{2} d x^{\prime}+\int_{\Gamma} \partial_{N} q \bar{\partial}^{6} \Psi \cdot \tilde{n} \bar{\partial}^{6} \Psi \cdot \tilde{n}_{t}-\sum_{l=1}^{6} a_{l} \int_{\Gamma}\left(-\partial_{N} q\right) \bar{\partial}^{6} \Psi \cdot \tilde{n} \bar{\partial}^{6-l}(v-w) \cdot \bar{\partial} l \tilde{n} .
$$

Recall that $\tilde{n}=J^{-1}\left(R N-R_{\theta} \tau\right)=J^{-1}\left(N+h N-h_{\theta} \tau\right)$. Thus, using $\Psi(t, \xi)=N+h(t, \xi) N$, we obtain via the Leibniz rule

$$
\begin{aligned}
\bar{\partial}^{6} \Psi \cdot \tilde{n} & =\left[\bar{\partial}^{6} N+\bar{\partial}^{6}(h N)\right] \cdot\left[(1+h) N-h_{\theta} \tau\right] J^{-1} \\
& =\left(-R+R \bar{\partial}^{6} h+\sum_{a=0}^{5} c_{a} \bar{\partial}^{a} h \bar{\partial}^{6-a} N \cdot\left(h N-h_{\theta} \tau\right)\right) J^{-1} \\
& =\left(-R_{J}+R_{J} \bar{\partial}^{6} h+\sum_{a=0}^{5} c_{a}^{J} \bar{\partial}^{a} h \bar{\partial}^{6-a} N \cdot\left(h N-h_{\theta} \tau\right)\right),
\end{aligned}
$$

where we have used the relations $\bar{\partial}^{2} N=-N$ and $N \cdot \tau=0$ and also denoted $c_{a}^{J}=c_{a} J^{-1}$ (recall $\left.R_{J}=R J^{-1}\right)$. From here we obtain

(A.6)

$$
\begin{aligned}
& \frac{1}{2} \frac{d}{d t}\left(\left|\bar{\partial}^{6} \Psi \cdot \tilde{n}\right|^{2}\right)=\frac{1}{2} \frac{d}{d t}\left(R_{J}^{2}\left|\overline{\partial^{6}} h\right|^{2}\right) \\
& +\frac{d}{d t}\left[\left(-R_{J}+2 R_{J} \bar{\partial}^{6} h+\sum_{a=0}^{5} c_{a}^{J} \bar{\partial}^{a} h \bar{\partial}^{6-a} N \cdot\left(h N-h_{\theta} \tau\right)\right)\left(-R_{J}+\sum_{a=0}^{5} c_{a}^{J} \bar{\partial}^{a} h \bar{\partial}^{6-a} N \cdot\left(h N-h_{\theta} \tau\right)\right)\right] .
\end{aligned}
$$


Thus, going back to A.5, we obtain

$$
\int_{\Gamma}\left(-\partial_{N} q\right) \bar{\partial}^{6} \Psi \cdot \tilde{n} \bar{\partial}^{6} \Psi_{t} \cdot \tilde{n}=\frac{1}{2} \frac{d}{d t} \int_{\Gamma}\left(-\partial_{N} q\right) R_{J}^{2}\left|\bar{\partial}^{6} h\right|^{2}+\frac{1}{2} \int_{\Gamma} \partial_{N} q_{t} R_{J}^{2}\left|\bar{\partial}^{6} h\right|^{2}+\int_{\Gamma} \mathscr{T}_{2},
$$

where the error term $\mathscr{T}_{2}$ is given by

$$
\mathscr{T}_{2}=\left(-\partial_{N} q\right) \frac{d}{d t}\left[\left(-R_{J}+2 R_{J} \bar{\partial}^{6} h+\sum_{a=0}^{5} c_{a}^{J} \bar{\partial}^{a} h \bar{\partial}^{6-a} N \cdot\left(h N-h_{\theta} \tau\right)\right)\left(-R_{J}+\sum_{a=0}^{5} c_{a}^{J} \bar{\partial}^{a} h \bar{\partial}^{6-a} N \cdot\left(h N-h_{\theta} \tau\right)\right)\right] .
$$

As to the second term on the right-hand side of A.3, note that

$$
A_{i}^{k} \bar{\partial}^{6} v_{, k}^{i}=\bar{\partial}^{6}\left(A_{i}^{k} v_{, k}^{i}\right)-\sum_{l=1}^{6} c_{l} \bar{\partial}^{l} A_{i}^{k} \bar{\partial}^{6-l} v_{, k}^{i}=-\bar{\partial}^{6}\left(q_{t}+v \cdot w\right)-\sum_{l=1}^{6} c_{l} \bar{\partial}^{l} A_{i}^{k} \bar{\partial}^{6-l} v_{, k}^{i}
$$

where $A_{i}^{k} v_{, k}^{i}=-\operatorname{div} \Psi v=-\left(q_{t}+v \cdot w\right)$ by the parabolic equation 9a $)$. Thus

$$
\begin{aligned}
& -\int_{\Omega} \mu A_{i}^{k} \bar{\partial}^{6} v_{, k}^{i}\left(\bar{\partial}^{6} q+\bar{\partial}^{6} \Psi \cdot v\right)=\int_{\Omega} \mu \bar{\partial}^{6}\left(q_{t}+\Psi_{t} \cdot v\right)\left(\bar{\partial}^{6} q+\bar{\partial}^{6} \Psi \cdot v\right)+\sum_{l=1}^{6} c_{l} \int_{\Omega} \mu \bar{\partial}^{l} A_{i}^{k} \bar{\partial}^{6-l} v_{, k}^{i}\left(\bar{\partial}^{6} q+\bar{\partial}^{6} \Psi \cdot v\right) \\
& =\frac{1}{2} \frac{d}{d t} \int_{\Omega} \mu\left(\bar{\partial}^{6} q+\bar{\partial}^{6} \Psi \cdot v\right)^{2}+\int_{\Omega} \mu\left(\sum_{l=1}^{6} d_{l} \bar{\partial}^{6-l} \Psi_{t} \cdot \bar{\partial}^{l} v-\bar{\partial}^{6} \Psi \cdot v_{t}\right)\left(\bar{\partial}^{6} q+\bar{\partial}^{6} \Psi \cdot v\right)
\end{aligned}
$$

$$
+\sum_{l=1}^{6} c_{l} \int_{\Omega} \mu \bar{\partial}^{l} A_{i}^{k} \bar{\partial}^{6-l} v_{, k}^{i}\left(\bar{\partial}^{6} q+\bar{\partial}^{6} \Psi \cdot v\right)
$$

Combining A.3 - A.7 we obtain

$$
\begin{aligned}
& \int_{\Omega} \mu\left|\bar{\partial}^{6} v\right|^{2} d x+\frac{1}{2} \frac{d}{d t} \int_{\Gamma}\left(-\partial_{N} q\right) R_{J}^{2}\left|\bar{\partial}^{6} h\right|^{2} d x^{\prime}+\frac{1}{2} \frac{d}{d t} \int_{\Omega} \mu\left(\bar{\partial}^{6} q+\bar{\partial}^{6} \Psi \cdot v\right)^{2} d x \\
& =-\frac{1}{2} \frac{d}{d t} \int_{\Gamma}\left(-\partial_{N} q\right)\left|\sum_{a=0} c_{a} \bar{\partial}^{a} R \bar{\partial}^{6-a} N \cdot \tilde{n}\right|^{2} d x^{\prime}+\int_{\Omega} \mathscr{R}_{0}+\int_{\Gamma} \mathscr{G}_{0}
\end{aligned}
$$

with the error terms $\mathscr{R}_{0}$ and $\mathscr{G}_{0}$ given by

$$
\begin{aligned}
\mathscr{R}_{0}= & \mu \sum_{l=1}^{5} c_{l} \bar{\partial}^{l} A_{i}^{k} \bar{\partial}^{6-l} q_{, k} \bar{\partial}^{6} v^{i}+\left(\mu q_{, k} A_{i}^{s} A_{r}^{k}\right)_{, s} \bar{\partial}^{6} \Psi^{r} \bar{\partial}^{6} v^{i}+\mu\left\{\bar{\partial}^{6}, A_{i}^{k}\right\} q_{, k} \bar{\partial}^{6} v^{i} \\
& +\mu A_{i}^{s} \bar{\partial}^{6} \Psi^{r} A_{r}^{k} q_{, k}\left\{\bar{\partial}^{6}, \partial_{s}\right\} v^{i}-\left(\mu A_{i}^{k}\right)_{, k} \bar{\partial}^{6} q^{6} \bar{\partial}^{6} v^{i}
\end{aligned}
$$

$$
-\mu A_{i}^{k}\left\{\bar{\partial}^{6}, \partial_{k}\right\} q \bar{\partial}^{6} v^{i}-\mu \sum_{l=1}^{6}\left(c_{l} \bar{\partial}^{l} A_{i}^{k} \bar{\partial}^{6-l} v_{, k}^{i}+d_{l} \bar{\partial}^{6-l} w \cdot \bar{\partial}^{l} v-\bar{\partial}^{6} \Psi \cdot v_{t}\right)\left(\bar{\partial}^{6} q+\bar{\partial}^{6} \Psi \cdot v\right) ;
$$

$$
\begin{aligned}
& \mathscr{G}_{0}=-\partial_{N} q \bar{\partial}^{6} \Psi \cdot \tilde{n} \bar{\partial}^{6} \Psi \cdot \tilde{n}_{t}+\left(-\partial_{N} q\right) \frac{d}{d t}\left[R_{J} \bar{\partial}^{6} h\left(-R_{J}+\sum_{a=0}^{5} c_{a}^{J} \bar{\partial}^{a} h \bar{\partial}^{6-a} N \cdot\left(h N-h_{\theta} \tau\right)\right)\right] \\
& -\partial_{N} q \frac{d}{d t}\left[\left(-R_{J}+\sum_{a=0}^{5} c_{a}^{J} \bar{\partial}^{a} h \bar{\partial}^{6-a} N \cdot\left(h N-h_{\theta} \tau\right)\right)^{2}\right]+\sum_{l=1}^{6} a_{l}\left(-\partial_{N} q\right) \bar{\partial}^{6} \Psi \cdot \tilde{n} \bar{\partial}^{6-l}(v-w) \cdot \bar{\partial}^{l} \tilde{n} .
\end{aligned}
$$

Let now $\alpha=\left(\alpha_{1}, \alpha_{2}\right)$ be an arbitrary multi-index of order 6 . Applying the operator (1$\mu) \partial^{\alpha}$ to 9 b and multiplying by $\partial^{\alpha} v^{i}$, we obtain

$$
\begin{aligned}
& \left((1-\mu) \partial^{\alpha} v^{i}+(1-\mu) \partial^{\alpha} A_{i}^{k} q_{, k}+(1-\mu) A_{i}^{k} \partial^{\alpha} q_{, k}, \partial^{\alpha} v^{i}\right)_{L^{2}(\Omega)} \\
& =-\sum_{0<\beta \leq \alpha} c_{\beta}\left((1-\mu) \partial^{\beta} A_{i}^{k} \partial^{\alpha-\beta} q_{, k}, \partial^{\alpha} v^{i}\right)_{L^{2}}
\end{aligned}
$$


In the same way as above we arrive at the following energy identity

$$
\int_{\Omega}(1-\mu)\left|\partial^{\alpha} v\right|^{2} d x+\frac{1}{2} \frac{d}{d t} \int_{\Omega}(1-\mu)\left(\partial^{\alpha} q_{t}+\partial^{\alpha} \Psi \cdot v\right)^{2} d x=\int_{\Omega} \tilde{\mathscr{R}}_{0} d x,
$$

where

(A.12)

$$
\begin{aligned}
& \tilde{\mathscr{R}}_{0}=(1-\mu) \sum_{0<\beta<\alpha} c_{\beta} \partial^{\beta} A_{i}^{k} \partial^{\alpha-\beta} q_{, k} \partial^{\alpha} v^{i}+(1-\mu)\left(\left(q_{, k} A_{i}^{s} A_{r}^{k}\right),{ }_{s} \partial^{\alpha} \Psi^{r} \partial^{\alpha} v^{i}+\left\{\partial^{\alpha}, A_{i}^{k}\right\} q_{, k} \partial^{\alpha} v^{i}\right) \\
& -(1-\mu) \sum_{0<\beta \leq \alpha}\left(c_{\beta} \partial^{\beta} A_{i}^{k} \partial^{\alpha-\beta} v_{, k}^{i}+d_{\beta} \partial^{\alpha-\beta} w \cdot \partial^{\beta} v-\partial^{\alpha} \Psi \cdot v_{t}\right)\left(\partial^{\alpha} q+\partial^{\alpha} \Psi \cdot v\right),
\end{aligned}
$$

Summing the identities A.8 and A.11, with $j=0$ we arrive at

$$
\begin{aligned}
& \int_{\Omega} \mu\left|\bar{\partial}^{6-2 j} \partial_{t}^{j} v\right|^{2}+\sum_{|\alpha|=6-2 j} \int_{\Omega}(1-\mu)\left|\partial^{\alpha} \partial_{t}^{j} v\right|^{2}+\frac{1}{2} \frac{d}{d t} \int_{\Gamma}\left(-\partial_{N} q\right) R_{J}^{2}\left|\bar{\partial}^{6-2 j} \partial_{t}^{j} h\right|^{2} \\
& +\frac{1}{2} \frac{d}{d t} \int_{\Omega} \mu\left(\bar{\partial}^{6-2 j} \partial_{t}^{j} q+\bar{\partial}^{6-2 j} \partial_{t}^{j} \Psi \cdot v\right)^{2}+\frac{1}{2} \frac{d}{d t} \sum_{|\alpha|=6-2 j} \int_{\Omega}(1-\mu)\left(\partial^{\alpha} \partial_{t}^{j} q+\partial^{\alpha} \partial_{t}^{j} \Psi \cdot v\right)^{2}
\end{aligned}
$$

$$
=-\int_{\Gamma}\left(-\partial_{N} q_{t}\right) R_{J}^{2}\left|\bar{\partial}^{6-2 j} \partial_{t}^{j} h\right|^{2}+\int_{\Omega}\left(\mathscr{R}_{j}+\tilde{\mathscr{R}}_{j}\right)+\int_{\Gamma} \mathscr{G}_{j},
$$

By imitating the same calculation as above we obtain the remaining error terms. With $j=1,2,3$ the formulas for $\mathscr{R}_{j}, \tilde{\mathscr{R}}_{j}$, and $\mathscr{G}_{j}$ in A.13 read

$$
\begin{aligned}
\mathscr{R}_{j}= & \sum_{0<a+b<6-j} \mu d_{a b} \bar{\partial}^{a} \partial_{t}^{b} A_{i}^{k} \bar{\partial}^{6-2 j-a} \partial_{t}^{j-b} q_{, k} \bar{\partial}^{6-2 j} \partial_{t}^{j} v^{i}+\left(\mu q_{, k} A_{i}^{s} A_{r}^{k}\right)_{, s} \bar{\partial}^{6-2 j} \partial_{t}^{j} \Psi^{r} \bar{\partial}^{6-2 j} \partial_{t}^{j} v^{i} \\
& +\mu A_{i}^{s} \bar{\partial}^{6-2 j} \partial_{t}^{j} \Psi^{r} A_{r}^{k} q,_{k}\left\{\bar{\partial}^{6-2 j}, \partial_{s}\right\} \partial_{t}^{j} v^{i}+\mu\left\{\bar{\partial}^{6-2 j} \partial_{t}^{j}, A_{i}^{k}\right\} q_{, k} \bar{\partial}^{6-2 j} \partial_{t}^{j} v^{i} \\
& -\left(\mu A_{i}^{k}\right)_{, k} \bar{\partial}^{6-2 j} \partial_{t}^{j} q^{6-2 j} \partial_{t}^{j} v^{i}-\mu A_{i}^{k}\left\{\bar{\partial}^{6-2 j}, \partial_{k}\right\} \partial_{t}^{j} q^{6-2 j} \bar{\partial}_{t}^{j} v^{i} \\
& -\mu \sum_{0 \leq a+b<6-j}\left(d_{a b} \bar{\partial}^{6-2 j-a} \partial_{t}^{j-b} A_{i}^{k} \bar{\partial}^{a} \partial_{t}^{b} v_{, k}^{i}+d_{a b} \bar{\partial}^{a} \partial_{t}^{b} \Psi_{t} \cdot \bar{\partial}^{6-2 j-a} \partial_{t}^{j-b} v-\bar{\partial}^{6-2 j} \partial_{t}^{j} \Psi \cdot v_{t}\right)
\end{aligned}
$$

$$
\times\left(\bar{\partial}^{6-2 j} \partial_{t}^{j} q+\bar{\partial}^{6-2 j} \partial_{t}^{j} \Psi \cdot v\right)
$$

$\mathscr{G}_{j}=-\partial_{N} q \bar{\partial}^{6-2 j} \partial_{t}^{j} \Psi \cdot \tilde{n} \bar{\partial}^{6-2 j} \partial_{t}^{j} \Psi \cdot \tilde{n}_{t}+\partial_{N} q \partial_{t}\left[\bar{\partial}^{6-2 j} \partial_{t}^{j} h R_{J}\left(-R_{J}+\sum_{a=0}^{5-2 j} d_{a}^{J} \bar{\partial}^{a} \partial_{t}^{j} h \bar{\partial}^{6-2 j-a} N \cdot \tilde{n}\right)\right]$

$$
+\frac{d}{d t}\left[\left(\sum_{a=0}^{5-2 j} d_{a}^{J} \bar{\partial}^{a} \partial_{t}^{j} h \bar{\partial}^{6-2 j-a} N \cdot \tilde{n}\right)^{2}\right]
$$

$$
\begin{gathered}
+\sum_{0 \leq a+b<6-j} d_{a b}\left(-\partial_{N} q\right) \bar{\partial}^{6-2 j} \partial_{t}^{j} \Psi \cdot \tilde{n} \bar{\partial}^{6-2 j-a} \partial_{t}^{j-b}(v-w) \cdot \bar{\partial}^{a} \partial_{t}^{b} \tilde{n} . \\
\tilde{\mathscr{R}}_{j}=(1-\mu) \sum_{0<\beta<\alpha} c_{\beta} \partial^{\beta} A_{i}^{k} \partial^{\alpha-\beta} q_{, k} \partial^{\alpha} v^{i}+(1-\mu) \tilde{\mathscr{T}}_{1}
\end{gathered}
$$

$$
-(1-\mu) \sum_{0<\beta \leq \alpha}\left(c_{\beta} \partial^{\beta} A_{i}^{k} \partial^{\alpha-\beta} v_{, k}^{i}+(1-\mu) d_{\beta} \partial^{\alpha-\beta} w \cdot \partial^{\beta} v-\partial^{\alpha} \Psi \cdot v_{t}\right)\left(\partial^{\alpha} q+\partial^{\alpha} \Psi \cdot v\right) .
$$


A.2. Identities of the second type. Applying $\bar{\partial}^{5} \partial_{t}$ to $9 \mathrm{~b}$ and computing the $L^{2}(\Omega)$ product with $\mu \bar{\partial}^{5} v^{i}$ we obtain

$$
\left(\mu \bar{\partial}^{5} v_{t}^{i}+\mu \bar{\partial}^{5} A_{i, t}^{k} q_{, k}+\mu A_{i}^{k} \bar{\partial}^{5} q_{, k t}, \bar{\partial}^{5} v^{i}\right)_{L^{2}}=\sum_{\substack{0<a+b<6 \\ a \leq 5, b \leq 1}} c_{a b}\left(\mu \bar{\partial}^{a} \partial_{t}^{b} A_{i}^{k} \bar{\partial}^{5-a} \partial_{t}^{1-b} q_{, k}, \bar{\partial}^{5} v^{i}\right)_{L^{2}},
$$

where $c_{a b}$ are constants appearing due to the usage of Leibniz product rule above. Recalling (47), we write

$$
\bar{\partial}^{5} A_{i, t}^{k}=-A_{i}^{s} \bar{\partial}^{5} \Psi_{, s t}^{r} A_{r}^{k}+\left\{\bar{\partial}^{5} \partial_{t}, A_{i}^{k}\right\},
$$

where $\left\{\bar{\partial}^{5} \partial_{t}, A_{i}^{k}\right\}$ stands for the lower order commutator defined in (47). With this identity, we obtain

$$
\begin{aligned}
& (\mu .17) \\
& \left(\mu \bar{\partial}^{5} A_{i, t}^{k} q_{, k}, \bar{\partial}^{5} v^{i}\right)_{L^{2}(\Omega)}=-\left(\mu A_{i}^{s} \bar{\partial}^{5} \Psi_{s t}^{r} A_{r}^{k} q_{, k}, \bar{\partial}^{5} v^{i}\right)_{L^{2}(\Omega)}+\left(\mu\left\{\bar{\partial}^{5} \partial_{t}, A_{i}^{k}\right\} q_{, k}, \bar{\partial}^{5} v^{i}\right)_{L^{2}(\Omega)} \\
& =-\int_{\Gamma} q_{, k} A_{i}^{s} \bar{\partial}^{5} \Psi_{t}^{r} A_{r}^{k} \bar{\partial}^{5} v^{i} N^{s}+\int_{\Omega} \mu A_{i}^{s} \bar{\partial}^{5} \Psi_{t}^{r} A_{r}^{k} q q_{, k} \bar{\partial}^{5} v_{, s}^{i}+\int_{\Omega} \mathscr{U}_{1} \\
& =-\int_{\Gamma} q_{, k} A_{i}^{s} \bar{\partial}^{5} \Psi_{t}^{r} A_{r}^{k} \bar{\partial}^{5} v^{i} N^{s}-\int_{\Omega} \mu A_{i}^{s} \bar{\partial}^{5} \Psi_{t}^{r} v^{r} \bar{\partial}^{5} v_{, s}^{i}+\int_{\Omega} \mathscr{U}_{1}
\end{aligned}
$$

where we have integrated-by-parts with respect to $x^{s}$ in the second equality and we have also used the identity $v^{r}=-A_{r}^{k} q_{, k}$ to write the last line more concisely. The error term $\mathscr{U}_{1}$ is given by

$$
\mathscr{U}_{1}=\left(\mu q_{, k} A_{i}^{s} A_{r}^{k}\right), s^{5} \bar{\partial}^{5} \Psi_{t}^{r} \bar{\partial}^{5} v^{i}+\mu\left\{\bar{\partial}^{5} \partial_{t}, A_{i}^{k}\right\} q_{, k}, \bar{\partial}^{5} v^{i}+\mu A_{i}^{s} \bar{\partial}^{5} \Psi_{t}^{r} A_{r}^{k} q_{, k}\left\{\bar{\partial}^{5}, \partial_{s}\right\} v^{i} .
$$

Furthermore, integrating by parts with respect to $x^{k}$

$$
\begin{aligned}
& \left(\mu A_{i}^{k} \bar{\partial}^{5} \partial_{t} q_{, k}, \bar{\partial}^{5} v^{i}\right)_{L^{2}}=\int_{\Omega} \mu A_{i}^{k} \partial_{k} \bar{\partial}^{5} \partial_{t} q \bar{\partial}^{5} v^{i}+\int_{\Omega} \mu A_{i}^{k}\left\{\bar{\partial}^{5}, \partial_{k}\right\} q_{t} \bar{\partial}^{5} v^{i} \\
& =-\int_{\Omega} \mu A_{i}^{k} \bar{\partial}^{5} q_{t} \bar{\partial}^{5} v_{, k}^{i}-\int_{\Omega}\left(\mu A_{i}^{k}\right)_{, k} \bar{\partial}^{5} q_{t} \bar{\partial}^{5} v^{i}+\int_{\Omega} \mu A_{i}^{k} \bar{\partial}^{5} q_{t}\left\{\bar{\partial}^{5}, \partial_{k}\right\} v^{i}+\int_{\Omega} \mu A_{i}^{k}\left\{\bar{\partial}^{5}, \partial_{k}\right\} q_{t} \bar{\partial}^{5} v^{i},
\end{aligned}
$$

where we have used $\bar{\partial}^{5} q_{t}=0$ on $\Gamma$. Summing (A.17) and (A.18), we obtain

$$
\begin{aligned}
& \left(\mu \bar{\partial}^{5} A_{i, t}^{k} q_{, k}+\mu A_{i}^{k} \bar{\partial}^{5} q_{, k t}, \bar{\partial}^{5} v^{i}\right)_{L^{2}(\Omega)}=-\int_{\Gamma} q_{, k} A_{i}^{s} \bar{\partial}^{5} \Psi_{t}^{r} A_{r}^{k} \bar{\partial}^{5} v^{i} N^{s}-\int_{\Omega} \mu A_{i}^{k} \bar{\partial}^{5} v_{, k}^{i}\left(\bar{\partial}^{5} q_{t}+\bar{\partial}^{5} \Psi_{t} \cdot v\right) \\
& +\int_{\Omega}\left(\mathscr{U}_{1}-\left(\mu A_{i}^{k}\right)_{, k} \bar{\partial}^{5} q_{t} \bar{\partial}^{5} v^{i}+\mu A_{i}^{k} \bar{\partial}^{5} q_{t}\left\{\bar{\partial}^{5}, \partial_{k}\right\} v^{i}+\mu A_{i}^{k}\left\{\bar{\partial}^{5}, \partial_{k}\right\} q_{t} \bar{\partial}^{5} v^{i}\right) .
\end{aligned}
$$

The first two terms on the right-hand side of A.19) will be the source of positive definite quadratic contributions to the energy. To extract the quadratic coercive contribution from the first integral on the right-hand side of A.19, note that $q_{, k}=N^{k} \partial_{N} q$ on $\Gamma$. Thus

$$
-\int_{\Gamma} q_{, k} A_{i}^{s} \bar{\partial}^{5} \Psi_{t}^{r} A_{r}^{k} \bar{\partial}^{5} v^{i} N^{s}=\int_{\Gamma}\left(-\partial_{N} q\right) \bar{\partial}^{5} \Psi_{t}^{r} \tilde{n}^{r} \bar{\partial}^{5} v^{i} \tilde{n}^{i}=\int_{\Gamma}\left(-\partial_{N} q\right) \overline{\partial^{5}} \Psi_{t} \cdot \tilde{n} \bar{\partial}^{5} v \cdot \tilde{n} .
$$

Just like in Section A.1 - as in the identities leading up to A.6 - we obtain

$$
\left(-\partial_{N} q\right) \bar{\partial}^{5} \Psi_{t} \cdot \tilde{n} \bar{\partial}^{5} v \cdot \tilde{n}=\left|\bar{\partial}^{5} h_{t}\right|^{2} R_{J}^{2}+\left|\sum_{a=0}^{4} c_{a}^{J} \bar{\partial}^{a} h_{t} \bar{\partial}^{5-a} N \cdot \tilde{n}\right|^{2}+2 \bar{\partial}^{5} h_{t} R_{J} \sum_{a=0}^{4} c_{a}^{J} \bar{\partial}^{a} h_{t} \bar{\partial}^{5-a} N \cdot \tilde{n}
$$

$$
+\sum_{l=1}^{4} a_{l}\left(-\partial_{N} q\right) \bar{\partial}^{5} \Psi \cdot \tilde{n} \bar{\partial}^{5} v \cdot \tilde{n} \bar{\partial}^{5-l}(v-w) \cdot \bar{\partial}^{l} \tilde{n}
$$


where $c_{a}^{J}=c_{a} J^{-1}$ and $c_{a}$ are some universal constants. As to the second term on the righthand side of A.19), note that

$$
A_{i}^{k} \bar{\partial}^{5} v_{, k}^{i}=\bar{\partial}^{5}\left(A_{i}^{k} v_{, k}^{i}\right)-\sum_{l=1}^{5} c_{l} \bar{\partial}^{l} A_{i}^{k} \bar{\partial}^{5-l} v_{, k}^{i}=-\bar{\partial}^{5}\left(q_{t}+v \cdot w\right)-\sum_{l=1}^{5} c_{l} \bar{\partial}^{l} A_{i}^{k} \bar{\partial}^{5-l} v_{, k}^{i},
$$

where $A_{i}^{k} v_{, k}^{i}=-\operatorname{div} \Psi v=-\left(q_{t}+v \cdot w\right)$ by the parabolic equation 9a). Thus

(A.21)

$$
\begin{aligned}
& -\int_{\Omega} \mu A_{i}^{k} \bar{\partial}^{5} v_{, k}^{i}\left(\bar{\partial}^{5} q_{t}+\bar{\partial}^{5} \Psi_{t} \cdot v\right) \\
& =\int_{\Omega} \mu \bar{\partial}^{5}\left(q_{t}+\Psi_{t} \cdot v\right)\left(\bar{\partial}^{5} q_{t}+\bar{\partial}^{5} \Psi_{t} \cdot v\right)+\sum_{l=1}^{5} c_{l} \int_{\Omega} \mu \bar{\partial}^{l} A_{i}^{k} \bar{\partial}^{5-l} v_{, k}^{i}\left(\bar{\partial}^{5} q_{t}+\bar{\partial}^{5} \Psi_{t} \cdot v\right) \\
& =\int_{\Omega} \mu\left(\bar{\partial}^{5} q_{t}+\bar{\partial}^{5} \Psi_{t} \cdot v\right)^{2}+\sum_{l=1}^{5} d_{l} \int_{\Omega} \mu \bar{\partial}^{5-l} \Psi_{t} \cdot \bar{\partial}^{l} v\left(\bar{\partial}^{5} q_{t}+\bar{\partial}^{5} \Psi_{t} \cdot v\right) \\
& \quad+\sum_{l=1}^{5} c_{l} \int_{\Omega} \mu \bar{\partial}^{l} A_{i}^{k} \bar{\partial}^{5-l} v_{, k}^{i}\left(\bar{\partial}^{5} q_{t}+\bar{\partial}^{5} \Psi_{t} \cdot v\right)
\end{aligned}
$$

Combining A.19) - A.21 we obtain

$$
\begin{aligned}
& \frac{1}{2} \frac{d}{d t} \int_{\Omega} \mu\left|\bar{\partial}^{5} v\right|^{2} d x+\int_{\Gamma}\left(-\partial_{N} q\right)\left|\bar{\partial}^{5} \Psi_{t} \cdot \tilde{n}\right|^{2} d x^{\prime}+\int_{\Omega} \mu\left(\bar{\partial}^{5} q_{t}+\bar{\partial}^{5} \Psi_{t} \cdot v\right)^{2} d x \\
& =\int_{\Gamma} \partial_{N} q\left|\sum_{a=0}^{4} c_{a}^{J} \bar{\partial}^{a} h_{t} \bar{\partial}^{5-a} \xi \cdot \tilde{n}\right|^{2}+\int_{\Omega} \mathscr{S}_{1}+\int_{\Gamma} \mathscr{H}_{1}
\end{aligned}
$$

with the error terms $\mathscr{S}_{j}$ and $\mathscr{H}_{j}$ given by:

$$
\begin{aligned}
\mathscr{S}_{j}= & \sum_{\substack{0<a+b<6-j \\
a \leq 7-2 j, b \leq j}} d_{a b} \mu \bar{\partial}^{a} \partial_{t}^{b} A_{i}^{k} \bar{\partial}^{7-2 j-a} \partial_{t}^{j-b} q_{, k} \bar{\partial}^{7-2 j} \partial_{t}^{j-1} v^{i}+\left(\mu q_{, k} A_{i}^{s} A_{r}^{k}\right)_{, s} \bar{\partial}^{7-2 j} \partial_{t}^{j} \Psi^{r} \bar{\partial}^{7-2 j} \partial_{t}^{j-1} v^{i} \\
& +\mu\left\{\bar{\partial}^{7-2 j} \partial_{t}^{j}, A_{i}^{k}\right\} q_{, k}, \bar{\partial}^{7-2 j} \partial_{t}^{j-1} v^{i}+\mu A_{i}^{s} \bar{\partial}^{7-2 j} \partial_{t}^{j} \Psi^{r} A_{r}^{k} q, k\left\{\bar{\partial}^{7-2 j}, \partial_{s}\right\} \partial_{t}^{j-1} v^{i} \\
& -\left(\mu A_{i}^{k}\right)_{, k} \bar{\partial}^{7-2 j} \partial_{t}^{j} q^{7} \bar{\partial}^{7-2 j} \partial_{t}^{j-1} v^{i}+\mu A_{i}^{k} \bar{\partial}^{7-2 j} \partial_{t}^{j} q\left\{\bar{\partial}^{7-2 j}, \partial_{k}\right\} \partial_{t}^{j-1} v^{i} \\
& +\mu A_{i}^{k}\left\{\bar{\partial}^{7-2 j}, \partial_{k}\right\} \partial_{t}^{j} q^{7-2 j} \partial_{t}^{j-1} v^{i}-\left(\bar{\partial}^{7-2 j} \partial_{t}^{j} q+\bar{\partial}^{7-2 j} \partial_{t}^{j} \Psi \cdot v\right)
\end{aligned}
$$

$$
\begin{aligned}
& \times \sum_{0 \leq a+b<6-j} d_{a b}\left(\mu \bar{\partial}^{a} \partial_{t}^{b} \Psi_{t} \cdot \bar{\partial}^{7-2 j-a} \partial_{t}^{j-1-b} v+\mu \bar{\partial}^{7-2 j-a} \partial_{t}^{j-1-b} A_{i}^{k} \bar{\partial}^{a} \partial_{t}^{b} v_{, k}^{i}\right) . \\
& \mathscr{H}_{j}=2 \partial_{N} q \bar{\partial}^{7-2 j} \partial_{t}^{j} h R_{J} \sum_{0 \leq a+b<7-j} c_{a b}^{J} \bar{\partial}^{a} \partial_{t}^{b} h \bar{\partial}^{7-2 j-a} \partial_{t}^{j-b} N \cdot \tilde{n} \\
& \quad+\left(-\partial_{N} q\right) \bar{\partial}^{7-2 j} \partial_{t}^{j} \Psi \cdot \tilde{n} \sum_{0 \leq a+b<6-j} d_{a b} \bar{\partial}^{7-2 j-a} \partial_{t}^{j-1-b}(v-w) \cdot \bar{\partial}^{a} \partial_{t}^{b} \tilde{n} .
\end{aligned}
$$

Note that the first line of A.24 appears as an expanded difference between two positive definite expressions $\left(-\partial_{N} q\right)\left|\bar{\partial}^{7-2 j} \partial_{t}^{j} \Psi \cdot \tilde{n}\right|^{2}$ and $\left(-\partial_{N} q\right)\left|\bar{\partial}^{7-2 j} \partial_{t}^{j} h\right|^{2}$. We do this just like after A.5 using the formula $\tilde{n}=J^{-1}\left(N+h N-h_{\theta} \tau\right)$ and the parametrization $\Psi(t, \xi)=$ $(1+h(t, \xi)) N$. Let now $\alpha=\left(\alpha_{1}, \alpha_{2}\right)$ be an arbitrary multi-index of order 5. Applying the operator $(1-\mu) \partial^{\alpha} \partial_{t}$ to $9 \mathrm{~b}$ ) and multiplying by $\partial^{\alpha} v^{i}$, we obtain

$$
\begin{aligned}
& \left((1-\mu) \partial^{\alpha} v_{t}^{i}+(1-\mu) \partial^{\alpha} A_{i, t}^{k} q_{, k}+(1-\mu) A_{i}^{k} \partial^{\alpha} q_{, k t}, \partial^{\alpha} v^{i}\right)_{L^{2}(\Omega)} \\
& =-\sum_{\substack{0<|\beta|+b<5 \\
\beta \leq \alpha ; b \leq 1}} c_{\beta b}\left((1-\mu) \partial^{\beta} \partial_{t}^{b} A_{i}^{k} \partial^{\alpha-\beta} \partial_{t}^{1-b} q_{, k}, \partial^{\alpha} v^{i}\right)_{L^{2}} .
\end{aligned}
$$


In the same way as above we arrive at the following energy identity

$$
\frac{1}{2} \frac{d}{d t} \int_{\Omega}(1-\mu)\left|\partial^{\alpha} v\right|^{2} d x+\int_{\Omega}(1-\mu)\left(\partial^{\alpha} q_{t}+\partial^{\alpha} \Psi_{t} \cdot v\right)^{2} d x=\int_{\Omega} \tilde{\mathscr{S}}_{1} d x
$$

where

(A.26)

$$
\begin{aligned}
& \tilde{\mathscr{S}}_{1}=\sum_{\substack{0<|\beta|+b<5 \\
\beta \leq \alpha ; b \leq 1}} c_{\beta b}(1-\mu) \partial^{\beta} \partial_{t}^{b} A_{i}^{k} \partial^{\alpha-\beta} \partial_{t}^{1-b} q_{, k} \partial^{\alpha} v^{i}+(1-\mu)\left(\left(q_{, k} A_{i}^{s} A_{r}^{k}\right), \partial^{\alpha} \Psi_{t}^{r} \partial^{\alpha} v^{i}+\left\{\partial^{\alpha} \partial_{t}, A_{i}^{k}\right\} q_{, k} \partial^{\alpha} v^{i}\right) \\
& -(1-\mu) \sum_{0<\beta \leq \alpha} c_{\beta} \mu \partial^{\alpha-\beta} \Psi_{t} \cdot \bar{\partial}^{l} v\left(\bar{\partial}^{\alpha} q_{t}+\bar{\partial}^{\alpha} \Psi_{t} \cdot v\right)-(1-\mu) \sum_{\beta \leq \alpha} c_{l} \mu \partial^{\beta} A_{i}^{k} \bar{\partial}^{\alpha-\beta} v_{, k}^{i}\left(\bar{\partial}^{\alpha} q_{t}+\bar{\partial}^{\alpha} \Psi_{t} \cdot v\right),
\end{aligned}
$$

For a general $j \in\{1,2,3\}$ we have

$$
\begin{aligned}
& \tilde{\mathscr{S}}_{j}=\sum_{\substack{0<|\beta|+b<7-2 j \\
\beta \leq \alpha ; b \leq j}} d_{\beta b}(1-\mu) \partial^{\beta} \partial_{t}^{b} A_{i}^{k} \partial^{\alpha-\beta} \partial_{t}^{j-b} q_{, k} \partial^{\alpha} v^{i}+(1-\mu) \tilde{\mathscr{U}}_{1} \\
& -(1-\mu) \sum_{0 \leq|\beta|+b<|\alpha|} d_{\beta b} \mu \partial^{\beta} \partial_{t}^{b} \Psi_{t} \cdot \partial^{\alpha-\beta} \partial_{t}^{j-1-b} v\left(\bar{\partial}^{\alpha} \partial_{t}^{j} q+\bar{\partial}^{\alpha} \partial_{t}^{j} \Psi \cdot v\right) \\
& -(1-\mu) \sum_{0 \leq|\beta|+b<|\alpha|} d_{\beta b} \mu \partial^{\alpha-\beta} \partial_{t}^{j-1-b} A_{i}^{k} \bar{\partial}^{\beta} \partial_{t}^{b} v_{, k}^{i}\left(\bar{\partial}^{\alpha} \partial_{t}^{j} q+\bar{\partial}^{\alpha} \partial_{t}^{j} \Psi \cdot v\right) .
\end{aligned}
$$

Summing the identities A.22 and A.25 we arrive at

$$
\begin{aligned}
& \frac{1}{2} \frac{d}{d t}\left\{\int_{\Omega} \mu\left|\bar{\partial}^{7-2 j} \partial_{t}^{j} v\right|^{2}+\sum_{|\alpha|=7-2 j} \int_{\Omega}(1-\mu)\left|\partial^{\alpha} \partial_{t}^{j} v\right|^{2}\right\}+\int_{\Gamma}\left(-\partial_{N} q\right) R_{J}^{2}\left|\bar{\partial}^{7-2 j} \partial_{t}^{j} h\right|^{2} \\
& +\int_{\Omega} \mu\left(\bar{\partial}^{7-2 j} \partial_{t}^{j} q+\bar{\partial}^{7-2 j} \partial_{t}^{j} \Psi \cdot v\right)^{2}+\sum_{|\alpha|=7-2 j} \int_{\Omega}(1-\mu)\left(\partial^{\alpha} \partial_{t}^{j} q+\partial^{\alpha} \partial_{t}^{j} \Psi \cdot v\right)^{2} \\
& =\int_{\Omega}\left(\mathscr{S}_{j}+\tilde{\mathscr{S}}_{j}\right)+\int_{\Gamma} \mathscr{H}_{j}, \quad j=1,2,3 .
\end{aligned}
$$

Summing the identities A.13) for $j=0,1,2,3$ and A.28 for $j=1,2,3$, we conclude the proof of Proposition 3.1

\section{Appendix B. Proof of the inequality 75}

We use the comparison function $P$ defined in 98 with the same $\kappa_{2}$ and $\kappa_{1}$. Note that $\kappa_{1}=C_{*} \varepsilon c_{1}$ is defined as a multiple of $c_{1}$ for some constant $C_{*}>0$. Using (100) and upon possibly enlarging $C_{*}$, we infer $\left(\partial_{t}-a_{i j}-b_{i}\right)\left(-q_{t}+P\right) \leq 0$. Theorem 1 from [41] guarantees

$$
-q_{t}+P \leq C_{0} c_{1} \rho e^{\left(-\lambda_{1}+C \varepsilon\right) t},
$$

where $\rho(r)=1-r$ stands for the distance function to the boundary $\Gamma$. Note that the constant $\kappa_{1}$ in the definition (98) is chosen right after 102). It is in particular proportional to $E_{\beta}(0)^{1 / 2} \leq\left\|q_{0}\right\|_{4}$. By definition of $K$ we have that $\left\|q_{0}\right\|_{4} \leq K\left\|q_{0}\right\|_{0}$. Since however $\left\|q_{0}\right\|_{0} \leq K c_{1}$, we obtain $P / \rho \leq C K^{2} c_{1} e^{-3 \lambda_{1} t / 2}$. Similarly, the constant $C_{0}$ is proportional to the $L^{\infty}$-norm of the initial datum for $-q_{t}+P$, wherefrom we again obtain $C_{0} \leq K^{2} c_{1}$ by the same argument as above. Dividing by $\rho$ in $(\bar{B} .1)$, from the above inequality, we infer that

$$
\left|\partial_{N} q_{t}\right|_{\infty} \leq C K^{2} c_{1} e^{\left(-\lambda_{1}+C \varepsilon\right) t} .
$$

This proves the inequality 75 . 


\section{ACKNOWLEDGEMENTS}

MH was supported by the National Science Foundation under grant NSF DMS-1211517 and SS was supported by the National Science Foundation under grant DMS-1001850. The authors would like to thank David Jerison for discussions leading to Lemma2.1. They also thank the anonymous referee for helpful comments.

\section{REFERENCES}

[1] Adams, R. A. Sobolev Spaces. 1978 Academic Press,

[2] Almgren, F., Wang, L. Mathematical existence of crystal growth with Gibbs-Thomson curvature effects. $J$. Geom. Anal. 10 (2000), no.1, 1-100.

[3] Athanasopoulos, I., Caffarelli, L. A., Salsa, S. Regularity of the free boundary in parabolic phase-transition problems. Acta Math., 176 (1996), 245-282.

[4] Athanasopoulos, I., Caffarelli, L. A., Salsa, S. Phase transition problems of parabolic type: flat free boundaries are smooth. Comm. Pure Appl. Math. 51 (1998), 77-112.

[5] Caffarelli, L. A. The regularity of free boundaries in higher dimensions. Acta Math. 139 (1977), no. 3-4, $155-184$.

[6] Caffarelli, L. A. Some aspects of the one-phase Stefan problem. Indiana Univ. Math. J. 27 (1978), 73-77.

[7] Caffarelli, L. A., Friedman, A. Continuity of the temperature in the Stefan problem. Indiana Univ. Math. J. 28 (1979), no. 1, 53-70.

[8] Caffarelli, L. A., Evans, L.C. Continuity of the temperature in the two-phase Stefan problem. Arch. Rational Mech. Anal. 81 (1983), 199-220.

[9] Caffarelli, L. A., Salsa, S. A Geometric Approach to Free Boundary Problems. American Mathematical Society, Providence, RI, 2005.

[10] Castro, A., Córdoba, D., Gancedo, F. Some recent results on the Muskat problem. Journees Equations aux Derivees Partielles (2010), Exp. No. 5.

[11] Choi, S., Kim, I. Regularity of one-phase Stefan problem near Lipschitz initial data, Amer. J. Math. 132 (2010), no. 6.

[12] Constantin, P., Córdoba, D., Gancedo, F., Strain, R. M. On the global existence for the Muskat problem. $J$. Eur. Math. Soc. 15 (2013), 201-227.

[13] Córdoba, A., Córdoba, D., Gancedo, F. Interface evolution: the Hele-Shaw and Muskat problems. Annals of Math. 173 (2011), no. 1, 477-542.

[14] Córdoba, A., Córdoba, D., Gancedo, F. Porous media: the Muskat problem in 3D. To appear in Analysis \& $P D E$.

[15] Coutand, D., Shkoller, S. Well-posedness of the free-surface incompressible Euler equations with or without surface tension. J. Amer. Math. Soc., 20 (2007), no. 3, 829-930.

[16] Coutand, D., Shkoller, S. A simple proof of well-posedness for the free surface incompressible Euler equations. Discr. Cont. Dyn. Systems, Series S 3 (2010), no. 3, 429-449.

[17] Coutand, D., Hole, J., Shkoller, S. Well-posedness of the free-boundary compressible 3-D Euler equations with surface tension and the zero surface tension limit. Available online at http://arxiv.org/abs/1208.2726

[18] Coutand, D., Shkoller, S. Well-posedness in smooth function spaces for the moving-boundary 3-D compressible Euler equations in physical vacuum. Arch. Rational Mech. Anal. 206 (2012), 515-616.

[19] Daskalopoulos, P., Lee, K. All time smooth solutions of the one-phase Stefan problem and the Hele-Shaw flow. Comm. Partial Differential Equations 29 (2004), no. 1-2, 71-89.

[20] Escher, J., Prüss, J., Simonett, G. Analytic solutions for a Stefan problem with Gibbs-Thomson correction. J. Reine Angew. Math. 563 (2003), 1-52.

[21] Friedman, A. Partial Differential Equations of Parabolic Type. Prentice-Hall, Englewood Cliffs, N.J., 1964.

[22] Friedman, A. The Stefan problem in several space variables. Trans. Amer. Math. Soc. 133 (1968), 51-87.

[23] Friedman, A. Variational Principles and Free Boundary Problems. Wiley, New York, 1982.

[24] Friedman, A., Kinderlehrer, D. A one phase Stefan problem. Indiana Univ. Math. J. 25 (1975), 1005-1035.

[25] Friedman, A., Reitich, F. The Stefan problem with small surface tension. Trans. Amer. Math. Soc. 328 (1991), 465-515.

[26] Frolova, E. V., Solonnikov, V.A. $L_{p}$-theory for the Stefan problem. J. Math. Sci. 99 (2000), no. 1, 989-1006.

[27] Hadžić, M. Orthogonality conditions and asymptotic stability in the Stefan problem with surface tension. Arch. Rational Mech. Anal. 203 (2012), no. 3, 719-745.

[28] Hadžić, M., Guo, Y. Stability in the Stefan problem with surface tension (I). Commun. Partial Differential Eqns., 35 (2010), no. 2, 201-244. 
[29] Hadžić, M., Shkoller, S. Well-posedness for the classical Stefan problem and the vanishing surface tension limit. Submitted, available online at http://arxiv.org/abs/1112.5817

[30] Hanzawa, E.I. Classical solution of the Stefan problem. Tohoku Math, J. 33 (1981), 297-335.

[31] Jerison, D., Levine, L., Sheffield, S. Logarithmic fluctuations for internal DLA. J. Amer. Math. Soc. 25 (2012), 271-301.

[32] Kamenomostskaya, S. L. On Stefan problem. Mat. Sb. 53 (1961), 489-514.

[33] Kim, I. Uniqueness and Existence of Hele-Shaw and Stefan problem. Arch. Rat. Mech. Anal. 168 (2003), 299-328.

[34] Kim, I., Požar, N. Viscosity solutions for the two-phase Stefan problem. Comm. PDE. 36 (2011), no.1, $42-66$.

[35] Kinderlehrer, D., Nirenberg, L. Regularity in free boundary problems. Ann. Scuola Norm. Sup. Pisa Cl. Sci 4 (1977), no. 2, 373-391.

[36] Kinderlehrer, D., Nirenberg, L. The smoothness of the free boundary in the one-phase Stefan problem. Comm. Pure Appl. Math. 31 (1978), no. 3, 257-282.

[37] KосH, H. Classical solutions to phase transition problems are smooth. Comm. Partial Differential Equations 23 (1998), no. 3-4, 389-437.

[38] Ladyženskaja, O. A., Solonnikov V. A., Uralceva, N.N. Linear and Quasilinear equations of Parabolic Type. Trans. Math. Monographs Amer. Math. Soc., Providence, RI, 23, 1968.

[39] Luckhaus, S. Solutions for the two-phase Stefan problem with the Gibbs-Thomson law for the melting temperature. Europ. J. Appl. Math. 1 (1990), 101-111.

[40] Meirmanov, A. M. The Stefan Problem. De Gruyter Expositions in Mathematics, 3, 1992.

[41] Oddson, J. K. On the rate of decay of solutions to parabolic differential equations. Pacific Journal of Mathematics 29 (1969), no. 2, 389-396.

[42] Prüss, J., Saal, J., Simonett, G. Existence of analytic solutions for the classical Stefan problem. Math. Ann. 338 (2007), 703-755.

[43] Prüss, J., Simonett, G., Zacher, R. Qualitative behavior of solutions for thermodynamically consistent Stefan problems with surface tension. Arch. Ration. Mech. Anal. 207 (2013), 611- 667.

[44] Quirós, F., Vázquez, J. L. Asymptotic convergence of the Stefan problem to Hele-Shaw Trans. Am. Math. Soc. 353 (2000), no. 2, 609-634.

[45] Radkevich, E.V. Gibbs-Thomson law and existence of the classical solution of the modified Stefan problem. Soviet Dokl. Acad. Sci. 316 (1991), 1311-1315.

[46] Lord Rayleigh. On the instability of jets. Proc. London Math. Soc. 1 (1878), s1-10, 4-13.

[47] Röger, M. Solutions for the Stefan problem with Gibbs-Thomson law by a local minimisation. Interfaces Free Bound. 6 (2004), 105-133.

[48] Taylor, G. The Instability of Liquid Surfaces when Accelerated in a Direction Perpendicular to their Planes. I Proc. R. Soc. Lond. A 201 (1950), no. 1065, 192-196.

[49] Visintin, A. Introduction to Stefan-Type Problems. Handbook of Differential Equations, Evolutionary equations, Elsevier B.V., North-Holland, 4, 377-484, Elsevier B.V., North-Holland, 2008

[50] $\mathrm{Wu}, \mathrm{S}$. Well-posedness in Sobolev spaces of the full water wave problem in 2-D. Invent. Math. 130 (1997), no. $1,39-72$. 